University of Louisville

ThinkIR: The University of Louisville's Institutional Repository

Electronic Theses and Dissertations

$12-2017$

\title{
Nonspreading solutions in integro-difference models with allee and overcompensation effects.
}

Garrett Luther Otto

University of Louisville

Follow this and additional works at: https://ir.library.louisville.edu/etd

Part of the Dynamic Systems Commons, and the Population Biology Commons

\section{Recommended Citation}

Otto, Garrett Luther, "Nonspreading solutions in integro-difference models with allee and overcompensation effects." (2017). Electronic Theses and Dissertations. Paper 2858.

https://doi.org/10.18297/etd/2858

This Doctoral Dissertation is brought to you for free and open access by ThinkIR: The University of Louisville's Institutional Repository. It has been accepted for inclusion in Electronic Theses and Dissertations by an authorized administrator of ThinkIR: The University of Louisville's Institutional Repository. This title appears here courtesy of the author, who has retained all other copyrights. For more information, please contact thinkir@louisville.edu. 


\title{
NONSPREADING SOLUTIONS IN INTEGRO-DIFFERENCE MODELS WITH ALLEE AND OVERCOMPENSATION EFFECTS
}

\author{
By \\ Garrett Luther Otto \\ B.S. University of Kentucky, 2001

\begin{abstract}
A Dissertation
Submitted to the Faculty of the

College of Arts and Sciences of the University of Louisville

in Partial Fulfillment of the Requirements

for the Degree of
\end{abstract} \\ Doctor of Philosophy \\ in \\ Applied and Industrial Mathematics \\ Department of Mathematics \\ University of Louisville \\ Louisville, KY
}

December 2017 



\title{
NONSPREADING SOLUTIONS IN INTEGRO-DIFFERENCE MODELS WITH ALLEE AND OVERCOMPENSATION EFFECTS
}

\author{
Submitted by \\ Garrett Luther Otto \\ A Dissertation Approved on \\ Nov. 17, 2017 \\ by the Following Dissertation Committee: \\ Dr. Bingtuan Li, \\ Dissertation Director \\ Dr. Sarah Emery \\ Dr. Gung-Min Gie \\ Dr. Changbing Hu
}

Dr. David Swanson 


\section{DEDICATION}

Dedicated to my wife, Jin Shang, whose patience, support, and love have made it possible for me complete my graduate studies. I would also like to dedicate this to my son, Carter, who is always an inspiration. 


\section{ACKNOWLEDGEMENTS}

I would like to thank the following people for their invaluable contributions:

- my parents for teaching me to value education and reason

- my wife, Jin Shang, for bringing light and warmth to my life, as well as a large quantity of practical advice

- Dr. Bingtuan Li for his mentorship in mathematical biology

- the thesis committee for their valuable feedback

- Dr. Ryan Gill, Trevor Leach, and Dr. Thomas Riedel for their assistance with LATEX typesetting issues

- Finally I would also like to thank Dr. Ryan Gill, Dr. Robert Powers, and Dr.Thomas Riedel, for all their assistance with the various administrative, academic, or teaching related issues that have arisen throughout my stay at $\mathrm{U}$ of $\mathrm{L}$. 


\section{ABSTRACT \\ NONSPREADING SOLUTIONS IN INTEGRO-DIFFERENCE MODELS WITH ALLEE AND OVERCOMPENSATION EFFECTS}

Garrett Luther Otto

November 17,2017

Previous work in Integro-Difference models have generally considered Allee effects and over-compensation separately, and have either focused on bounded domain problems or asymptotic spreading results. Some recent results by Sullivan et al. (2017 PNAS 114(19), 5053-5058) combining Allee and over-compensation in an Integro-Difference framework have shown chaotic fluctuating spreading speeds. In this thesis, using a tractable parameterized growth function, we analytically demonstrate that when Allee and over-compensation are present solutions which persist but essentially remain in a compact domain exist. We investigate the stability of these solutions numerically. We also numerically demonstrate the existence of such solutions for more general growth functions. 
TABLE OF CONTENTS

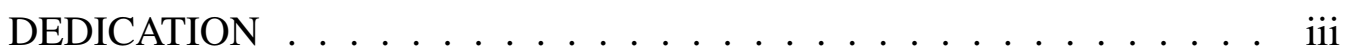

ACKNOWLEDGEMENTS ................. iv

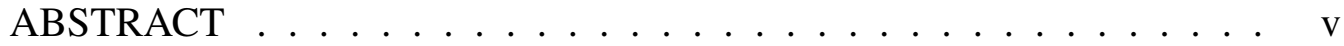

LIST OF FIGURES $\ldots \ldots \ldots \ldots$. . . . . . . . . . . viii

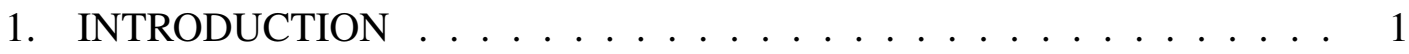

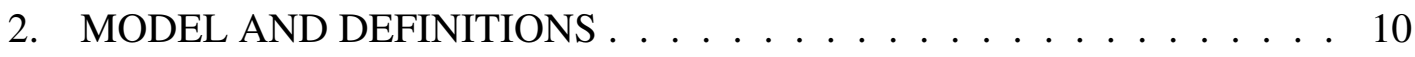

3. EQUILIBRIUM SOLUTIONS-ANALYTICAL RESULTS . . . . . . . . . 12

3.0.1 Uniform dispersal kernel . . . . . . . . . . . . . . . 16

3.0.2 Gaussian dispersal kernel _ . . . . . . . . . . 22

3.0.3 Exponential power distribution $\ldots \ldots \ldots$. . . . . 27

4. NUMERICAL RESULTS . . . . . . . . . . . . . . . . 38

4.0.4 Uniform dispersal with piecewise constant fecundity func-

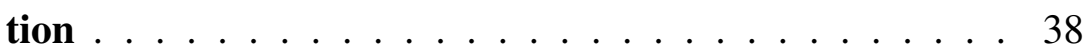

4.0.4.1 Perturbed equilibria $\ldots \ldots \ldots$

4.0.4.2 Initial domain size $\ldots \ldots$. . . . . . . . . . . . 45

4.0.5 Gaussian dispersal with piecewise constant fecundity

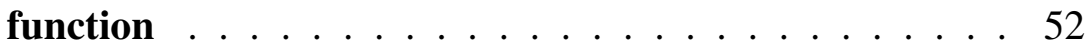

4.0.6 Leptokurtic dispersal kernel with piecewise constant fecundity function . . . . . . . . . . . . . . 57

4.0.7 Numerical results for other growth functions . . . . . . 62

4.0.7.1 Truncated Ricker function . . . . . . . . . . . . . 62

4.0.7.2 Hill-Ricker function . . . . . . . . . . . . . 65 
4.0.7.3 Truncated-Ricker function with linear strong-Allee

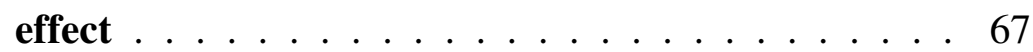

4.0 .8 Patch Formation . . . . . . . . . . . 70

4.0.8.1 Two interacting stable equilibrium $\ldots . . . . .70$

4.0.8.2 Two interacting period-2 equilibrium . . . . . . . 73

4.0.8.3 Patch formation over large perturbed initial domain with single stable equilibria $\ldots \ldots$. . . . . . 81

4.0.8.4 Patch formation over large perturbed initial domain with period-2 equilibria $\ldots \ldots$. . . . . . . 84

4.0.9 Spread with essential extinction . . . . . . . . . . 88

5. REMARKS AND CONCLUSION . . . . . . . . . . . . . . . 106

REFERENCES . . . . . . . . . . . . . . . . . . . 109

CURRICULUM VITAE . . . . . . . . . . . . . . . . . 115 


\section{LIST OF FIGURES}

Figure 3.1. fecundity function and the line $\mathrm{y}=\mathrm{u} \ldots \ldots \ldots$

Figure 3.2. plot of $G_{\{a, b\}}(x) \ldots \ldots \ldots \ldots \ldots \ldots$

Figure 3.3. Region $\mathscr{A}$ of the $(a, b)$ plane. . . . . . . . . . . 17

Figure 3.4. Regions of the $(a, b)$ plane where the Boolean vector $\{a+b>$ $\left.\frac{1}{2}, 2 b>\frac{1}{2}, 2 a>\frac{1}{2}, b-a>\frac{1}{2}, b>\frac{1}{2}, a>\frac{1}{2}\right\}$ takes distinct values. . 18

Figure 3.5. Constant $n_{2}$ slices of parameter space. The shaded regions are parameters values where an equilibrium solution exists. . . . . . . 21

Figure 3.6. The equilibrium solution with parameter values $\left(n_{0}, n_{1}, n_{2}\right)=(1.3,1.5,2.3) 22$

Figure 3.7. Sub-Figure (a) illustrates a set of parameters having no solution to the algebraic system in Theorem 3.1, and thus no equilibria. SubFigure (b) illustrates a set of parameters having a single solution, and thus a unique equilibrium. In sub-figure (c) we see a set of parameters where there are two distinct equilibria. . . . . . . 24

Figure 3.8. Constant $n_{2}$ slices of parameter space. The salmon colored regions are parameter values with a single equilibria. The blue regions are parameter values with 2 distinct equilibria. . . . . . . . . 26

Figure 3.9. Typical equilibrium solutions for the Gaussian dispersal kernel. In sub-figure (a) the parameters have a unique equilibria. In sub-figure (b) the parameters have two distinct equilibria. . . . . . . . . . 27

Figure 3.10. A plot of the kurtosis vs. $\gamma$ and probability density functions for several values of $\gamma$. The dashed line $y=1.8$ in sub-figure (a) is the kurtosis of the uniform distribution. . . . . . . . . . . . . 29 
Figure 3.11. Plot of the parameter regions where equilibriums exist when $k$ is an exponential power distribution with $\gamma=1.2$. Note differing scales in sub-plot $(a)$ and $(b) . \ldots \ldots . \ldots . \ldots . \ldots 31$

Figure 3.12. Plot of the parameter regions where equilibriums exist when $k$ is an exponential power distribution with $\gamma=1.5$. Note differing scales in sub-plot (a) and (b). . . . . . . . . . . . . . 32

Figure 3.13. Plot of the parameter regions where equilibriums exist when $k$ is an exponential power distribution with $\gamma=3$. Note differing scales in sub-plot (a) and (b). . . . . . . . . . . . . . . 33

Figure 3.14. Plot of the parameter regions where equilibriums exist when $k$ is an exponential power distribution with $\gamma=5$. Note differing scales in sub-plot (a) and (b). . . . . . . . . . . . . . 34

Figure 3.15. A comparison of the parameter values producing equilibrium for $\gamma=1.2$ to $\gamma=1.5$. Note the region for $\gamma=1.2$ is a proper subset of that for $\gamma=1.5$ for both values of $n_{2}$ depicted. . . . . . . . . 35

Figure 3.16. A comparison of the parameter values producing equilibrium for $\gamma=1.5$ to $\gamma=3$. Note the region for $\gamma=1.5$ is a proper subset of that for $\gamma=3$ for both values of $n_{2}$ depicted. . . . . . . . . 36

Figure 3.17. A comparison of the parameter values producing equilibrium for $\gamma=3$ to $\gamma=5$. Note the region for $\gamma=3$ is a proper subset of that for $\gamma=5$ for both values of $n_{2}$ depicted. . . . . . . . . . 37

Figure 4.1. A domain-size vs. time plot of an equilibrium where perturbation leads to extinction. $\left\{n_{0}, n_{1}, n_{2}\right\}=\{1.1,1.4,2.5\}$. The equilibrium is $k * G_{\{0.2334,0.5548\}}$, and the perturbed initial state is $k *$ $\widetilde{G}_{\{-0.5553,-0.2212,0.2353,0.5586\}} \ldots \ldots \ldots \ldots$. . . . . . 40 
Figure 4.2. A case where perturbations lead to spreading solutions. $\left\{n_{0}, n_{1}, n_{2}\right\}=$ $\{1.4,1.6,2.5\}$. The equilibrium is $k * G_{\{0.1508,0.4236\}}$, and the perturbed initial state is $k * \widetilde{G}_{\{-0.4189,-0.1439,0.1471,0.4193\}} \ldots \ldots \ldots 41$

Figure 4.3. A domain-size vs. time plot for a perturbation leading to a period-5 orbit. $\left\{n_{0}, n_{1}, n_{2}\right\}=\{1.3,1.45,2.5\}$. The equilibrium is $k * G_{\{0.2205,0.5122\}}$, and the perturbed initial state is $k * \widetilde{G}_{\{-0.5024,-0.2078,0.2134,0.5035\}}$. . 42

Figure 4.4. A plot of $u_{30}(x)$ through $u_{35}(x)$ showing a complete period. The parameters and initial condition are the same as in Figure 4.3 . . . . 43

Figure 4.5. A case where the same parameters used in Figure 4.4 have a different orbit when a different initial condition is used. The periodicity of this orbit is 105 . The initial state is $k * \widetilde{G}_{\{-0.5160,-0.2232,0.2387,0.5193\}}$. 44

Figure 4.6. Domain size after 88 iterations (red), 100 iterations (green), and 112 iterations (blue) as a function of initial domain half-width. In sub-figure (b) a the domain vs. time plot is shown for $w=0.5$. In sub-figure (c) the domain vs time plot is shown for $w=0.75$. Parameter values used are $\left(n_{0}, n_{1}, n_{2}\right)=(1.6,1.75,2.1) \ldots \ldots 46$

Figure 4.7. Domain size after 88 iterations (red), 100 iterations (green), and 112 iterations (blue) as a function of initial domain half-width. In sub-figure (b) a the domain vs. time plot is shown for $w=0.6$. In sub-figure (c) the domain vs time plot is shown for $w=0.665$. Parameter values used are $\left(n_{0}, n_{1}, n_{2}\right)=(1.2,1.9,2.1) \ldots \ldots .47$

Figure 4.8. In sub-figure (a) domain size after 88 iterations (red), 100 iterations (green), and 112 iterations (blue) as a function of initial domain half-width. In sub-figure (b) domain size vs. $t$ for $w=0.6$ is depicted. Parameter values used are $\left(n_{0}, n_{1}, n_{2}\right)=(1.2,1.6,2.1) \ldots .48$ 
Figure 4.9. Domain size after 88 iterations (red), 100 iterations (green), and 112 iterations (blue) as a function of initial domain half-width. In sub-figure (b) a the domain vs. time plot is shown for $w=0.62$. In sub-figure (c) the domain vs time plot is shown for $w=0.475$. Parameter values used are $\left(n_{0}, n_{1}, n_{2}\right)=(1.3,1.6,2.5) \ldots \ldots 49$

Figure 4.10. Domain size after 88 iterations (red), 100 iterations (green), and 112 iterations (blue) as a function of initial domain half-width. In sub-figure (b) a the domain vs. time plot is shown for $w=0.86$. In sub-figure (c) the domain vs time plot is shown for $w=0.82$. Parameter values used are $\left(n_{0}, n_{1}, n_{2}\right)=(1,1.7,2.5) \ldots \ldots 50$

Figure 4.11. In sub-figure (a) domain size after 88 iterations (red), 100 iterations (green), and 112 iterations (blue) are depicted as a function of initial domain half-width. In sub-figure (b) the domain size vs. time is depicted for a $w$ with a non-spreading solution, in sub-figure (c) the domain size vs. time is depicted for a $w$ producing extinction. Parameter values used are $\left(n_{0}, n_{1}, n_{2}\right)=(1.1,1.4,2.5) \ldots \ldots 51$

Figure 4.12. A case where perturbations lead to period-2 oscillations. $\left\{n_{0}, n_{1}, n_{2}\right\}=$ $\{1.25,1.36,2.6\}$. The equilibriums are $k * G_{\{0.1399,0.3322\}}$ and $k *$ $G_{\{0.3087,0.5279\}}$. The perturbed initial state used in this figure is $k * \widetilde{G}_{\{-0.3373,-0.1560,0.1374,0.3330\}} \ldots \ldots \ldots \ldots$. . . . . . 54

Figure 4.13. A case where perturbations lead to period-4 oscillations. $\left\{n_{0}, n_{1}, n_{2}\right\}=$ $\{1.25,1.3,2.6\}$. The equilibrium is $k * G_{\{0.0849,0.2618\}}$. The perturbed initial state used in the figure is $k * \widetilde{G}_{\{-0.2634,-0.0933,0.0845,0.2618\}} \cdot 55$

Figure 4.14. A case where perturbations lead to irregular oscillations. $\left\{n_{0}, n_{1}, n_{2}\right\}=$ $\{1.05,1.34,2.6\}$. The equilibriums are $k * G_{\{0.0929,0.2889\}}$ and $k *$ $G_{\{0.3091,0.5576\}}$. The perturbed initial state used in this figure is $k * \widetilde{G}_{\{-0.2866,-0.0884,0.1011,0.2891\}} \ldots \ldots \ldots \ldots$ 
Figure 4.15. Perturbations leading to a period-2 oscillations. $\left\{n_{0}, n_{1}, n_{2}\right\}=\{1.53,1.63,2.1\}$. The equilibria is $k * G_{\{0.0830,0.4149\}}$. The perturbed initial state used in this figure is $k * \widetilde{G}_{\{-0.4124,-0.0821,0.0786,0.4122\}} \ldots \ldots . \ldots 58$

Figure 4.16. Perturbations lead to a stable equilibrium. $\left\{n_{0}, n_{1}, n_{2}\right\}=\{1.1,1.55,2.1\}$. The equilibria is $k * G_{\{0.0092,0.3181\}}$. The perturbed initial state used in this figure is $k * \widetilde{G}_{\{-0.3181,-0.0097,0.0069,0.3180\}} \ldots \ldots$. . . . . 59

Figure 4.17. Perturbations leading to period-4 oscillations. $\left\{n_{0}, n_{1}, n_{2}\right\}=\{1.24,1.37,2.5\}$. The equilibria is $k * G_{\{0.0977,0.2904\}}$. The perturbed initial state used in this figure is $k * \widetilde{G}_{\{-0.2943,-0.1041,0.0985,0.2933\}} \ldots \ldots$. . . . . 60

Figure 4.18. Perturbations leading to period-6 oscillations. $\left\{n_{0}, n_{1}, n_{2}\right\}=\{1.1,1.38,2.5\}$. The equilibriums are $k * G_{\{0.0973,0.3028\}}$. The perturbed initial state used in this figure is $k * \widetilde{G}_{\{-0.2991,-0.0982,0.0938,0.2983\}} \ldots \ldots$. . . . 61

Figure 4.19. Plots of the growth function, transient iterations, and asymptotic attractor for the truncated Ricker function with $r=1.2$ and $q=1.7 . \quad 64$

Figure 4.20. The Hill-Ricker growth function $g(u)=u \exp (2.5(1-u))\left(\frac{\left(1+1.3^{7}\right)\left(\frac{u}{1.3}\right)^{7}}{1+\left(\frac{u}{1.3}\right)^{7}}\right) \cdot 65$

Figure 4.21. Plots of solution curves for $r=2.5 \ldots \ldots \ldots 6$

Figure 4.22. Plots of solution curves for $r=2.8 \ldots \ldots$. . . . . . . . . 67

Figure 4.23. Plots of the growth function, transient iterations, and asymptotic attractor for the growth function in Eq. 4.3 with $\rho=0.4$. . . . . 69

Figure 4.24. Total population of end-state versus spacing parameter $(w)$. . . . 71

Figure 4.25. Evolution of solution ending a merger of patches. $w=1.77 \ldots 72$

Figure 4.26. Evolution of solution ending in extinction. $w=1.65 \ldots \ldots 73$

Figure 4.27. Total population of even year end-state versus spacing parameter (w) for symmetric initial data. Grey dashed lines are multiples of 2.2. 75

Figure 4.28. Illustration of collapse from 2-patches to one patch for symmetric initial data. $w=1.387 \ldots \ldots \ldots \ldots \ldots$ 
Figure 4.29. Illustration of extinction from symmetric two-patch initial data. $w=$

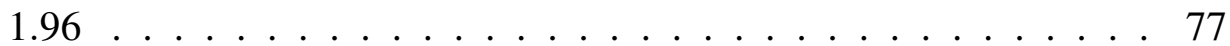

Figure 4.30. Total population of final state odd year end-state versus spacing parameter $(w)$ for asymmetric initial data. . . . . . . . . . 78

Figure 4.31. Illustration of merger from 2-patches to one patch for asymmetric initial data. $w=1.47 \ldots \ldots \ldots \ldots \ldots$

Figure 4.32. Illustration of out of phase 2 patch initial data, forming into an inphase 2 patch oscillation. $w=1.646 \ldots \ldots$. . . . . . . 80

Figure 4.33. Illustration of extinction from asymmetric two-patch initial data. $w=1.3 \ldots \ldots \ldots \ldots \ldots \ldots$

Figure 4.34. Pattern formation with $\sigma=6$. Initial data is shown in blue, iterations $110-120$ are shown in red. . . . . . . . . . . 83

Figure 4.35. Pattern formation with $\sigma=2$. Initial data is shown in blue, iterations $110-120$ are shown in red. . . . . . . . . . . . 83

Figure 4.36. Pattern formation with $\sigma=0.25$. Initial data is shown in blue, iterations $110-120$ are shown in red. . . . . . . . . . . . . 84

Figure 4.37. Pattern formation with $\sigma=6$. Sub-figure (a) depicts transient phenomena, while sub-figure (b) shows the terminal state. In (b) oddyears are depicted in blue, and even-years are red. . . . . . . . . 85

Figure 4.38. Pattern formation with $\sigma=2$. Sub-figure (a) depicts transient phenomena, while sub-figure (b) shows the terminal state. In (b) oddyears are depicted in blue, and even-years are red. . . . . . . . . . 86

Figure 4.39. Pattern formation with $\sigma=2$. Sub-figure (a) depicts transient phenomena, while sub-figure (b) shows the terminal state. In (b) oddyears are depicted in blue, and even-years are red. . . . . . . . . 87

Figure 4.40. The Hill-Ricker growth function used for this simulation. . . . . . . 89 
Figure 4.41. A sample of the density $u_{t}(x)$ vs. $x$ for several different generations when $r=2.3$. Note immediate successors are shown across the columns, and large time differences across rows. Also note the $x$ scale varies across rows. . . . . . . . . . . . 91

Figure 4.42. A plot of the total population and domain size vs. time. Total population is blue, and domain size is violet. . . . . . . . . . . 92

Figure 4.43. Sub-figure (a) is plot of total population together with the regression line from Eq. 4.4. Sub-figure (b) is total accumulated residual square error, together with the fitted quadratic, $14.35 t+0.0139 t^{2} \ldots 94$

Figure 4.44. Sub-figure (a) shows a histogram of all density values on all meshpoints (in populated domain) from $t=900$ to $t=1100$. Sub-figure (b) shows a histogram of the 1-year total population change on 5000 randomly selected intervals of length $\ell=1.85$, also selected from years $t=900$ to $t=1100 \ldots \ldots \ldots \ldots$. . . . . . . . 97

Figure 4.45. Sub-figure (a) is plot of the sample covariance of $u_{t}\left(x_{i}\right)$ and $u_{t}\left(x_{i}+\right.$ $s$ ) for 10,000 points. $t$ is picked randomly from $900, \ldots, 1100$, and $x_{i}$ is picked randomly from the populated domain of $u_{t} . s$ is the value plotted on the x-axis. Sub-figure (b) is $\log _{10}(|S . C|$.$) vs. length.$ Values falling below the red dashed line can be considered to have no statistically significant covariance. . . . . . . . . . . 98

Figure 4.46. Sub-figure (a) shows the domain length data (red), and fitted line (dashed black). Sub-figure (b) shows the residual errors vs. time, the residual error is the difference between the data and fitted line. . 100

Figure 4.47. Yearly changes in domain length plotted vs. time. Sub-figure (b) shows a detailed view of $\Delta$ dom near the $\Delta$ dom $=0$ line. The black dashed line is the average value of $\Delta$ dom. . . . . . . . . . 101

Figure 4.48. An illustration of the calving process . . . . . . . . . . 103 
Figure 4.49. A detailed view of $u_{991}(x) \ldots \ldots$. . . . . . . . . . . . 104

Figure 4.50. An illustration of a run of gradual increases. . . . . . . . . . . . 105 


\section{CHAPTER 1 \\ INTRODUCTION}

Spatial population models are used to model the densities of a species, infectious disease, or a genetic variant of a species across both space and time. Spatial population models can include reaction-diffusion equations, and integro-difference equations.

Reaction-Diffusions equations are continuous time partial differential equation models where growth and diffusions take place simultaneously (Grinrod 1996, Zhou 2011). Typically Fickian diffusion is assumed and modeled by the Laplacian operator (Murray 2001). However some variants may assume fractional-order diffusion (El-Sayed 2009), or density dependant diffusion (Bertsch 1986). An early example of the application of Reaction-Diffusion models to population biology were by R.A. Fisher in 1937 as well as Kolmogorav et al. 1937. Fisher, for instance, used the equation

$$
\frac{\partial u}{\partial t}=d \frac{\partial^{2} u}{\partial x^{2}}+r u(1-u)
$$

to model the spread of an advantageous gene into the environment, $u$ being the fraction of the population with the advantaged gene. Fisher was able to demonstrate that the advantaged population front would move at the constant speed $2 \sqrt{d r}$, where sufficiently far in front of the invasion front $u(x)=0$ and sufficiently far behind $u(x)=1$. Slightly later Skellam (1951) used reaction-diffusion equations to model muskrat populations.

More modern work has for instance taken into account periodic spatial variation (Huang 2009). Often analysis of reaction diffusion models falls under either determine the asymptotic spreading speed on an infinite domain (Otto 2017), or determining the minimum domain size required when hostile (Dirichlet) boundary conditions are as- 
sumed (Shi 2006). If an advective term is added, reaction-diffusion equations can be used to model fish and insect populations in streams (Seo 2011, Lutsher 2011, Vasilyeva 2016, Li /Fagan/Wang 2014). This can be used ,for instance, to determine at what flow rates a species will be washed out of a river. If the density dependant per-capita growth rate is allowed to vary spatially and temporally then reaction-diffusion equations can be used to model global climate change. Much recent work has been devoted to determining conditions in which a species can and cannot persist in the face of global-warming (Bereestycki 2009, Li/Bewick 2014, Potopov 2004). Reaction-diffusion equations can be used to model the interaction between multiple species. For instance Okubo in 1989 modeled the competitive invasion of the grey squirrel against the native squirrel in Britain. Owen (2001) used reaction-diffusion models to study when and if a predator can stop an invasive prey species.

Prior to discussing Integro-difference models, it is worthwhile to have some discussion about non-spatial discrete time recursion models. Models of the form

$$
N_{t+1}=g\left(N_{t}\right)
$$

can used to model population with non-overlapping generations. Here $N$ is the population or density, $t$ is the year or season, and $g$ is a fecundity function Some examples of species well matched to these hypothesis are fruit flies (Murray 2001), and annual plant species (Edelstein-Keshet 2004).

Broadly speaking $g$ can be said to exhibit mono-stability, Allee effect (positive density dependance), or overcompensation (negative density dependance) (Schreiber 2003). A Mono-stable growth function is increasing, bounded by its tangent line at $0,(g(x) \leq$ $\left.g^{\prime}(0) x\right)$ and possesses a single stable positive equilibrium $N_{e}$ where $g\left(N_{e}\right)=N_{e}$. Any solution introduced above or below $N_{e}$ will smoothly increase or decrease respectively towards $N_{e}$. The Beverton-Holt model (Beverton 1957) is a commonly used mono-stable growth function. In the most basic form it can be derived by integrating the logistic 
growth O.D.E,$\dot{N}=r N\left(1-\frac{N}{K}\right)$ from the start to end of the season $(t=0 \ldots T)$. One obtains

$$
N_{t+1}=\frac{K e^{r T} N_{t}}{K+N_{t}\left(e^{r T}-1\right)},
$$

where $K$ is the equilibrium population.

Allee effect, or a positive dependance of the per-capita growth rate on density, was first noticed in the 1930's by the biologist Warder Allee, in an experiment involving goldfish populations (Allee 1937). There are many biological mechanisms that can lead to Allee effects:

- cooperative predation or defense (Stephens 1999)

- mate finding failures at low density (Boukal 2002, Calabrese 2004, Hopf 1985, Lindstrom 1998, Viet 1996)

- pollination failure at low densities (Groom 1998)

- predator saturation (dr Roos 1998, Wilson 1997)

There is an important distinction to be made between what is termed the weak Allee effect and the strong Allee effect (Wang 2002). Weak Allee effect is said to occur when $g^{\prime}(0)>1$ but $g^{\prime \prime}(0)>0$. In other words arbitrarily small populations continue to grow at an increasing rate until saturation is reached. With strong Allee effect $g^{\prime}(0)<$ 1 and there is some threshold value $u_{\text {allee }}$ whereby $g\left(u_{\text {allee }}\right)=u_{\text {allee }}$ and $g^{\prime}\left(u_{\text {allee }}\right)>1$. Populations introduced below this threshold perish, whereas those introduced above grow until saturation is reached. Throughout this paper we will focus on the strong Allee effect.

As an example in Boukal (2002) they discuss a 2-sex model where the rate of female pregnancy is assumed to be proportional to $M \times F$, the females are assumed to be only able to reproduce once per season, and the sex-ratio is fixed. Under these assumptions one finds the per-capita growth rate to be

$$
r\left(1-e^{-a N}\right)
$$


where $r$ is the offspring per pregnant female, and $a$ involves mate finding efficiency, length of reproductive season, and sex ratio. In the Mathusian version of this model the Allee threshold is the solution to $r\left(1-e^{-a N}\right)=1$ or $N_{\text {allee }}=\frac{\ln \left(\frac{r}{r-1}\right)}{a}$. A more realistic full model can be constructed by assuming a impulsive reproductive period at the beginning of the season followed by logistic decay, $\dot{N}=-d N-\beta N^{2}$ until the end of the season.

Overcompensation is said to occur when negative density dependant factors cause $g$ to reach some maximum value and then decrease (Schreiber 2003). One of the earliest models with overcompensation was the Ricker model (1954), which was derived from a model were adults cannibalized juveniles in fishing stocks. In units of the carrying capacity, the Ricker model is given by

$$
N_{t+1}=N_{t} \exp \left(r\left(1-N_{t}\right)\right)
$$

where $r$ is the intrinsic growth rate. The single non-trivial equilibrium is $N=1$. For $0<r<2 N=1$ is stable, for $2<r<2.69$ period doubling occurs, and for $r>2.69$ chaotic dynamics ensue. Escola et al. (2007) was able to mechanistically derive a number of other models with overcompensation by considering in season O.D.E. adult and juvenile system, where various permutations of either adults or juveniles or both preying on either adults or juveniles or bothare considered.

Some other mechanisms for overcompensation can include overcrowding in plants, for instance Deng (2012) showed that for crop plants, the yield per area of seed biomass decreased in proportion to $N^{\frac{-1}{3}}$ for super-optimal planting densities. Some other mechanisms for overcompensation include resource depletion (Tillman 1982) and environmental modification (Jones 1997).

Finally, when strong Allee effect, and overcompensation are combined it possible to have a phenomena called essential extinction occur (Schrieber 2003). Essential extinction occurs when very strong overcompenation causes large densities to be mapped 
below the Allee threshold. Formally if $u_{\max }=\max _{u>0} g(u)$ and $g\left(u_{\max }\right)<u_{\text {allee }}$ then essential extinction is said to occur. Schrieber (2001) has shown that under some rather general hypothesis involving the Schwarzian derivative, if essential extinction occurs then almost every solution initiated between $0<u_{0}<u_{\max }$ will eventually be mapped below the Allee threshold and face extinction. Here "almost every" is meant in the sense that only a set of Lebesgue measure zero does not face extinction. In other words randomly picking an initial condition, there is a $100 \%$ probability that it will face extinction.

Integro-difference equations (IDE's) can be considered as the natural spatial extension of discrete time recursion models. It is assumed that there are discrete generations and that the reproductive process takes place relatively synchronously followed by a dispersal process (Kot 1986). In the simplest case, the reproduction is assumed to be governed by a growth function that only depends on the local density, $g(u(x))$. The dispersal is governed by a probability distribution $k$, that determines the probability of an offspring (or seed) starting at point $y$ and landing at point $x$. Again assuming the simplest case, of a spatially and temporally homogenous environment and density independent dispersal, we obtain

$$
u_{t+1}(x)=\int_{-\infty}^{\infty} k(x-y) g\left(u_{t}(y)\right) \mathrm{d} y
$$

The unspecified dispersion kernel $k$ allows a very wide variety of dispersal behaviors to be modelled as compared to what is possible within the confines of reactiondiffusion framework (Zhou 2011). As is discussed in Kot (1996) many species dispersal data indicates a more Leptokurtic dispersal pattern then what would result from Gaussian dispersal implied by Fickian diffusion. Roughly speaking leptokurtic means the tails and center contain more mass then what would be expected from a Normal distribution of the same variance.

As cursory look at some of the important biological results obtained within the IDE framework:

- (Kot 1986) obtained results concerning a minimum domain size with an IDE on a 
bounded domain.

- (Van Kirk 1997) used IDE's to study persistence in fragmented habitats.

- (Fagan 2005) used an IDE model to study if the invasion of plant could be stopped by a herbivore.

- (Lutscher 2008) studied density dependant dispersal kernels within the IDE framework.

- (Zhou 2011) used an IDE model to study persistence in the face of climate change.

- (Meyer 2013) used an IDE model to study the spread of plants with seed banks.

Now we will discuss some of the more important mathematical results involving IDE's.

Weinberger $(1978,1982)$ proved some very general results for an operator $Q$ that can be considered a generalization of the left hand side of Eq. 1.1 when $g$ is a monostable growth function. Weinberger showed that there exists a minimum spreading speed , $c^{*}$, of a class of travelling waves which may generally depend on direction. Solutions introduced with compact support will travel outwards at this speed, far ahead of this invasion front, zero population will be seen, while far behind the positive equilibrium population density will be seen.

Weinberger gave a method for calculating the spreading speed based on the moment generator of the linearization of $Q$ about the 0 population (Otto 2017). For example linearizing Eq. 1.1 about $u_{t}(x)=0$ we obtain

$$
\widetilde{Q}[f(s)](x)=g^{\prime}(0) \int_{-\infty}^{\infty} k(x-s) f(s) \mathrm{d} s
$$

We then define the moment generator as

$$
\Lambda(\mu):=\widetilde{Q}\left[e^{-\mu s}\right](0)=g^{\prime}(0) \int_{-\infty}^{\infty} k(-s) e^{-\mu s} \mathrm{~d} s
$$


The forward spreading speed can then be calculated as

$$
c^{*}=\inf _{\mu>0} \frac{\ln (\Lambda(\mu))}{\mu},
$$

thus reducing the problem of determining the spreading speed to a simple minimization problem in the case that the moment generator of $k$ has a closed form.

In 1985 Roger Lui proved under rather general circumstances that monotone growth functions with Allee effects have stable travelling wave solutions of a constant speed in the IDE setting.

In 2002 Wang, Kot and Nuebert determined a easily calculable condition for when monotone growth functions with strong Allee effect either retreat $(c<0)$ or advance $(c>0)$. If $u_{\text {stab. }}$ is the stable super-Allee threshold equilibrium, then the sign of

$$
\int_{0}^{u_{\text {stab. }}} g(u)-u \mathrm{~d} u
$$

determines the sign of $c$.

In $2009 \mathrm{Li}$, Lewis and Weinberger proved that non-monotone (over-compensatory) growth functions without Allee effects have an invasion front with a constant speed. Unlike the other cases the dynamics behind the invasion front can be be quite chaotic depending on the severity of the over-compensation.

Very little work has been done on IDE's in the case that $g$ exhibits both strong Allee effect and over-compensation. Some recent numerical work by Sullivan (2017) has shown that when these effects are combined the year to year spreading speed oscillates chaotically. While previous work on unbounded domains has typically focused on spreading behaviour, in this work we will demonstrate that a very different behaviour is possible when Allee and over-compensation effects are combined. Namely, we show that under certain conditions solutions exist where the population persists but only remains in a compact location.

In Chapter 2 we introduce our general IDE model, and provide a rigorous definition of non-spreading solutions. 
In Chapter 3 we use a specific 3 parameter piecewise constant growth function and an ansatz solution to derive an algebraic conditions for the existence of equilibrium nonspreading solutions. There is some history of using simplified growth functions to obtain tractable results, for instance Kot (1996) uses a piecewise 1-step constant function with strong Allee effect to derive a closed form spreading speed, and Wang (2002) extends these results by considering a linear ramp and deriving a asymptotic approximation of the spreading speed.

For the case when $k$ is the Uniform-distribution we are able to derive an closed form algebraic condition for the existence of nonspreading equilibrium solutions. We then subsequently show that these are robust in that they exist on a full 3-dimensional volume of the parameter space. Numerical algebraic techniques are employed to show solutions also exist in full-measure for the Gaussian dispersal kernel. Since the exponential power distribution is often used to model dispersal with variable kurtosis (Fitt 1987, Wilson 1993), we also study the existence of non-spreading solutions for various exponents.

In Chapter 4.0.4-4.0.6 we use numerical methods to test the stability of the equilibrium solutions derived analytically. In the case of the Uniform dispersal kernel we produce bifurcation diagrams showing for what initial conditions solutions tend towards non-spreading solutions versus spreading solutions.

In Chapter 4.0.7 we demonstrate numerically the existence of non-spreading solutions for more realistic growth functions then the piecewise one we considered.

Pattern formation is a common topic of study in spatial population dynamics. Often pattern formations requires the interaction of multiple species. For example, Nuebert (1995) and de Roos (1998) both consider pattern formation in IDE models with predatorprey interactions. In Chapter 4.0.8 we demonstrate patch emergence in the single species model with Allee and over-compensation effects from perturbed constant initial data.

As was mentioned earlier, in non-spatial models growth functions with essen- 
tial extinction go extinct almost-surely. In Chapter 4.0.9 we demonstrate when essential extinction is coupled to spatial spread it is possible to have a persistent and expanding population. 


\section{CHAPTER 2}

\section{MODEL AND DEFINITIONS}

We consider a single species discrete time model in a one-dimensional habitat , that conforms to the biological assumptions of an integro-difference model (Kot 1986). We assume dispersion and fecundity are not spatially or temporally dependant and we assume dispersion is not density dependant. The operator $Q[\cdot]$ mapping the population density profile $u_{n}(x)$ of season $n$ to that of season $n+1$ is thus defined by

$$
u_{n+1}(x)=Q\left[u_{n}\right](x):=\left(k *\left(g \circ u_{n}\right)\right)(x)=\int_{-\infty}^{\infty} k(x-y) g\left(u_{n}(y)\right) \mathrm{d} y .
$$

Where $k(\cdot)$ is a probability density function describing dispersal, and $g(\cdot)$ is the fecundity function.

For the purposes of this paper we will limit our attention to symmetric dispersal kernels, and we will assume there is a unique inverse cumulative distribution function. We will assume the growth function exhibits both the strong Allee effect and overcompensation. Mathematically we will define strong Allee effect to mean there exists a $u_{a}>0$ such that for all $u \in\left(0, u_{a}\right), g(u)<u$. We will define over-compensation to mean there exists some $u_{o c}>0$ such that $g(u)$ is non-decreasing on $\left(0, u_{o c}\right)$, and $g(u)$ is nonincreasing on $\left(u_{o c}, \infty\right)$, additionally we will stipulate $g(u)<g\left(u_{o c}\right)$ for all $u>u_{o c}$. By boundedness of $g(u)$ and properties of convolution, we see $Q\left[u_{n}\right](x)$ will be bounded and continuous.

We provide the following rigourous definition of non-spreading solutions: 
DEFINITION 2.1. Non-spreading Solutions

We say a solution of Model 2.1, $\left\{u_{n}\right\}_{n=0}^{\infty}$, is non-spreading if:

(i) There exists a $\delta>0$ such that for all $n \geq 0$ there exists a $x_{n}$ such that $u_{n}\left(x_{n}\right)>\delta$.

(ii) For every $\varepsilon>0$ there exists $a \sigma>0$ such that $u_{n}(x)<\varepsilon$ for all $n \geq 0$ and $|x|>\sigma$.

Condition (i) can be interpreted as a persistence condition, namely that for every generation there will be at least some point where the density exceeds the threshold $\delta$. Condition (ii) can be interpreted as a non-spreading or localization condition, where outside of a fixed interval the density will remain arbitrarily small. 


\section{CHAPTER 3 \\ EQUILIBRIUM SOLUTIONS-ANALYTICAL RESULTS}

To demonstrate the existence of non-spreading and persistent solutions when strong Allee effect and over-compensation are present, we first analytically demonstrate the existence of spatially localized equilibrium solutions for a particular class of parameterized fecundity functions. If $u_{e}$ is a fixed point of $Q$ and is localized in the sense of (ii) in definition 2.1 then clearly it will satisfy the persistence condition.

In this section we will first give some preliminary definitions and then present a theorem for the existence of equilibriums within our particular class of fecundity functions. The theorem gives algebraic conditions in terms of the cumulative distribution function of $k(\cdot)$ as well as the parameters of the growth function. When these conditions are satisfied it guarantees the existence of equilibria.

We define our growth function to be a 3-level piecewise constant function. By way of scaling, we may in generality assume that the Allee threshold occurs at $u=1$. The growth function, pictured in Figure 3.1, is then given by

$$
g(u):=\left\{\begin{array}{ll}
0, & 0 \leq u \leq 1 \\
n_{2}, & 1<u<n_{1} \\
n_{0}, & u \geq n_{1}
\end{array} .\right.
$$

The growth function is thus determined by the 3 parameters, $n_{0}, n_{1}, n_{2}$. To exhibit over-compensation and have the possibility of non-decaying solutions, the parameters must satisfy the inequalities $n_{0}<n_{1}<n_{2}$ and $1<n_{1}, n_{2}$. 


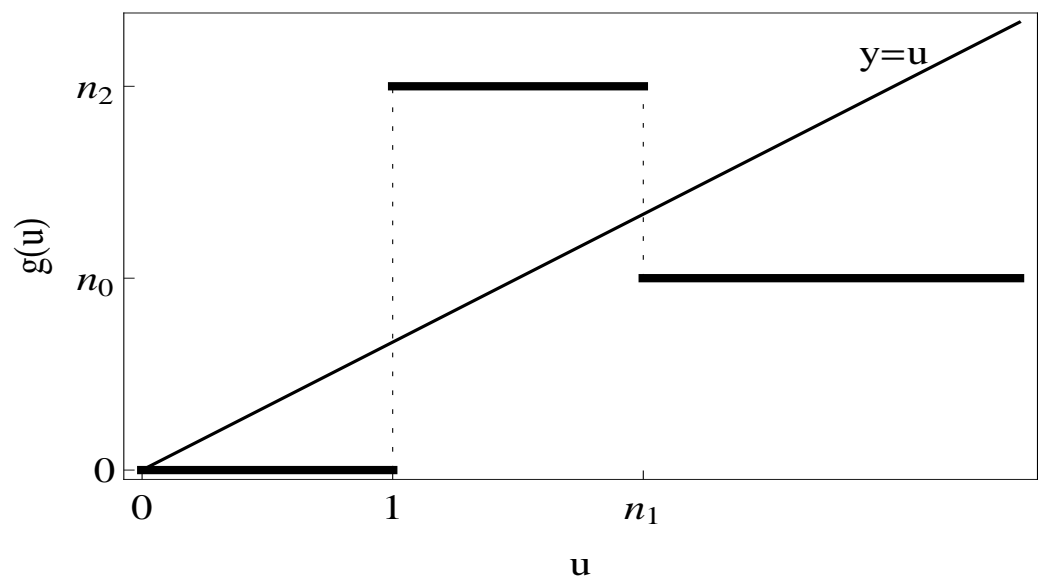

Figure 3.1: fecundity function and the line $y=u$

To determine conditions for the existence of equilibriums, we invoke the following ansatz about the form of the composition of the growth function with the equilibrium. We assume $g\left(u_{e}(x)\right)$ takes the form pictured in Figure 3.2, and is given by the equation

$$
g\left(u_{e}(x)\right)=G_{\{a, b\}}(x):= \begin{cases}n_{0}, & |x|<a \\ n_{2}, & a<|x|<b \\ 0, & |x|>b\end{cases}
$$

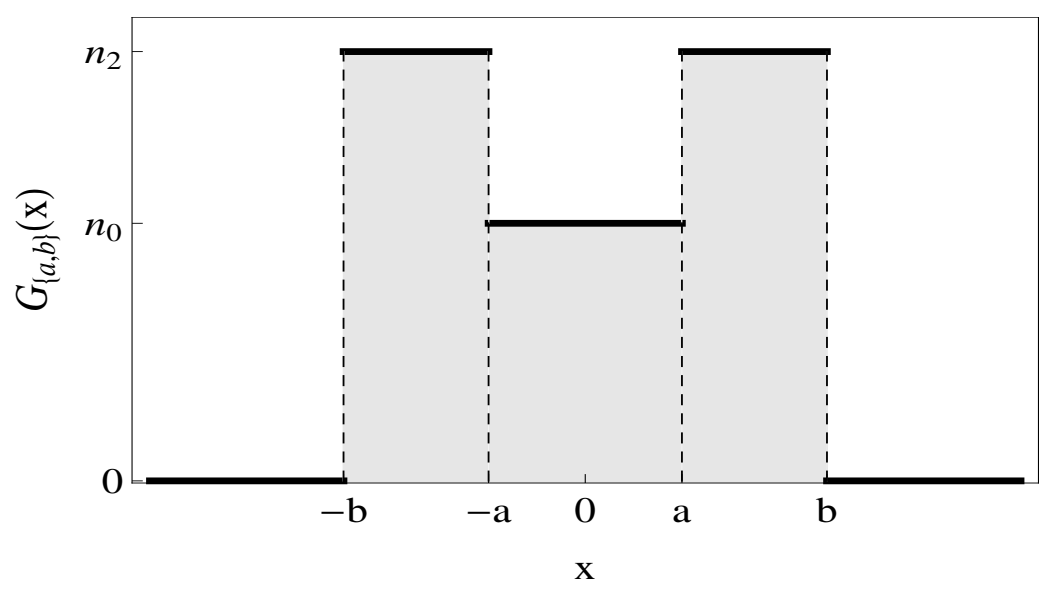

Figure 3.2: plot of $G_{\{a, b\}}(x)$ 
We see this form of $g\left(u_{e}(x)\right)$ is consistent with $u_{e}$ being a symmetric, unimodal function where the following conditions hold:

\section{Condition (C1)}

1. $u_{e}( \pm a)=n_{1}$

2. $u_{e}( \pm b)=1$

3. $u_{e}(0)>n_{1}$.

Noting if $u_{e}$ is truly a fixed point of $Q$ and $g\left(u_{e}(x)\right)=G_{\{a, b\}}$ then $u_{e}(x)=(k *$ $\left.G_{\{a, b\}}\right)(x)$ and therefore Condition C1 implies

1. $\left(k * G_{\{a, b\}}\right)(a)=n_{1}$

2. $\left(k * G_{\{a, b\}}\right)(b)=1$

3. $\left(k * G_{\{a, b\}}\right)(0)>n_{1}$.

We define $K(x)$ to be the cumulative distribution function of $k(x)$, i.e. $K(x)=$ $\int_{-\infty}^{x} k(s) \mathrm{d} s$. With the addition of the following definitions we will have completed the preliminaries for our sufficiency theorem regarding the existence of equilibriums.

$$
\begin{aligned}
A(a, b) & :=\left(k * G_{\{a, b\}}\right)(a)-n_{1} \\
& =-n_{1}-\frac{\left(n_{2}+n_{0}\right)}{2}+n_{2}(K(a+b)+K(b-a))-\left(n_{2}-n_{0}\right) K(2 a), \\
B(a, b) & :=\left(k * G_{\{a, b\}}\right)(b)-1 \\
& =-1+n_{2}\left(K(2 b)-\frac{1}{2}\right)+\left(n_{2}-n_{0}\right)(K(b-a)-K(b+a)), \\
C(a, b) & :=\left(k * G_{\{a, b\}}\right)(0)-n_{1} \\
& =-\left(n_{1}+n_{0}\right)+2 n_{2} K(b)-2\left(n_{2}-n_{0}\right) K(a) .
\end{aligned}
$$


THEOREM 3.1. If there exists an $a, b$ where $0<a<b$ such that

$$
A(a, b)=0, \quad B(a, b)=0, \quad C(a, b)>0
$$

and furthermore the function

$$
u_{e}(x):=\int k(x-y) G_{\{a, b\}}(y) \mathrm{d} y
$$

is such that $u_{e}(x)>n_{1}$ if $|x|<a, 1<u_{e}(x)<n_{1}$ if $a<|x|<b$, and $u_{e}(x)<1$ when $|x|>b$ then $u_{e}(x)$ is an equilibrium of $Q[\cdot]$.

Proof: Consider there exists an $a, b$ such that $0<a<b$ and

$$
A(a, b)=0, B(a, b)=0, C(a, b)>0 .
$$

Letting $u_{1}(x)=\left(k * G_{\{a, b\}}\right)(x)$, it then follows from the definition of $A, B, C$ in Eq. 3.3 that $u_{1}(0)>n_{1}, u_{1}(a)=n_{1}, u_{1}(b)=1$. Therefore if

- for all $x \in(-a, a), u_{1}(x)>n_{1}$

- for all $x \in(-b,-a) \cup(a, b), 1<u_{1}(x)<n_{1}$

- for all $|x|>b, u_{1}(x)<1$

it then follows by the definition of $g(\cdot)$ in Eq. 3.1 that $g\left(u_{1}(x)\right)=G_{\{a, b\}}(x)$. We therefore see that $Q\left[u_{1}\right]=\left(k * g \circ u_{1}\right)=\left(k * G_{\{a, b\}}\right)=u_{1}$ and therefore $u_{1}$ is an equilibrium of $Q$.

To determine further results the dispersal kernel must be specified. In the following sections we analyze the case of the uniform and Gaussian dispersal kernels. 


\subsubsection{Uniform dispersal kernel}

We consider $k(\cdot)$ to be the symmetric uniform distribution with support $\left(-\frac{1}{2}, \frac{1}{2}\right)$,

$$
k(x)= \begin{cases}0, & |x|>\frac{1}{2} \\ 1, & |x| \leq \frac{1}{2} .\end{cases}
$$

By scaling argument it can be shown the existence or non-existence of equilibria is unaffected by choice of support, therefore the support of $k,\left(-\frac{1}{2}, \frac{1}{2}\right)$, can be considered fully general. While perhaps not totally biologically realistic, this may be considered the limiting case of several families of distributions, as they are made more platykurtic while fixing the variance. For instance, the exponential power distribution $\frac{\beta \exp \left(-\frac{|x|^{\beta}}{\alpha^{\beta}}\right)}{2 \alpha \Gamma\left(\frac{1}{\beta}\right)}$ converges almost-everywhere to $U\left(-\frac{1}{2}, \frac{1}{2}\right)$ as $\beta \rightarrow \infty$ with $\alpha=\frac{1}{2}$.

We are able to offer a simple characterization for when parameters $n_{0}, n_{1}, n_{2}$ have an associated equilibrium. We first define the following functions of $n_{0}, n_{1}, n_{2}$ :

$$
\begin{aligned}
& \hat{a}\left(n_{0}, n_{1}, n_{2}\right):=\frac{2 n_{0}\left(n_{1}-n_{2}\right)+n_{2}\left(2-2 n_{1}+n_{2}\right)}{4\left(n_{2}-n_{0}\right)^{2}} \\
& \hat{b}\left(n_{0}, n_{1}, n_{2}\right):=\frac{2 n_{0}^{2}+n_{2}\left(6-2 n_{1}+n_{2}\right)+2 n_{0}\left(n_{1}-2 n_{2}-2\right)}{4\left(n_{2}-n_{0}\right)^{2}} .
\end{aligned}
$$

We also define $\mathscr{A}$ to be the open triangular region in the $(a, b)$ plane with vertices $\left(0, \frac{1}{2}\right),\left(\frac{1}{4}, \frac{3}{4}\right),\left(\frac{1}{4}, \frac{1}{4}\right) . \mathscr{A}$ is depicted in Figure 3.3. 


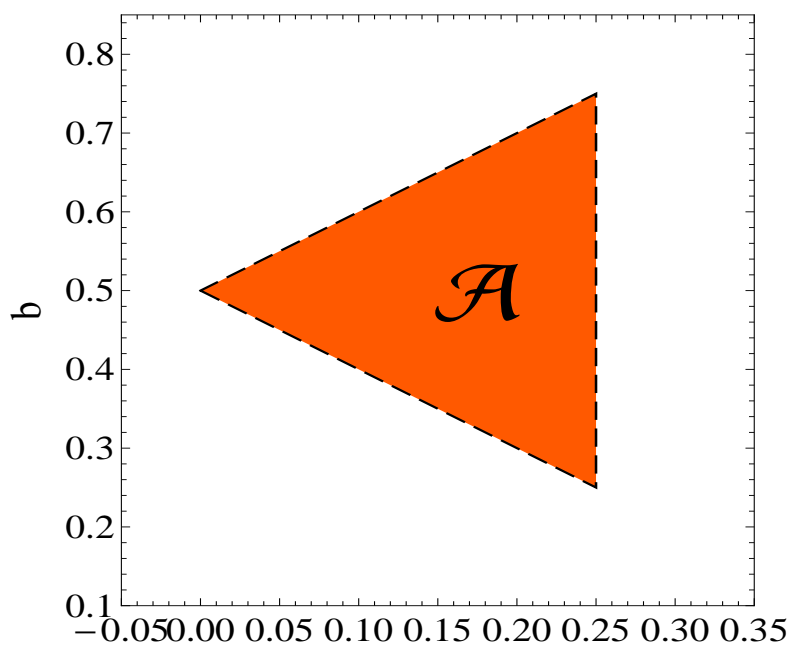

a

Figure 3.3: Region $\mathscr{A}$ of the $(a, b)$ plane.

The following theorem then characterizes the equilibria.

THEOREM 3.2. If the point $\left(\hat{a}\left(n_{0}, n_{1}, n_{2}\right), \hat{b}\left(n_{0}, n_{1}, n_{2}\right)\right) \in \mathscr{A}$ then there exists a unique equilibrium of the form given in Eq. 3.2.

Furthermore $u_{e}(x)=\left(k * G\left\{\hat{a}\left(n_{0}, n_{1}, n_{2}\right), \hat{b}\left(n_{0}, n_{1}, n_{2}\right)\right\}\right)(x)$.

Proof:

wish to derive the conditions on $n_{0}, n_{1}, n_{2}$ where the conditions in Theorem 3.1 will be satisfied for the uniform dispersal kernel. We first note that in equations $A, B, C$ (Eq. 3.3), $a$ and $b$ only appear in the arguments of $K(\cdot)$ in the form $a+b, 2 b, 2 a, b-$ $a, b, a$.

Since $a+b, 2 b, 2 a, b-a, b, a$ are all positive and $K(\cdot)$ is piecewise defined as 


$$
K(x)= \begin{cases}0, & x<\frac{-1}{2} \\ \frac{1}{2}+x, & \frac{-1}{2} \leq x \leq \frac{1}{2} \\ 1, & x>\frac{1}{2},\end{cases}
$$

we see it is necessary to make assumptions about if $a+b, 2 b, 2 a, b-a, b, a$ are less then or greater then $\frac{1}{2}$ to determine which sub-function of $K(\cdot)$ is applicable in Eq. (3.3). In Figure 3.4 the different colored regions correspond to different values for the Boolean valued vector $\left\{a+b>\frac{1}{2}, 2 b>\frac{1}{2}, 2 a>\frac{1}{2}, b-a>\frac{1}{2}, b>\frac{1}{2}, a>\frac{1}{2}\right\}$.

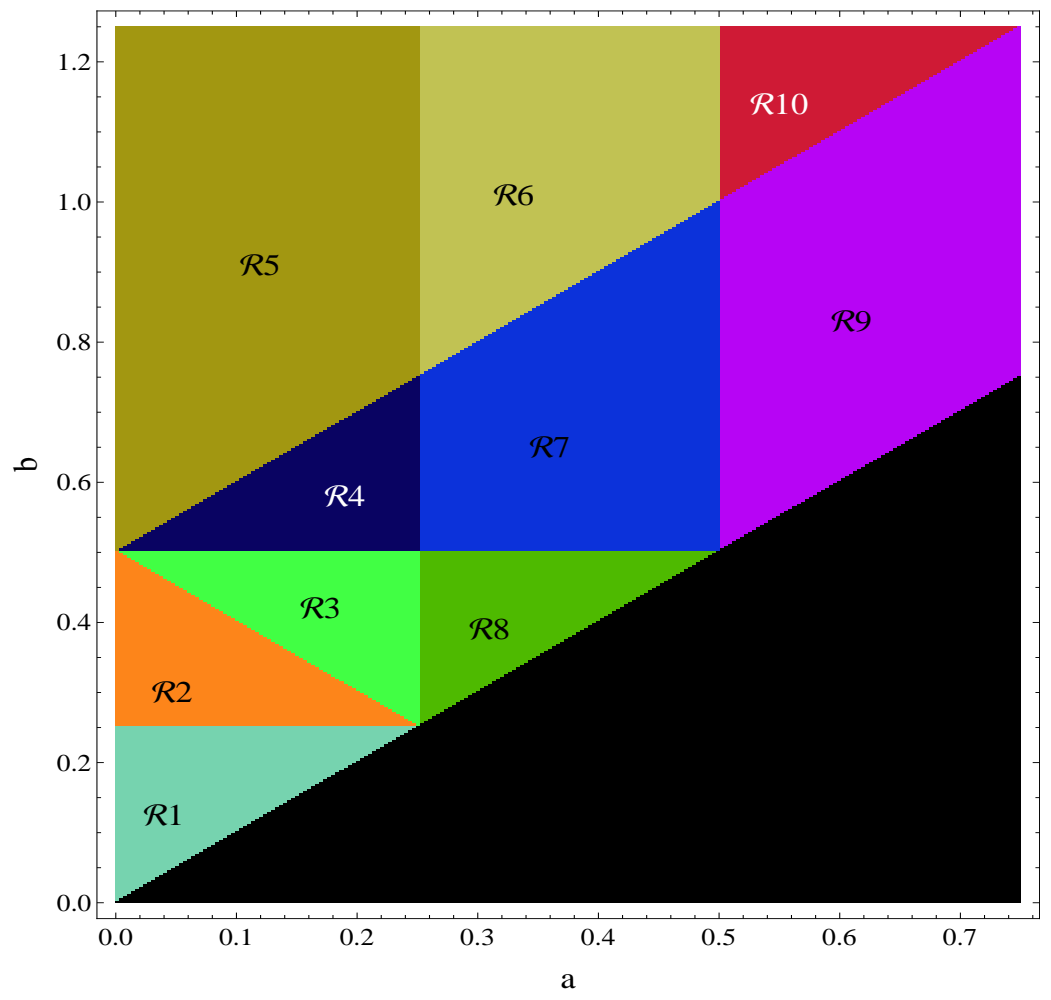

Figure 3.4: Regions of the $(a, b)$ plane where the Boolean vector $\left\{a+b>\frac{1}{2}, 2 b>\frac{1}{2}, 2 a>\right.$ $\left.\frac{1}{2}, b-a>\frac{1}{2}, b>\frac{1}{2}, a>\frac{1}{2}\right\}$ takes distinct values.

For each region we make the appropriate substitution for the sub-functions of $K(\cdot)$ and attempt to solve the system $A(a, b)=0, B(a, b)=0$. We find that for regions 
$\mathscr{R}_{1}, \mathscr{R}_{5}, \mathscr{R}_{6}, \mathscr{R}_{7}, \mathscr{R}_{8}, \mathscr{R}_{9}, \mathscr{R}_{10}$ the resulting system, which is linear in $a, b$, has a zero determinant, and thus generally no solutions.

For region $\mathscr{R}_{2}$ the resulting system $A(a, b)=0, B(a, b)=0$ becomes

$$
\begin{aligned}
& 2\left(n_{2}-n_{0}\right) a-2 n_{2} b=-n_{1} \\
& 4\left(n_{2}-n_{0}\right) a=n_{2}-2 .
\end{aligned}
$$

The solution to this system is

$$
a=\frac{n_{2}-2}{4\left(n_{2}-n_{0}\right)}, b=\frac{n_{2}+2 n_{1}-2}{4 n_{2}} .
$$

However when this result is substituted into $C(a, b)$ with the appropriate assumptions, it is found $C(a, b)=0$ thus violating the hypothesis of Theorem 3.1 and there are not any valid equilibriums.

For region $\mathscr{R}_{3}$ and $\mathscr{R}_{4}$ the resulting system for $A(a, b)=0, B(a, b)=0$ becomes

$$
\begin{aligned}
& \left(2 n_{0}-3 n_{2}\right) a+n_{2} b=-\frac{n_{2}}{2}+n_{1} \\
& \quad\left(n_{0}-n_{2}\right) a+\left(n_{2}-n_{0}\right) b=1-\frac{n_{0}}{2} .
\end{aligned}
$$

The unique solution to the system, which we use to define the functions $\hat{a}\left(n_{0}, n_{1}, n_{2}\right), \hat{b}\left(n_{0}, n_{1}, n_{2}\right)$ is given by

$$
\begin{aligned}
& a=\hat{a}\left(n_{0}, n_{1}, n_{2}\right):=\frac{2 n_{0}\left(n_{1}-n_{2}\right)+n_{2}\left(2-2 n_{1}+n_{2}\right)}{4\left(n_{2}-n_{0}\right)^{2}} \\
& b=\hat{b}\left(n_{0}, n_{1}, n_{2}\right):=\frac{2 n_{0}^{2}+n_{2}\left(6-2 n_{1}+n_{2}\right)+2 n_{0}\left(n_{1}-2 n_{2}-2\right)}{4\left(n_{2}-n_{0}\right)^{2}} .
\end{aligned}
$$

When $C(a, b)$ is evaluated at $\left(\hat{a}\left(n_{0}, n_{1}, n_{2}\right), \hat{b}\left(n_{0}, n_{1}, n_{2}\right)\right)$ and simplified with the assumption that $\left(\hat{a}\left(n_{0}, n_{1}, n_{2}\right), \hat{b}\left(n_{0}, n_{1}, n_{2}\right)\right) \in \mathscr{R}_{3} \cup \mathscr{R}_{4}$ it is found $C(\hat{a}, \hat{b})>0$. Thus the characterization for the existence of equilibriums with the uniform kernel becomes: 
There exists an equilibrium if $\left(\hat{a}\left(n_{0}, n_{1}, n_{2}\right), \hat{b}\left(n_{0}, n_{1}, n_{2}\right)\right) \in \mathscr{R}_{3} \cup \mathscr{R}_{4}$.

The equilibriums are robust in the parameter space $\left\{\left(n_{0}, n_{2}, n_{3}\right) \in \mathbb{R}^{3} \mid n_{2}>n_{1}>\right.$ $\left.n_{0} n_{1}, n_{2}>1, n_{0}>0\right\}$ in the sense that they exists on set of full-measure in the parameter space. To demonstrate this, in Figure 3.5 we show a region plot of where equilibriums exist for constant $n_{2}$ slices of the parameter space. 

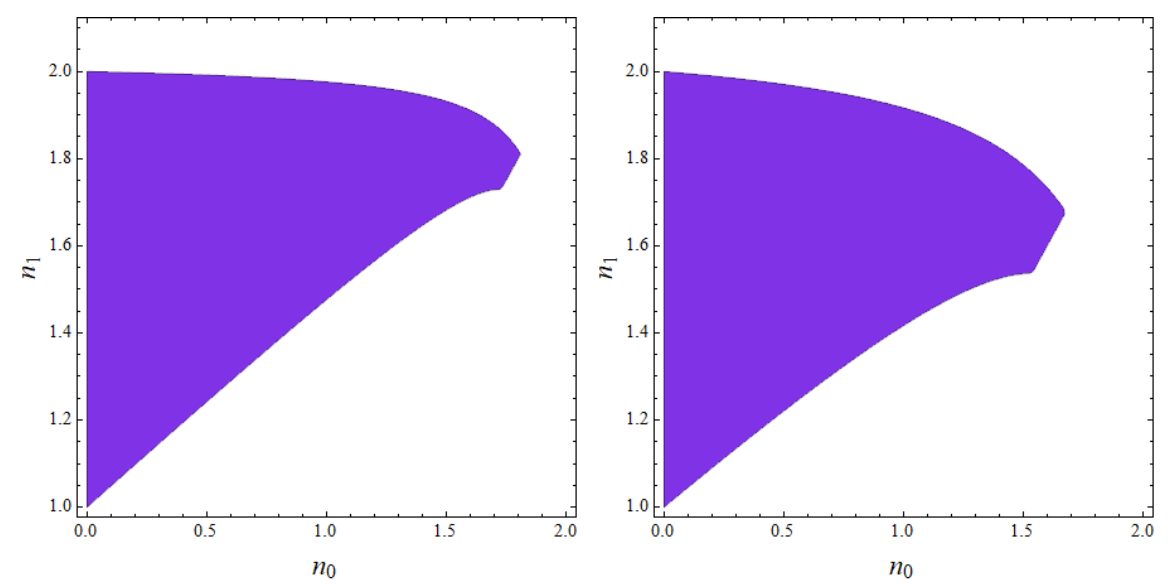

(a) $n_{2}=2.05$

(b) $n_{2}=2.25$
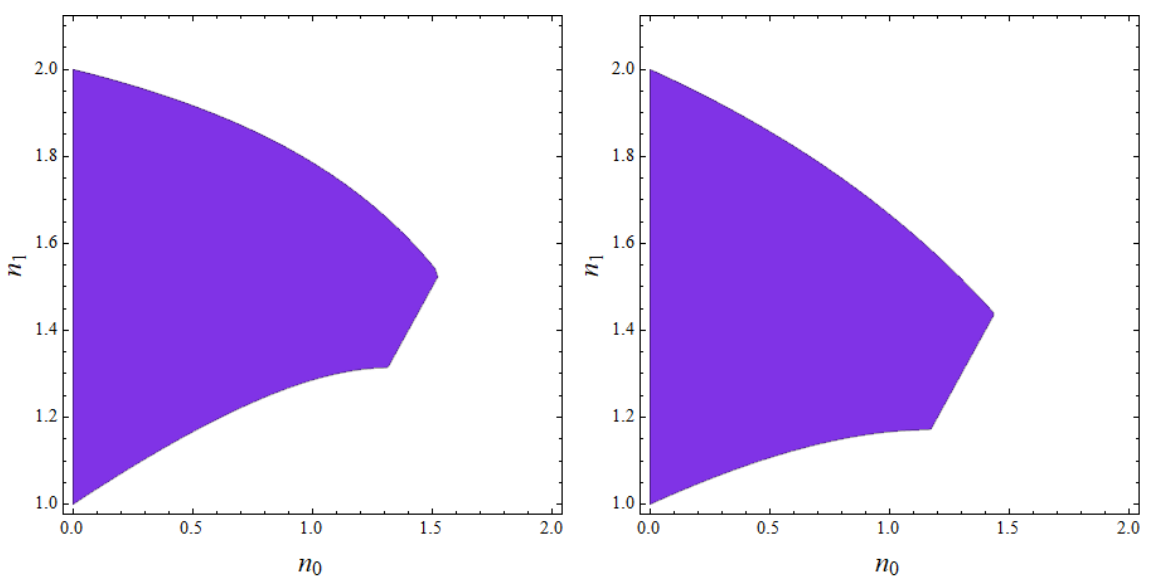

(c) $n_{2}=2.75$

(d) $n_{2}=4$

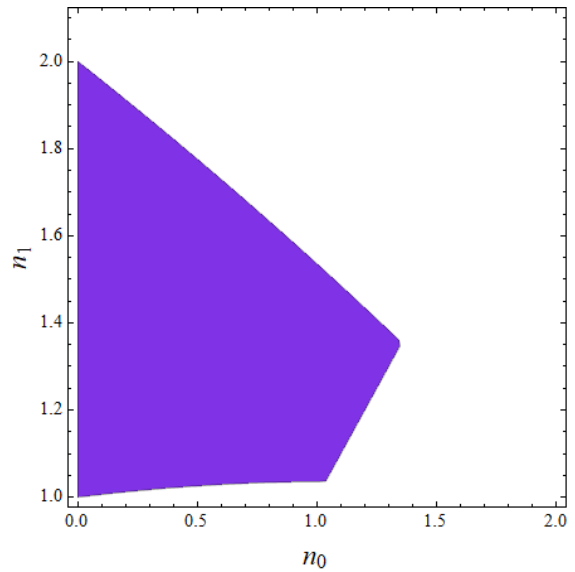

(e) $n_{2}=15$

Figure 3.5: Constant $n_{2}$ slices of parameter space. The shaded regions are parameters values where an equilibrium solution exists. 
A typical equilibrium is depicted in Figure 3.6. For the parameter values depicted $\hat{a}=0.2275$ and $\hat{b}=0.5775$. We see the function is a linear spline, as would be expected from the convolution of two piecewise constant functions. The support for $u_{e}$ is of course compact, as would be expected since both $k$ and $G_{\{\hat{a}, \hat{b}\}}$ have compact support.

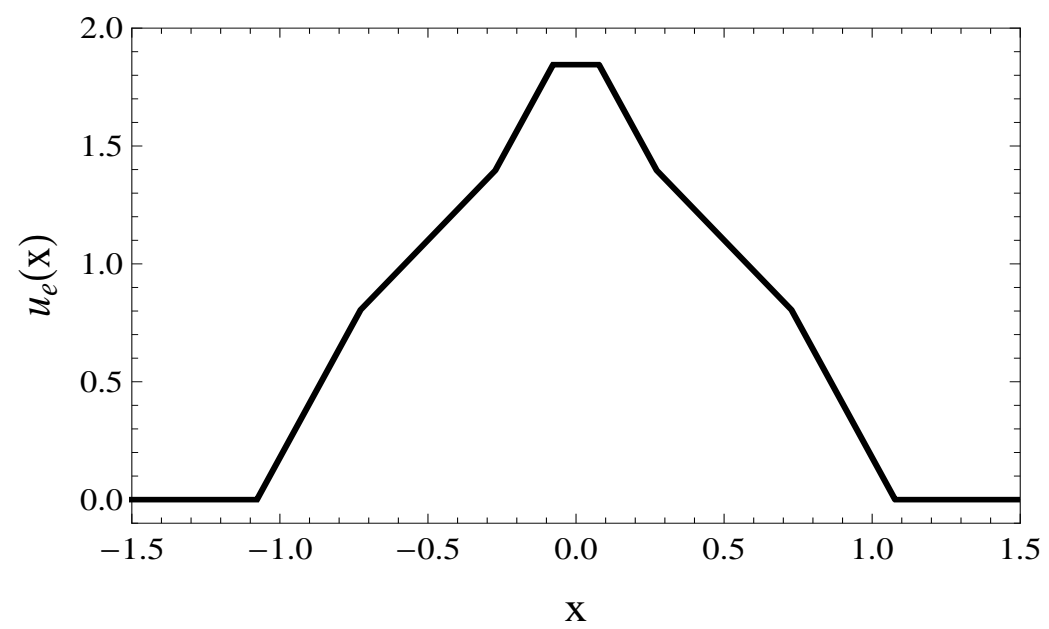

Figure 3.6: The equilibrium solution with parameter values $\left(n_{0}, n_{1}, n_{2}\right)=(1.3,1.5,2.3)$

\subsubsection{Gaussian dispersal kernel}

We consider the case where $k(\cdot)$ is the Gaussian kernel. This is consistent with the biologically realistic assumptions of a random diffusion process with a fixed stopping time. Since, as with the uniform case, scaling does not effect the existence/non-existence of equilibria for a given set of parameters, we choose to set the variance to $\frac{1}{12}$ to match the variance and proximately the length scale of the uniform case. With this choice, $k(x)=\sqrt{\frac{6}{\pi}} e^{-6 x^{2}}$.

With this definition of $k(x)$, Eq. 3.3 becomes 


$$
\begin{aligned}
& A(a, b)=-n_{1}+\frac{\left(n_{0}-n_{2}\right)}{2} \operatorname{erf}(2 \sqrt{6} a)+\frac{n_{2}}{2}(\operatorname{erf}(\sqrt{6}(a+b))+\operatorname{erf}(\sqrt{6}(b-a))) \\
& B(a, b)=-1+\frac{n_{2}}{2} \operatorname{erf}(2 \sqrt{6} b)+\frac{\left(n_{2}-n_{0}\right)}{2}(\operatorname{erf}(\sqrt{6}(b-a))-\operatorname{erf}(\sqrt{6}(b+a)))
\end{aligned}
$$

$$
C(a, b)=-n_{1}+\left(n_{0}-n_{2}\right) \operatorname{erf}(\sqrt{6} a)+n_{2} \operatorname{erf}(\sqrt{6} b)
$$

Where $\operatorname{erf}(x)=\int_{0}^{x} \frac{2}{\sqrt{\pi}} e^{-s^{2}} \mathrm{~d} s$.

With the definitions of $A, B$, and $C$ given in Eq. 3.5, it is difficult to develop analytic conditions to determine when the algebraic system given in Theorem 3.1 has solutions for a given set of parameters. We instead rely on numerical methods to make this determination. Depending on how often the curves $A(a, b)=0, B(a, b)=0$ intersect in the region of the $(a, b)$ plane where $C(a, b)>0$, we find that there are parameter values for which there are 0,1 , or 2 distinct $(a, b)$ satisfying Theorem 3.1. This is illustrated in Figure 3.7. In the case of sub-figure (c), the two distinct solutions, correspond to two distinct equilibria for that set of parameters. In Figure 3.7 we use coordinates $(b-a, b+$ a) for the purpose of allowing better visualization of the intersection(s) of the curves. 


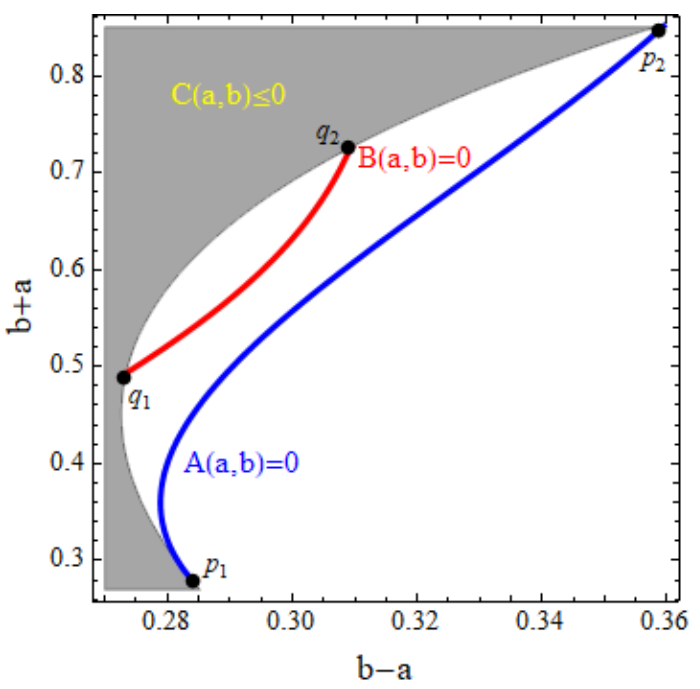

(a) $n_{0}=1.2, n_{1}=1.55, n_{2}=2.3$

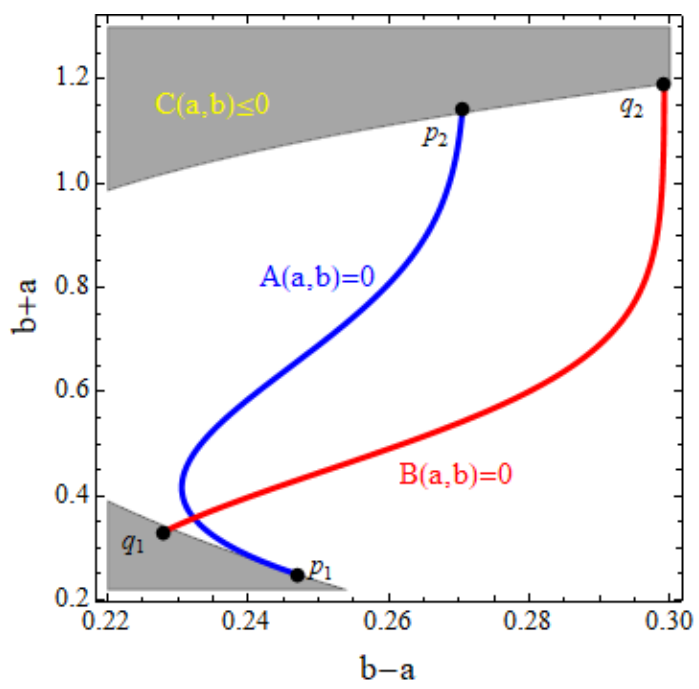

(b) $n_{0}=1.3, n_{1}=1.4, n_{2}=2.3$

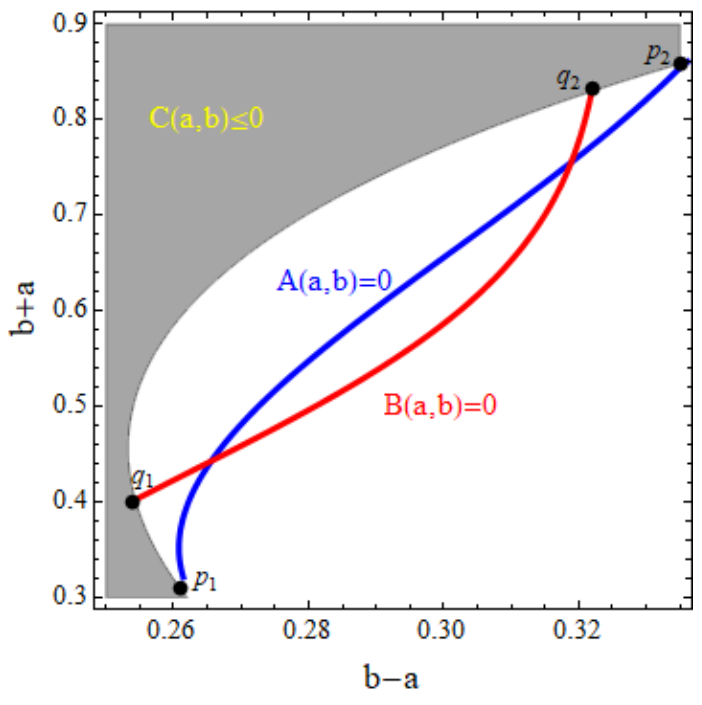

(c) $n_{0}=1.15, n_{1}=1.48, n_{2}=2.3$

Figure 3.7: Sub-Figure (a) illustrates a set of parameters having no solution to the algebraic system in Theorem 3.1, and thus no equilibria. Sub-Figure (b) illustrates a set of parameters having a single solution, and thus a unique equilibrium. In sub-figure (c) we see a set of parameters where there are two distinct equilibria.

Similar to the case of the uniform kernel, we find that parameter values with two distinct equilibria and those with a single equilibria, both occupy a set of full measure in the parameter space $\left\{\left(n_{0}, n_{1}, n_{2}\right) \in \mathbb{R}^{3} \mid n_{0}<n_{1}<n_{2}\right.$ and $\left.n_{1}, n_{2}>1\right\}$. In Figure 3.8 we 
delineate the regions with single and double equilibria. To make the region plot in Figure 3.8, we developed an algorithm that takes $\left(n_{0}, n_{1}, n_{2}\right)$ as an input and returns the number of equilibria for those parameter values. The algorithm first determines where the $A=0$ and $B=0$ curves intersect the $C=0$ curve, these are the points labeled $p_{1}, p_{2}, q_{1}, q_{2}$ in Figure 3.7. In Figure 3.7,(a) and (c) we see $q_{1}$ and $q_{2}$ are nested between $p_{1}$ and $p_{2}$ along the $C=0$ curve. By the continuity of the $A=0, B=0$ curves, with this condition it is only possible to have either zero-points where $A=0$ intersects $B=0$, as in sub-figure (a), or to have two-points where $A=0$ intersects $B=0$, as in sub-figure (c). If this condition is detected, the algorithm performs constrained numerical minimization of $A^{2}+B^{2}$ with $C>0$. If the minimum of $A^{2}+B^{2}$ is less the $10^{-6}$ the algorithm returns 2 , as it is not possible to have only one intersection in this case. If the minimum is greater then $10^{-6}$ then the algorithm returns 0 . If the algorithm determines only single $q$ lies between $p_{1}$ and $p_{2}$ on the $c=0$ curve, such as in sub-figure (b), then the only possibility is that $A=0$ and $B=0$ have only 1 intersection in the $C>0$ region, so the algorithm returns 1 . 


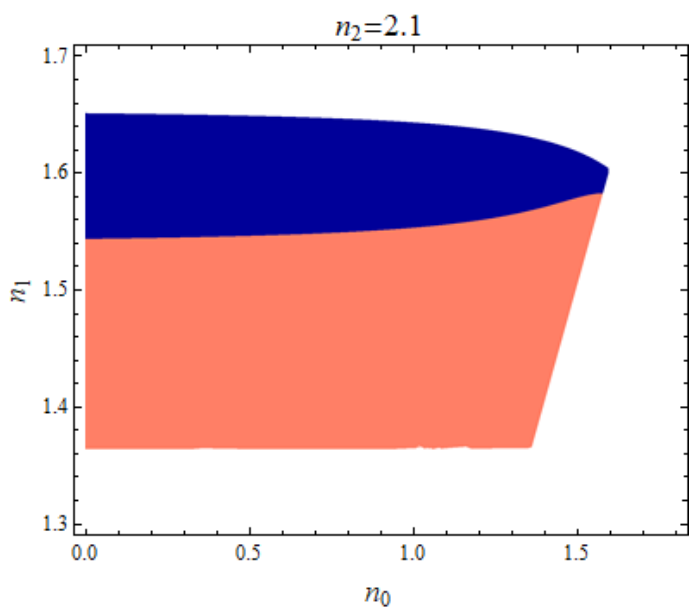

(a)

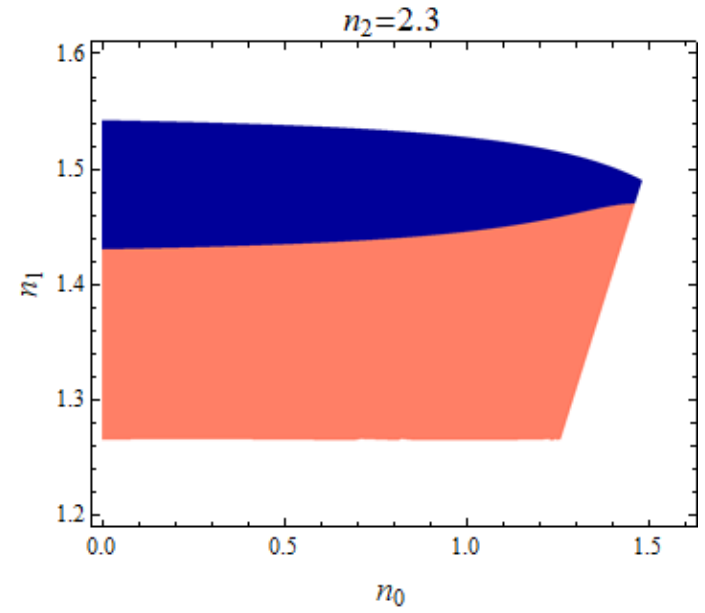

(b)

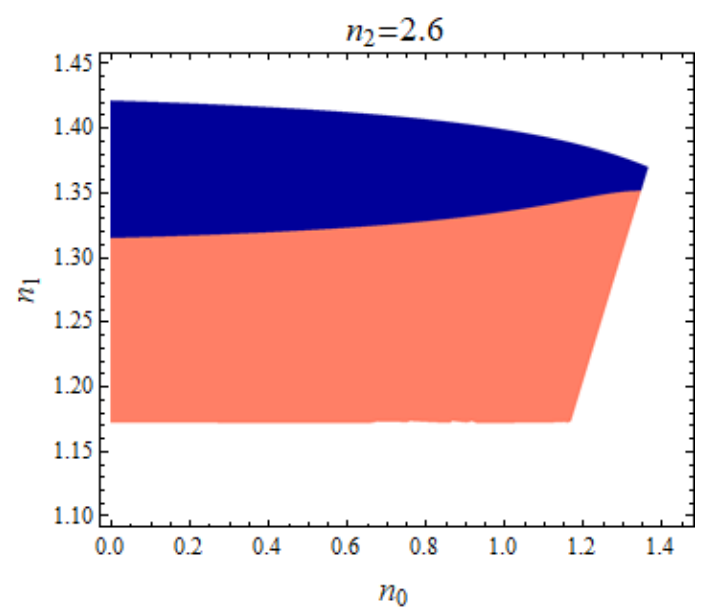

(c)

Figure 3.8: Constant $n_{2}$ slices of parameter space. The salmon colored regions are parameter values with a single equilibria. The blue regions are parameter values with 2 distinct equilibria.

In Figure 3.9 typical equilibria for the gaussian kernel are depicted. In sub-figure (a), the parameter values have a single unique equilibrium. The value of $a$ and $b$ are 0.0626 and 0.2949 respectively. In sub-figure (b), there are two distinct equilibria. For the red equilibrium curve $a$ and $b$ are 0.0887 and 0.3543 respectively. For the blue equilibrium curve $a$ and $b$ are 0.2184 and 0.5372 respectively. 


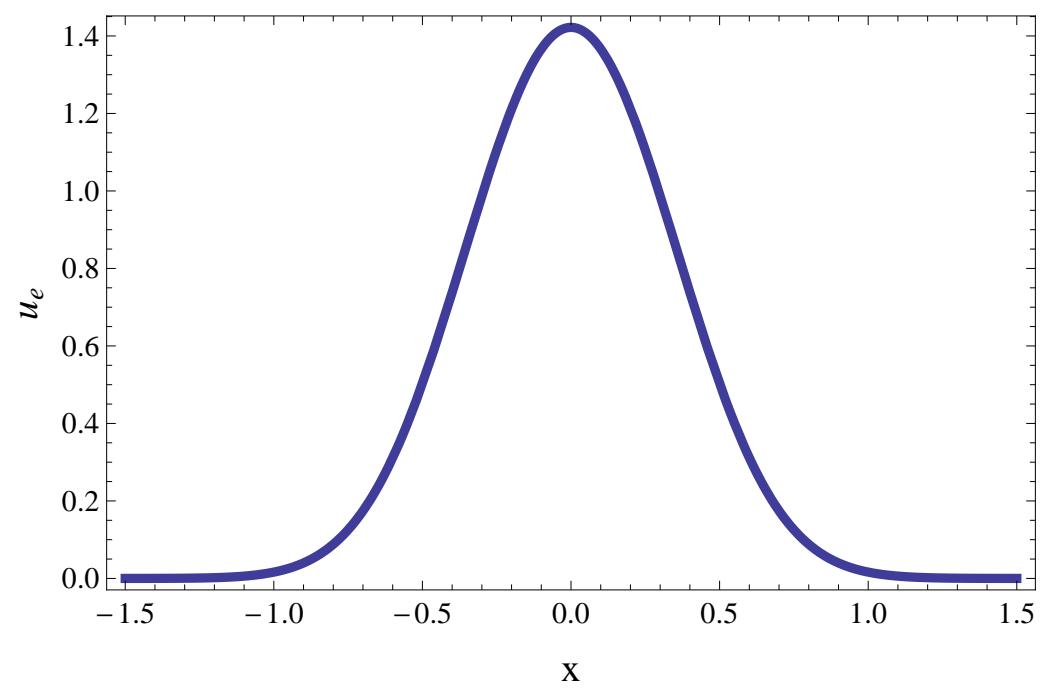

(a) $n_{0}=1.3, n_{1}=1.4, n_{2}=2.3$

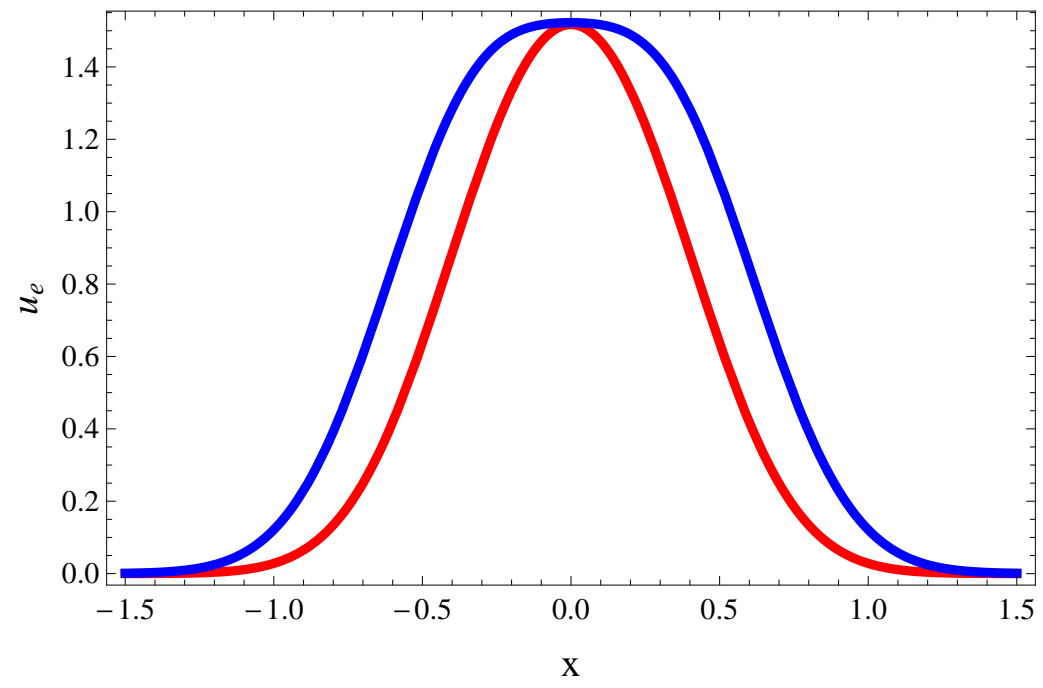

(b) $n_{0}=1.15, n_{1}=1.48, n_{2}=2.3$

Figure 3.9: Typical equilibrium solutions for the Gaussian dispersal kernel. In sub-figure (a) the parameters have a unique equilibria. In sub-figure (b) the parameters have two distinct equilibria.

\subsubsection{Exponential power distribution}

Thus far we have considered the Uniform and Gaussian kernels with variance $\frac{1}{12}$. Kurtosis, which is the ratio of the 4 th central moment to the square of the variance 
(Casella 2002), can be considered as a measure of how heavy the tails of a distribution are for a fixed variance. For instance the kurtosis of the uniform distribution is 1.8 while for the Gaussian the kurtosis is 3, reflecting what might be expected regarding the relative weights of the tails. To better understand the effect of kurtosis we consider $k(\cdot)$ to be the exponential power distribution, whose probability density function is

$$
k(x)=\frac{\gamma^{\frac{1}{\gamma}} \exp \left(\frac{-\left|\frac{x}{\sigma}\right|^{\gamma}}{\gamma}\right)}{2 \sigma \Gamma\left(1+\frac{1}{\gamma}\right)} .
$$

It is well defined for any $\gamma>0$ and $\sigma>0$ but we will only consider $\gamma \geq 1$. For the variance to be $\frac{1}{12}$,

$$
\sigma=\frac{\sqrt{\Gamma\left(\frac{1}{\gamma}\right)}}{2 \sqrt{3} \gamma^{\frac{1}{\gamma}} \sqrt{\Gamma\left(\frac{3}{\gamma}\right)}}
$$

It is worth noting that when $\gamma=1, \gamma=2$, and $\gamma \rightarrow \infty$ the exponential power distribution is the Laplace, Gaussian, and Uniform distribution, respectively. In Figure 3.10-a, we plot the kurtosis as a function of $\gamma$ where we see kurtosis is a decreasing function of $\gamma$ and the value asymptotically approaches that of the uniform kernel. The term leptokurtic is applied to the heavier tailed distributions where $\gamma<2$, and platykurtic is applied to those with lighter tails where $\gamma>2$. In Figure 3.10-b we plot the probability density functions for several values of $\gamma$. 


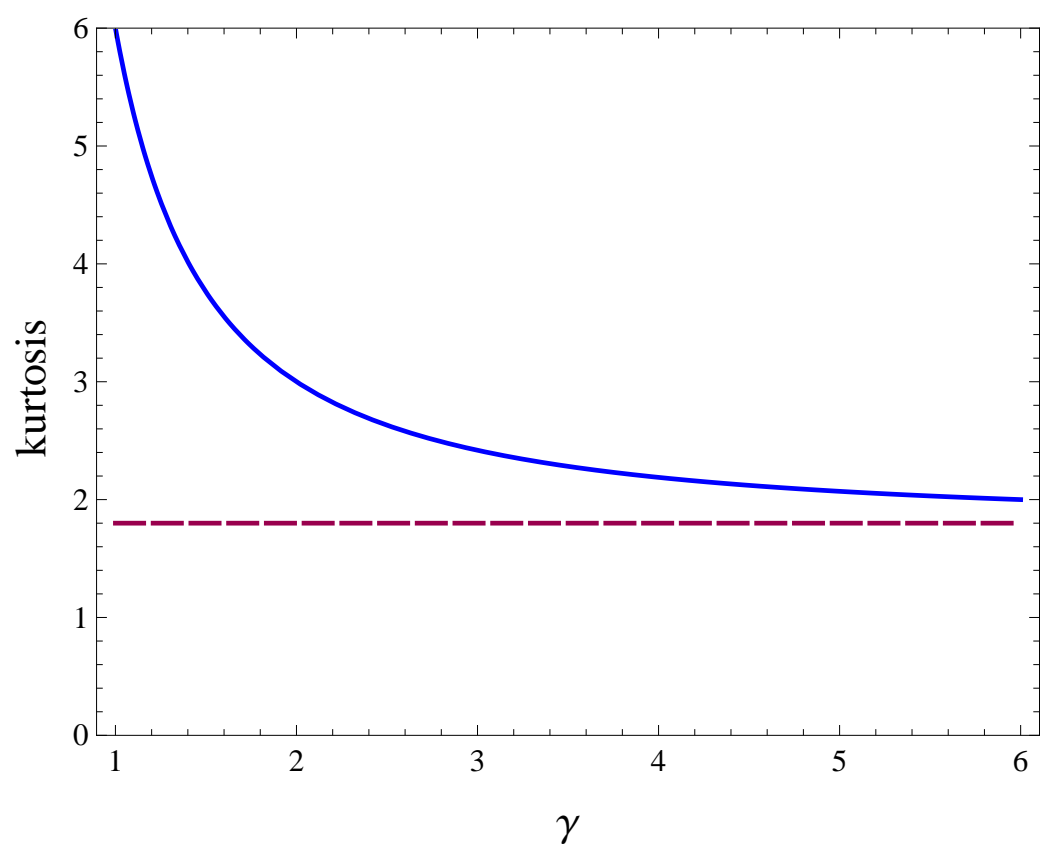

(a) kurtosis

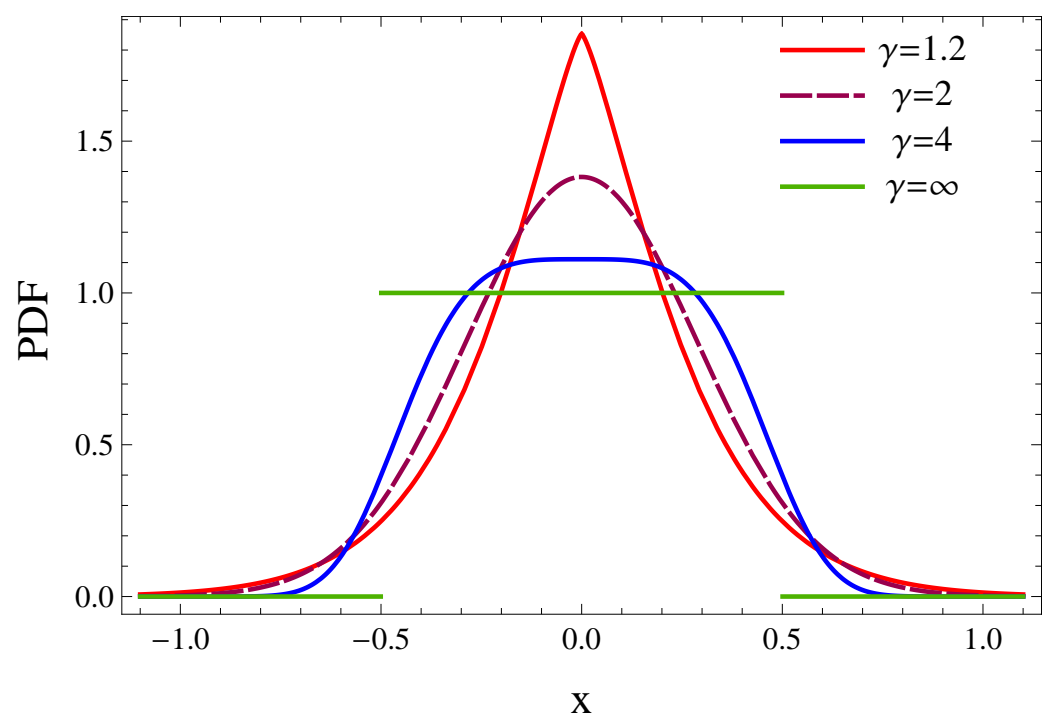

(b) probability density functions

Figure 3.10: A plot of the kurtosis vs. $\gamma$ and probability density functions for several values of $\gamma$. The dashed line $y=1.8$ in sub-figure (a) is the kurtosis of the uniform distribution.

In Figures 3.11-3.14 we create region plots showing where in parameter space the algebraic system in Thm. 3.1 has solutions. We show again that equilibriums exist on 
a set of full measure in parameter space for $\gamma=1.2,1.5,3,5$. One can reasonable infer that they exists for all $\gamma>1$. To determine if solutions to the algebraic system exist, we use a numerical method similar to that used for the Gaussian distribution in section 3.0.2. In Sub-Figure (c) of Figures 3.11-3.14 we see that the larger $n_{2}=2.5$ generally favors smaller values of $n_{1}$ and $n_{0}$ then those of $n_{2}=2.1$.

In Figure 3.15 through 3.17 we compare the regions where equilibria exist for successive values of $\gamma$. For instance, in Figure 3.15 we see the existence region for $\gamma=1.2$ is contained within the region for $\gamma=1.5$. This trend continues for larger values of $\gamma$, as is shown in Figure 3.16 and 3.17. This suggests that lower kurtosis kernels favor the formation of equilibrium solutions. 


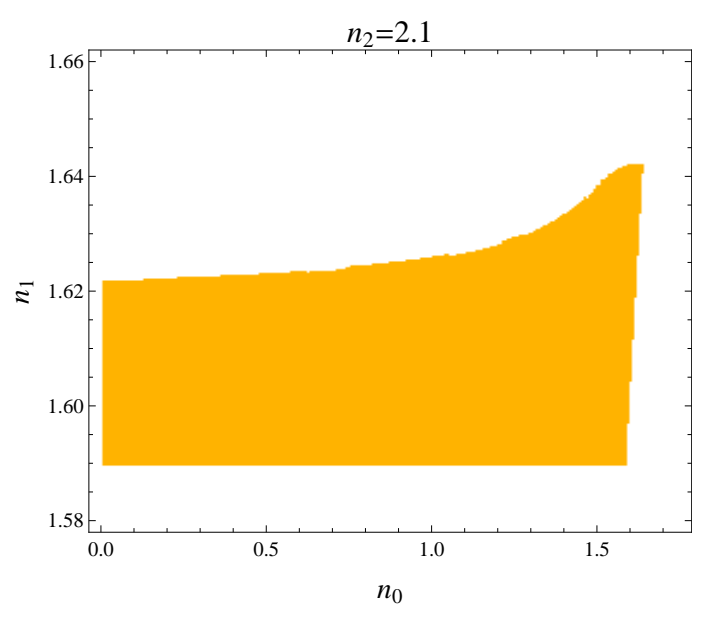

(a) $n_{2}=2.1$

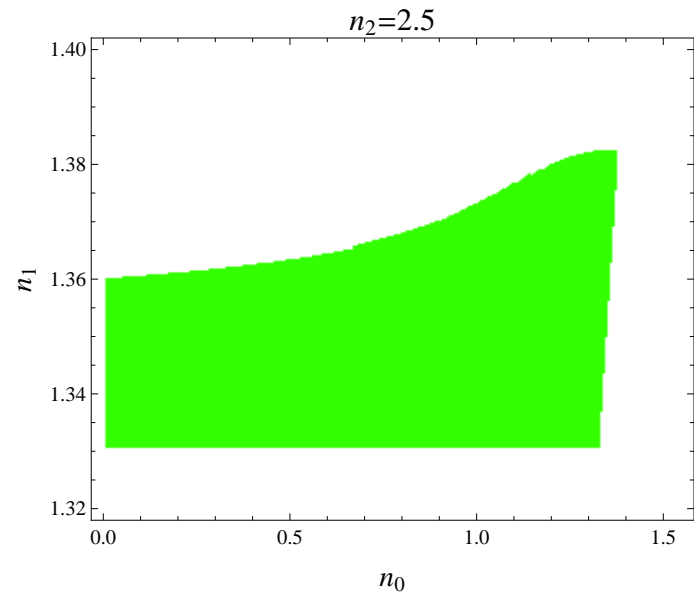

(b) $n_{2}=2.5$

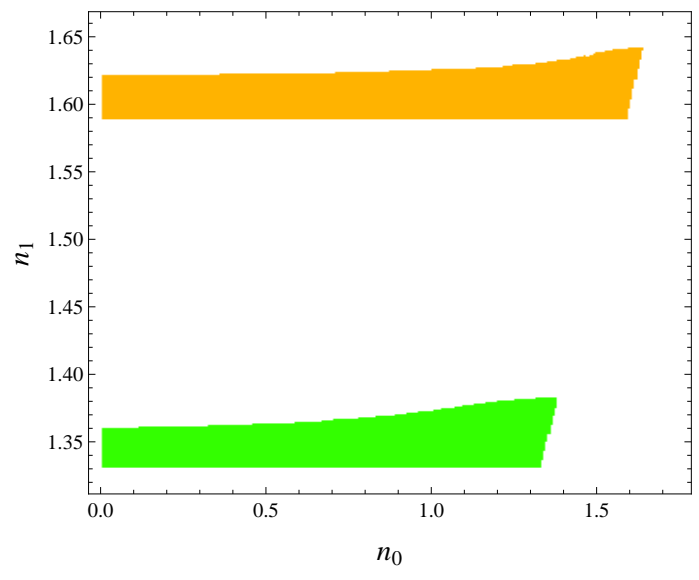

(c) $n_{2}=2.1$ and $n_{2}=2.5$ combined

Figure 3.11: Plot of the parameter regions where equilibriums exist when $k$ is an exponential power distribution with $\gamma=1.2$. Note differing scales in sub-plot (a) and (b). 


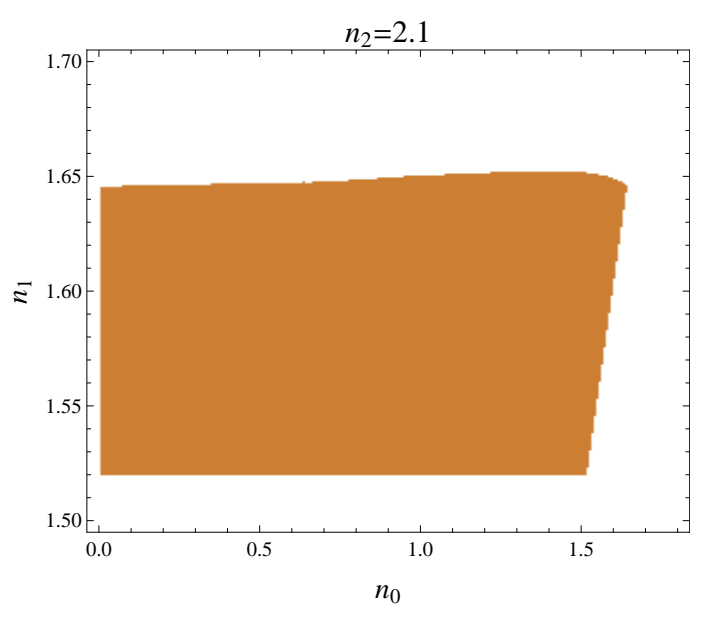

(a) $n_{2}=2.1$

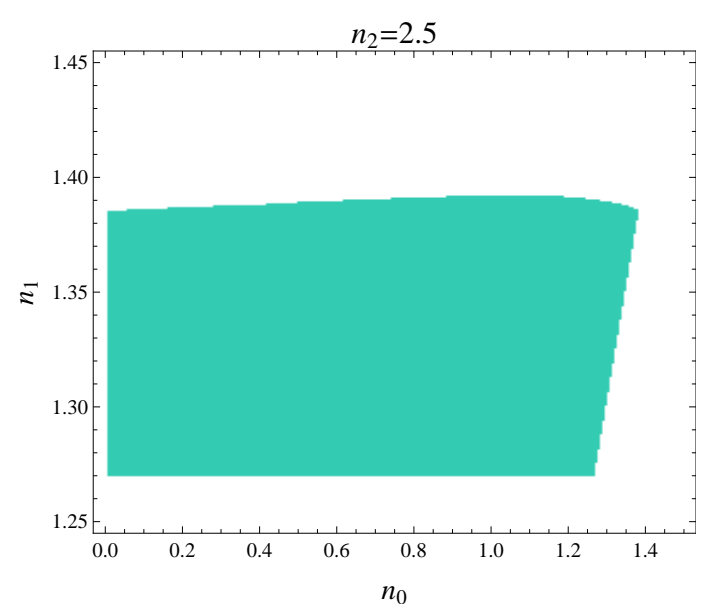

(b) $n_{2}=2.5$

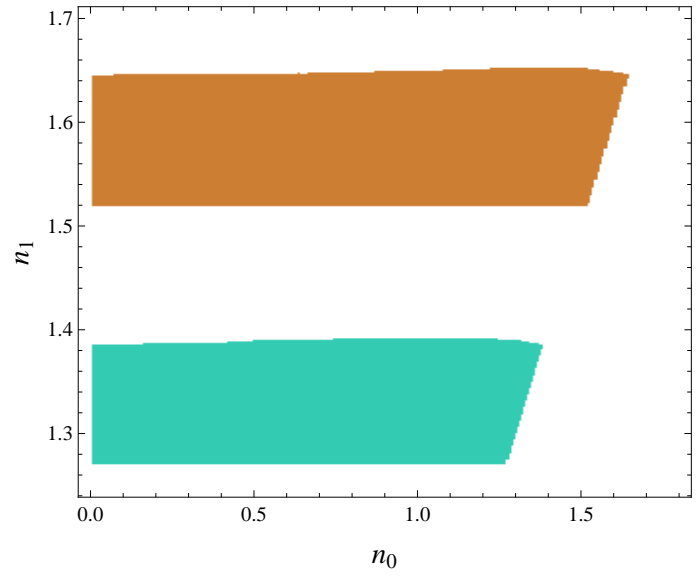

(c) $n_{2}=2.1$ and $n_{2}=2.5$ combined

Figure 3.12: Plot of the parameter regions where equilibriums exist when $k$ is an exponential power distribution with $\gamma=1.5$. Note differing scales in sub-plot (a) and (b). 


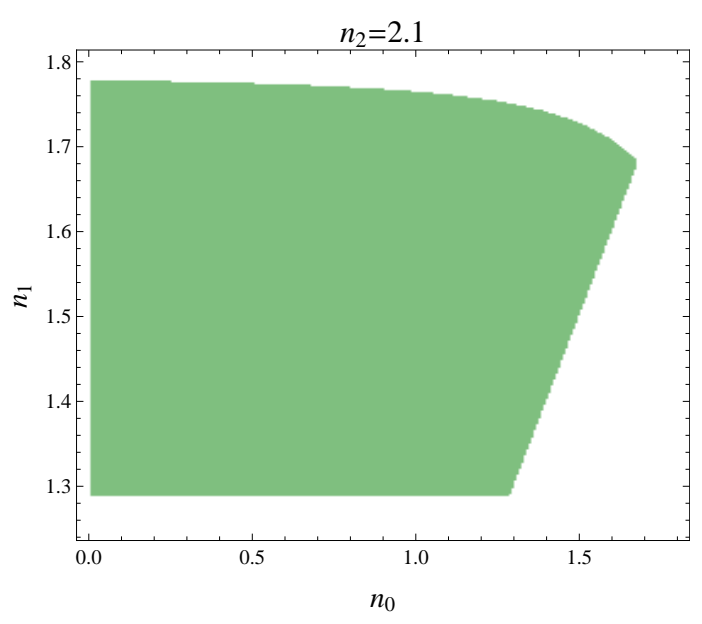

(a) $n_{2}=2.1$

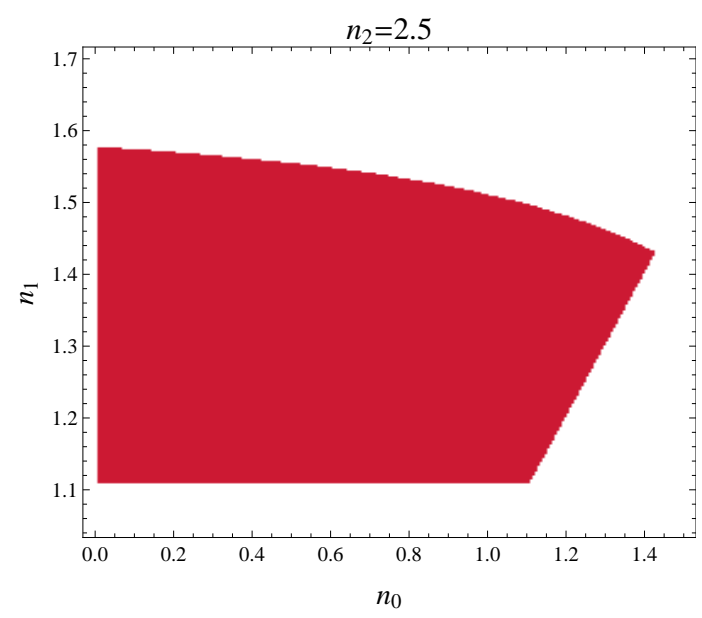

(b) $n_{2}=2.5$

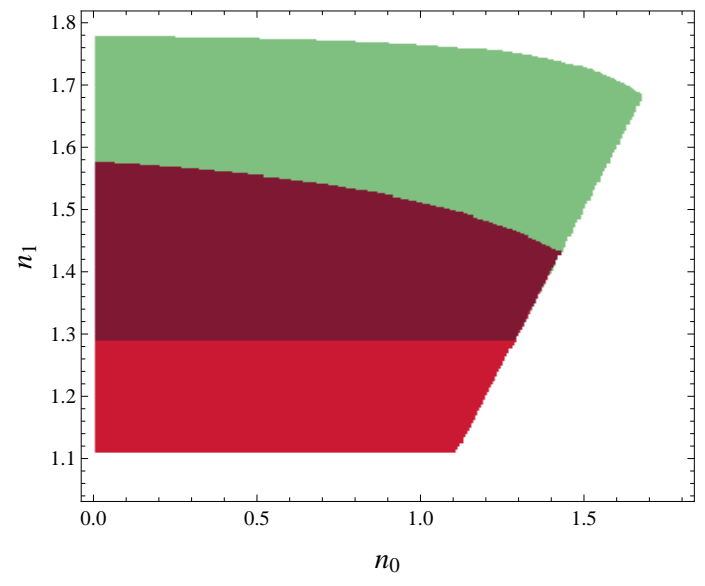

(c) $n_{2}=2.1$ and $n_{2}=2.5$ combined

Figure 3.13: Plot of the parameter regions where equilibriums exist when $k$ is an exponential power distribution with $\gamma=3$. Note differing scales in sub-plot (a) and (b). 


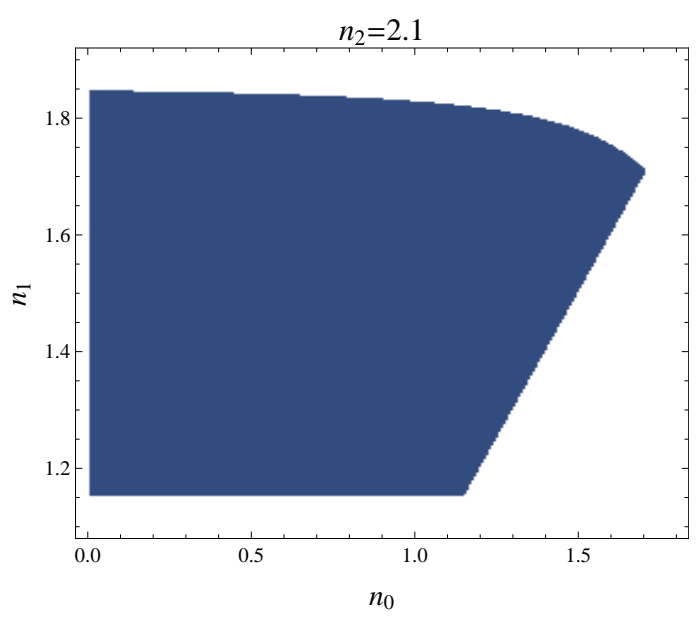

(a) $n_{2}=2.1$

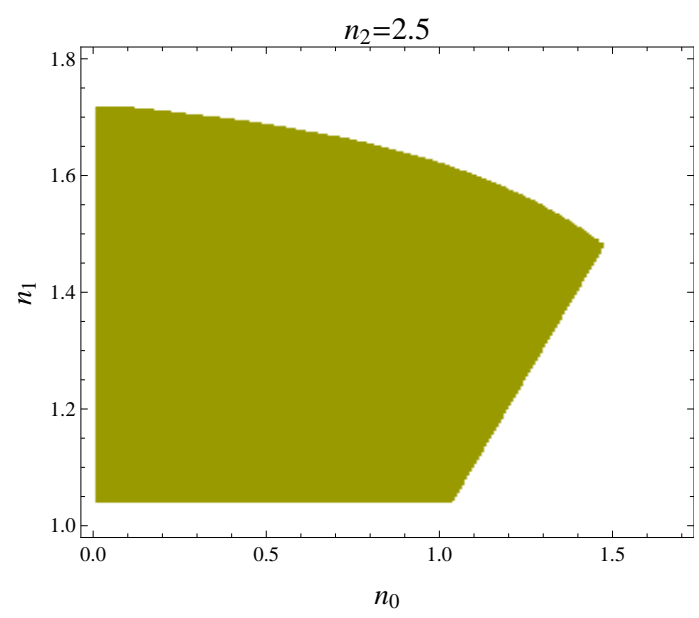

(b) $n_{2}=2.5$

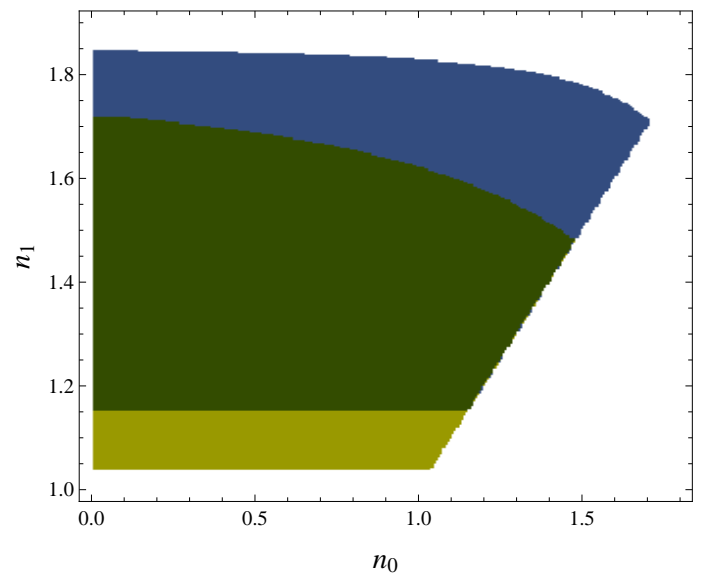

(c) $n_{2}=2.1$ and $n_{2}=2.5$ combined

Figure 3.14: Plot of the parameter regions where equilibriums exist when $k$ is an exponential power distribution with $\gamma=5$. Note differing scales in sub-plot (a) and (b). 


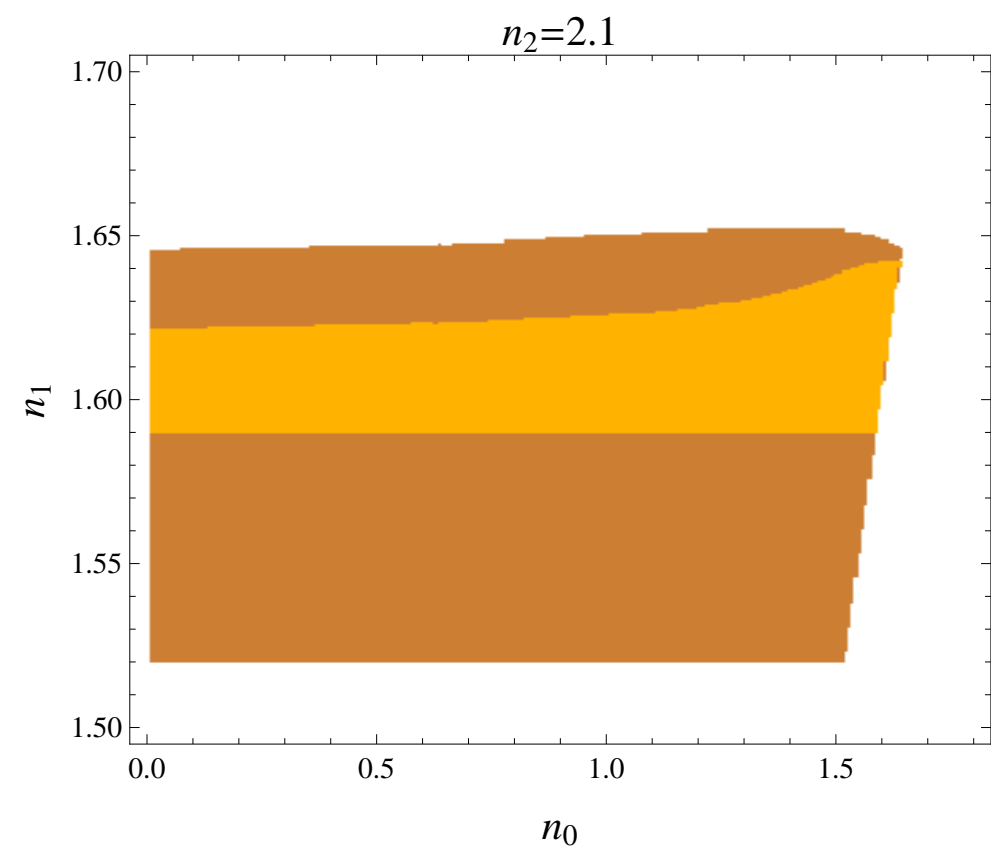

(a) yellow region is $\gamma=1.2$, brown region is $\gamma=1.5, n_{2}=2.1$

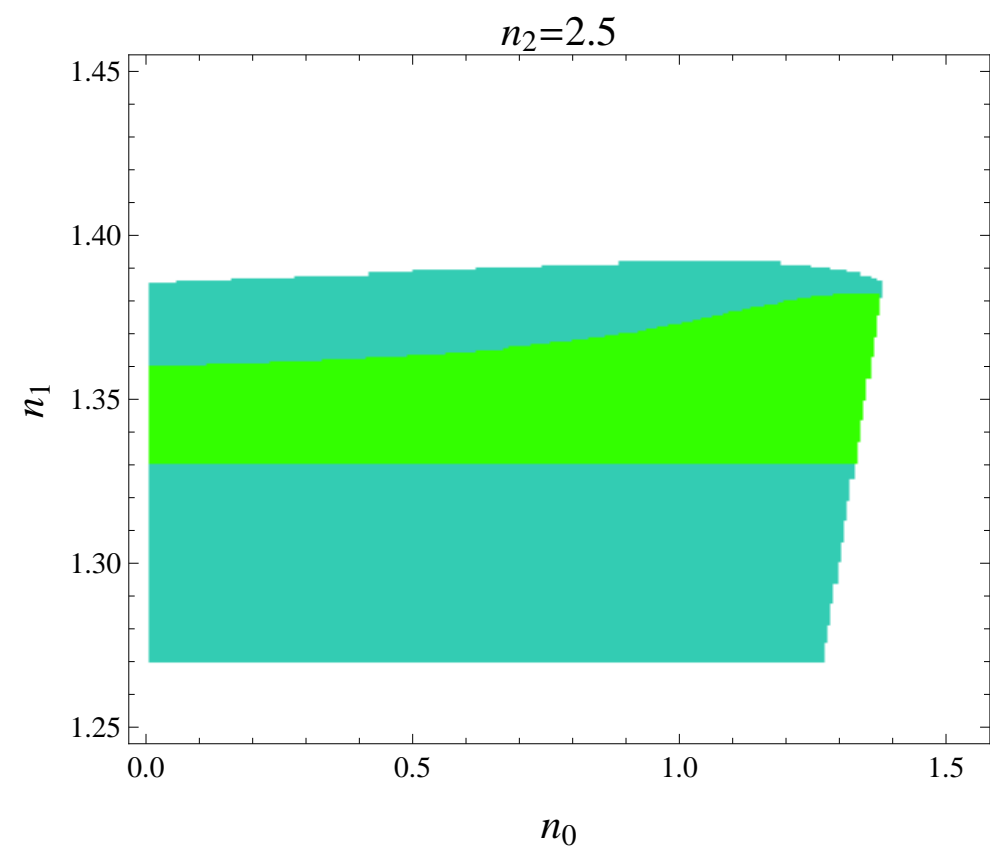

(b) green region is $\gamma=1.2$, turquoise region is $\gamma=1.5, n_{2}=2.5$

Figure 3.15: A comparison of the parameter values producing equilibrium for $\gamma=1.2$ to $\gamma=1.5$. Note the region for $\gamma=1.2$ is a proper subset of that for $\gamma=1.5$ for both values of $n_{2}$ depicted. 


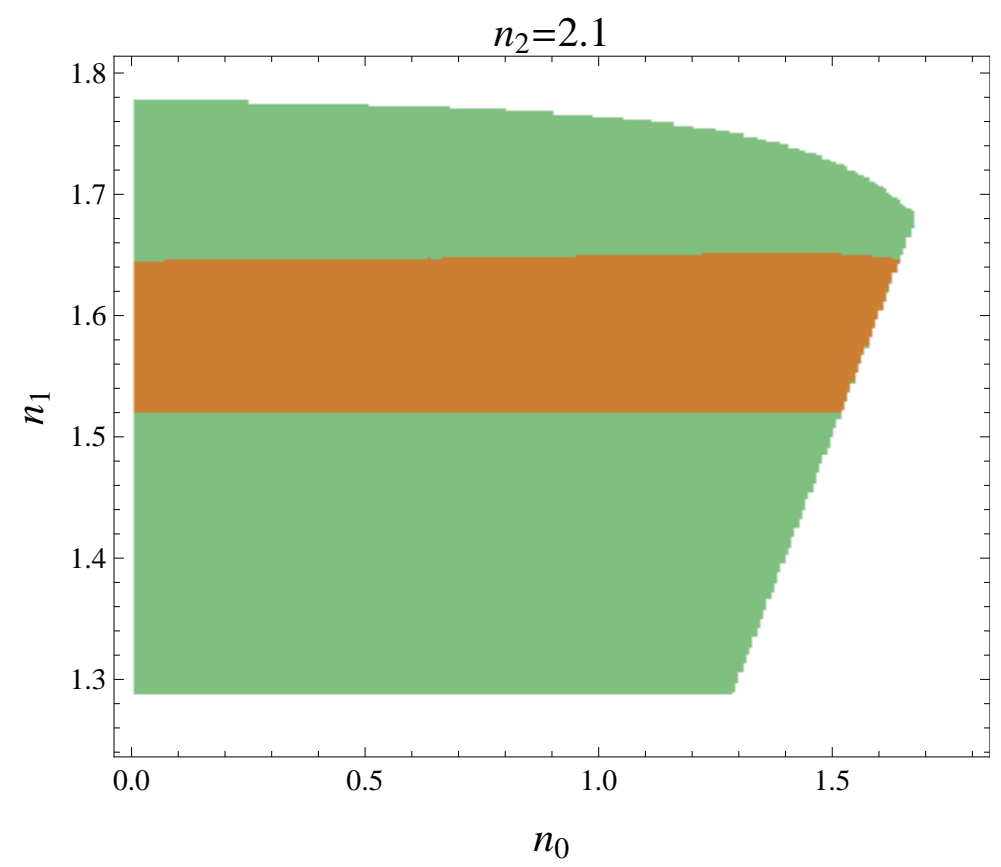

(a) green region is $\gamma=1.5$, brown region is $\gamma=3, n_{2}=2.1$

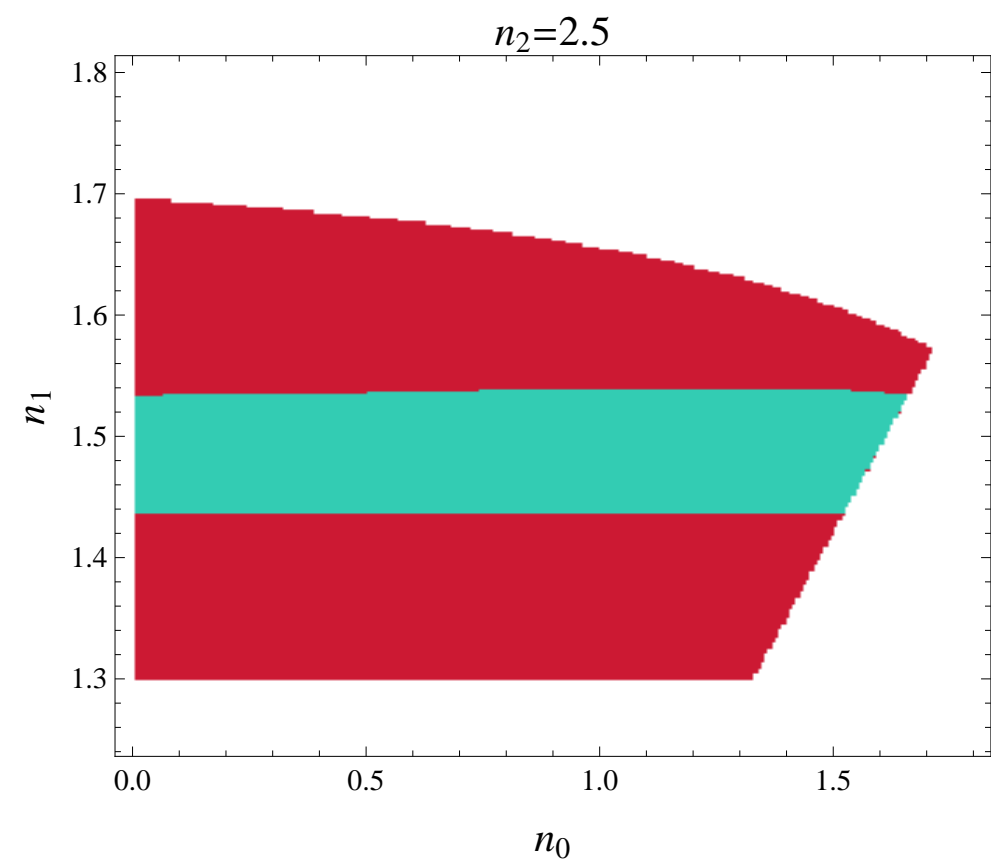

(b) turquoise region is $\gamma=1.2$, red region is $\gamma=3, n_{2}=2.5$

Figure 3.16: A comparison of the parameter values producing equilibrium for $\gamma=1.5$ to $\gamma=3$. Note the region for $\gamma=1.5$ is a proper subset of that for $\gamma=3$ for both values of $n_{2}$ depicted. 


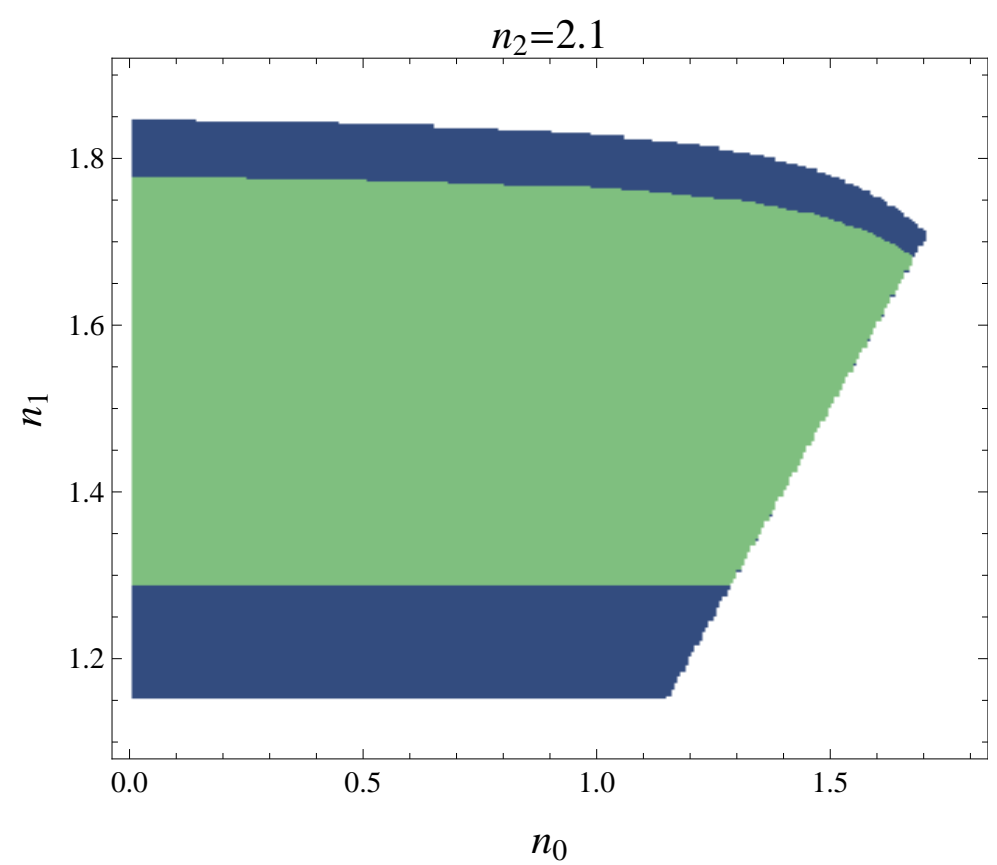

(a) green region is $\gamma=3$, blue region is $\gamma=5, n_{2}=2.1$

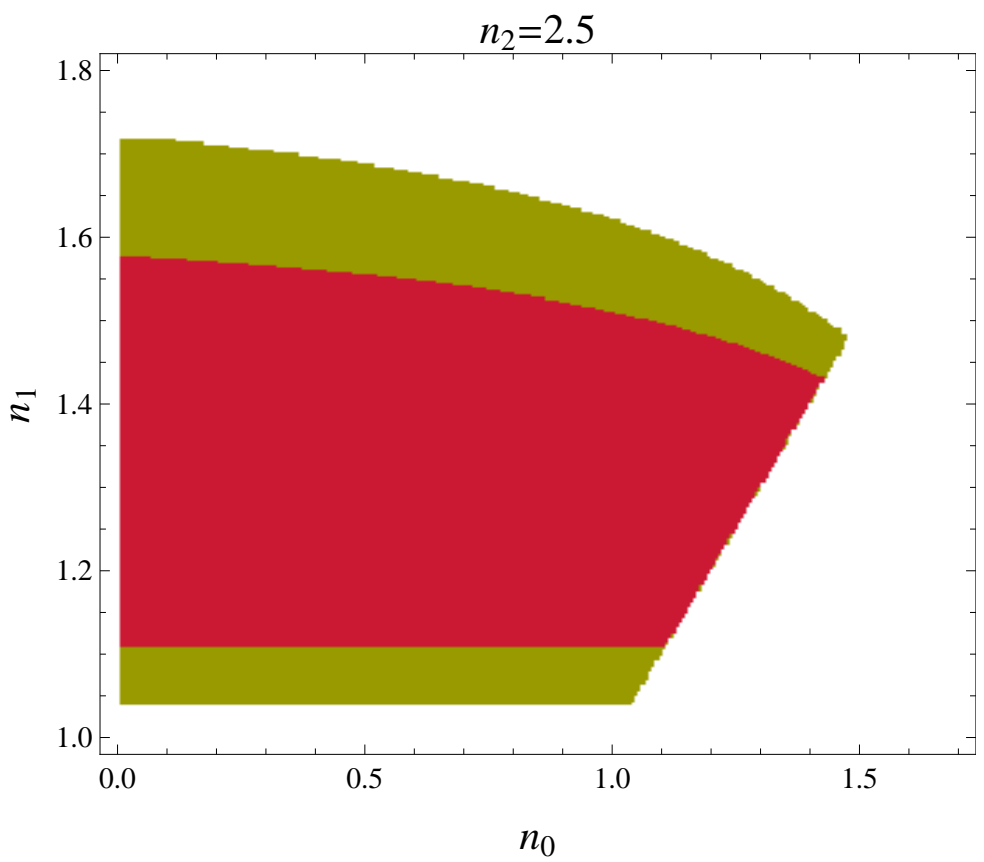

(b) red region is $\gamma=3$, gold region is $\gamma=5, n_{2}=2.5$

Figure 3.17: A comparison of the parameter values producing equilibrium for $\gamma=3$ to $\gamma=5$. Note the region for $\gamma=3$ is a proper subset of that for $\gamma=5$ for both values of $n_{2}$ depicted. 


\section{CHAPTER 4 \\ NUMERICAL RESULTS}

\subsubsection{Uniform dispersal with piecewise constant fecundity function}

4.0.4.1 Perturbed equilibria In Section 3.1 we demonstrated the existence of non-spreading equilibrium solutions for the uniform kernel with the fecundity function defined in Eq. 3.2. In this section we will examine the behavior of perturbed equilibria. The iterates of Model 2.1 are particularly easy to numerically compute for these definitions of $k$ and $g$. If $u_{0}$ is a piecewise defined linear spline, then the intervals of $x$ where $\left\{x \mid 0<u_{0}(x)<1\right\},\left\{x \mid 1<u_{0}(x)<n_{1}\right\}$, and $\left\{x \mid n_{1}<u_{0}(x)\right\}$ can be determined, which

in turn determines $g\left(u_{0}(x)\right)$. The convolution with $k(x)$ can then be exactly determined, thus giving $u_{1}(x)$.

To choose a parametric form of perturbation for $u_{e}$, Eq. 3.2 provides us a suggestive form. Namely by shifting the discontinuity points of $G_{\{a, b\}}$ from $\{-b,-a, a, b\}$ to the respectively nearby points $\left\{-b_{1},-a_{1}, a_{2}, b_{2}\right\}$ and then taking the convolution with $k$, we can create a four parameter perturbed equilibrium that converges to $u_{e}$ as $b_{1}, b_{2} \rightarrow b$ and $a_{1}, a_{2} \rightarrow a$. This form is fairly general, in that it represents the first iterate of any uni-modal initial data that exceeds $n_{1}$ at its maximum.

We define the perturbed initial state as

$$
\tilde{u}_{e}=k * \widetilde{G}_{\left\{-b_{1},-a_{1}, a_{2}, b_{2}\right\}},
$$


where

$$
\widetilde{G}_{\left\{-b_{1},-a_{1}, a_{2}, b_{2}\right\}}(x)= \begin{cases}n_{2}, & -b_{1}<x<-a_{1} \\ n_{0}, & -a_{1}<x<a_{2} \\ n_{2}, & a_{2}<x<b_{2} \\ 0, & \text { otherwise. }\end{cases}
$$

Obviously the parameters must conform to the inequality $-b_{1}<-a_{1}<a_{2}<b_{2}$, and so as to neglect trivial translations we choose $\left\{-b_{1},-a_{1}, a_{2}, b_{2}\right\}$ so as to fix the center of mass of $\widetilde{G}_{\left\{-b_{1},-a_{1}, a_{2}, b_{2}\right\}}(x)$ at $x=0$.

To study the stability of the equilibriums it is helpful to use a domain-size versus time plot. For our purposes we define the domain size of $u_{i}(x)$ to be the length of the support of $u_{i}(x)$ in the case that the support is a single interval, or more generally as

$$
\sup \left\{x \mid u_{i}(x)>0\right\}-\inf \left\{x \mid u_{i}(x)>0\right\}
$$

For instance, if the domain size is linearly increasing with time, this indicates the perturbed solution has converged to a spreading solution. If the domain size oscillates with a period $p$, this is a strong indication that the perturbed solution has converged to a period- $p$ attractor.

We observe a wide variety of behaviors. For a fixed $n_{2}$, if weaker growth parameters are chosen for $n_{1}$ and $n_{0}$, then perturbations to the equilibrium can lead to extinction. This is observed in Figure 4.1. For a fixed $n_{2}$, if strong growth parameters are chosen for $n_{1}$ and $n_{0}$, then perturbations to the equilibrium can lead to spreading solutions, as is observed in Figure 4.2. However for intermediate values of $n_{1}$ and $n_{0}$ a wide variety of stable non-spreading periodic solutions can be found. For example in Figures 4.3-4.5, the same set of parameters can lead either to a non-spreading period-5 orbit, or a nonspreading period-105 orbit depending on the initial perturbation. A wide a variety of short and long periodicities can be observed for other parameter values. 


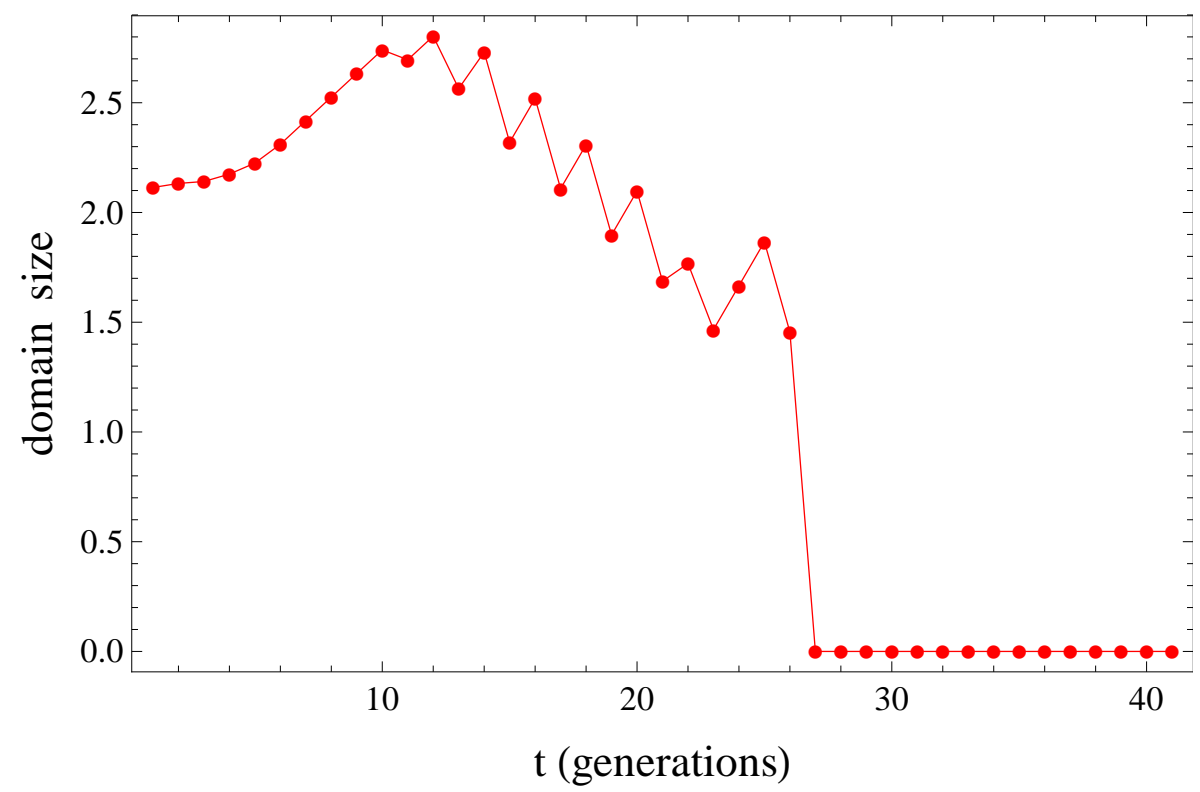

Figure 4.1: A domain-size vs. time plot of an equilibrium where perturbation leads to extinction. $\left\{n_{0}, n_{1}, n_{2}\right\}=\{1.1,1.4,2.5\}$. The equilibrium is $k * G_{\{0.2334,0.5548\}}$, and the perturbed initial state is $k * \widetilde{G}_{\{-0.5553,-0.2212,0.2353,0.5586\}}$. 


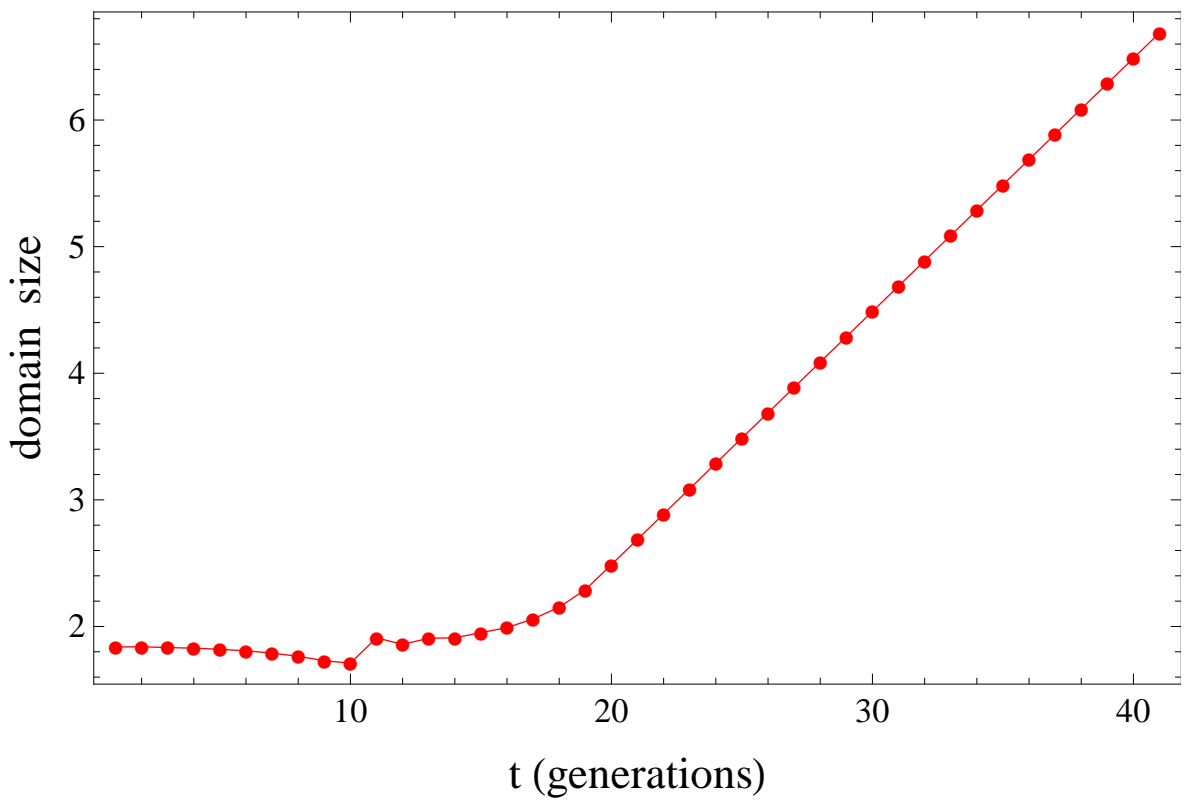

(a) domain-size vs. time

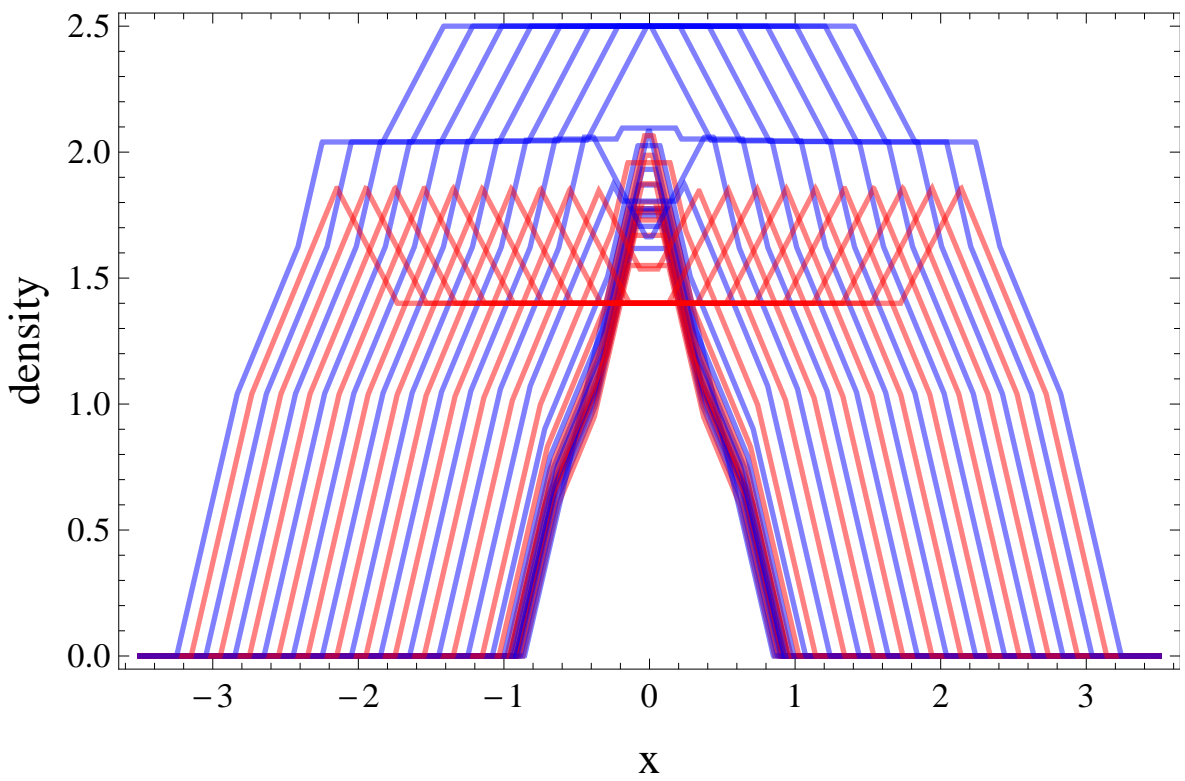

(b) Plot of $u_{1}(x)$ through $u_{40}(x)$ vs. $x$. Red denotes odd years, blue denotes even years.

Figure 4.2: A case where perturbations lead to spreading solutions. $\left\{n_{0}, n_{1}, n_{2}\right\}=$ $\{1.4,1.6,2.5\}$. The equilibrium is $k * G_{\{0.1508,0.4236\}}$, and the perturbed initial state is $k * \widetilde{G}_{\{-0.4189,-0.1439,0.1471,0.4193\}}$. 


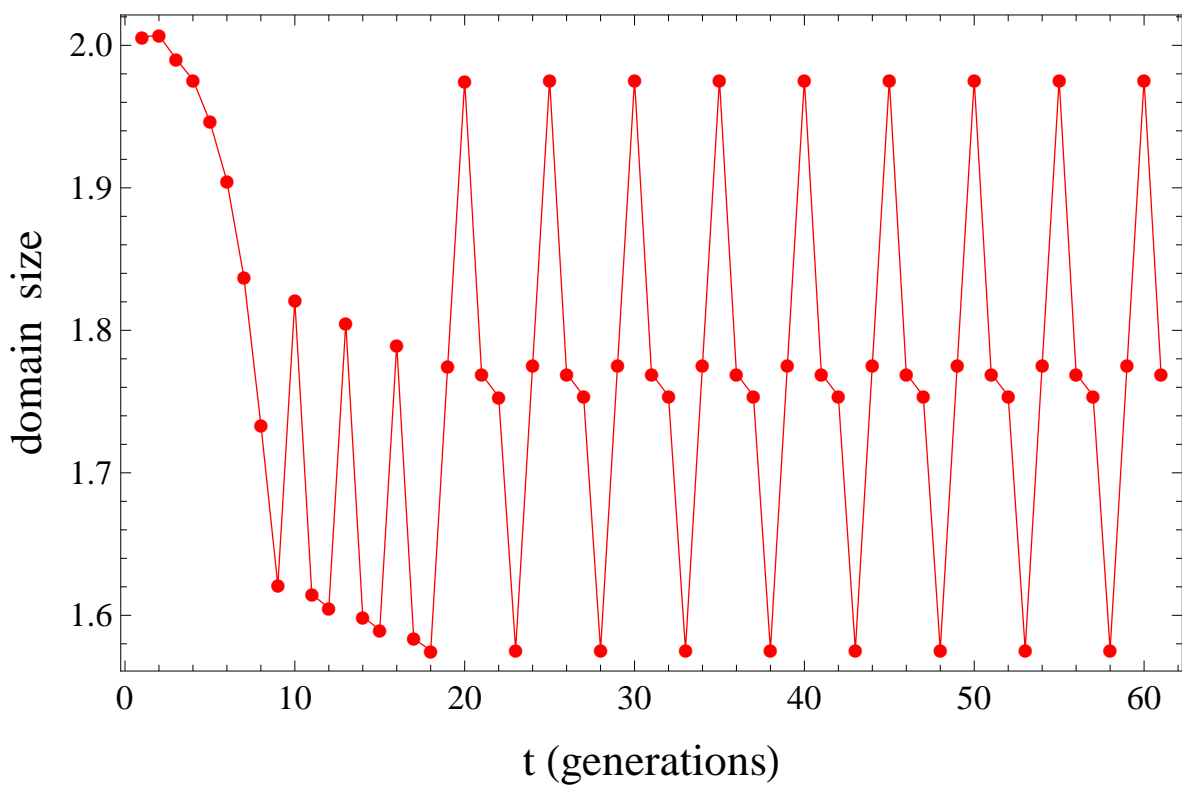

Figure 4.3: A domain-size vs. time plot for a perturbation leading to a period-5 orbit. $\left\{n_{0}, n_{1}, n_{2}\right\}=\{1.3,1.45,2.5\}$. The equilibrium is $k * G_{\{0.2205,0.5122\}}$, and the perturbed initial state is $k * \widetilde{G}_{\{-0.5024,-0.2078,0.2134,0.5035\}}$. 


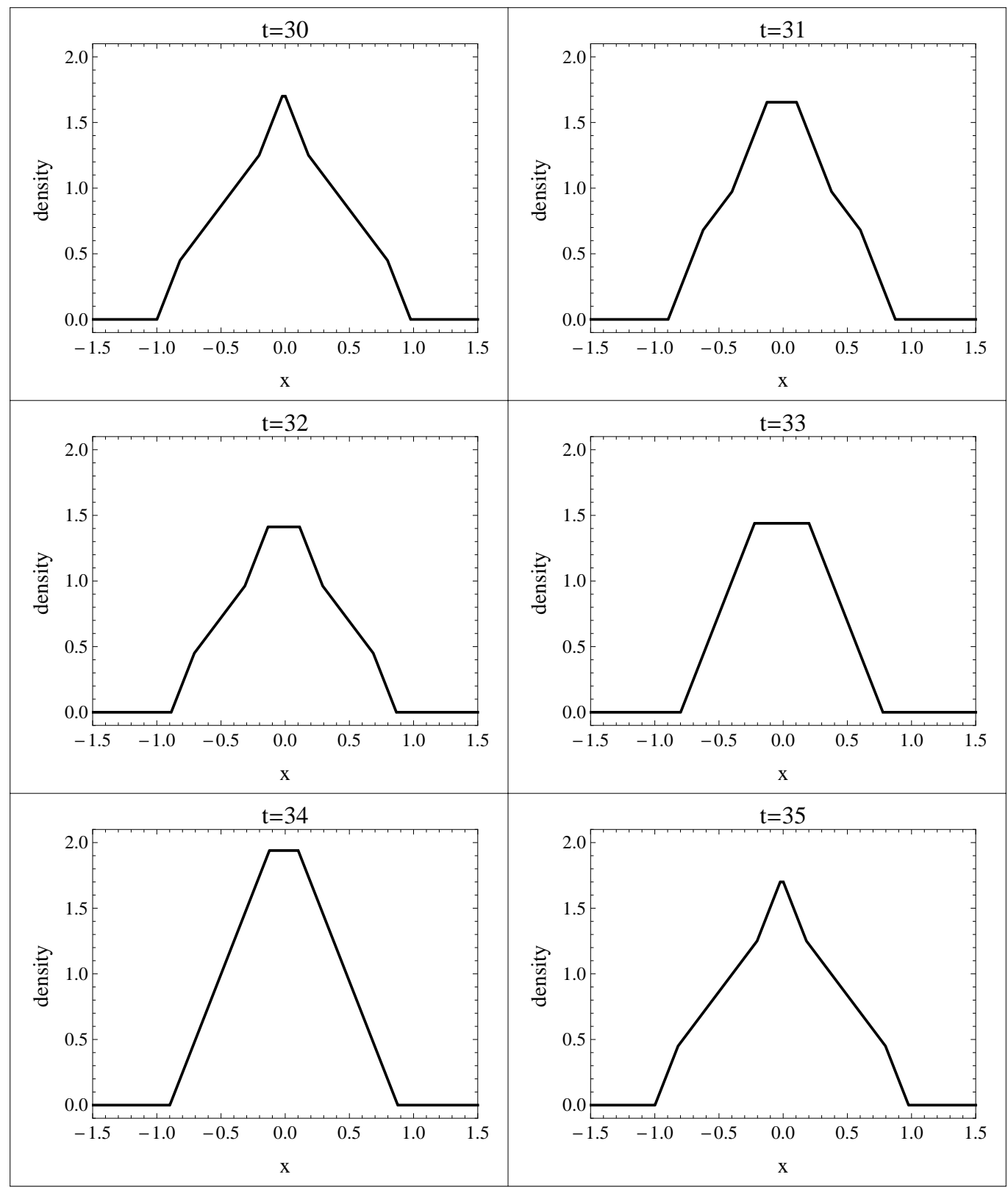

Figure 4.4: A plot of $u_{30}(x)$ through $u_{35}(x)$ showing a complete period. The parameters and initial condition are the same as in Figure 4.3. 


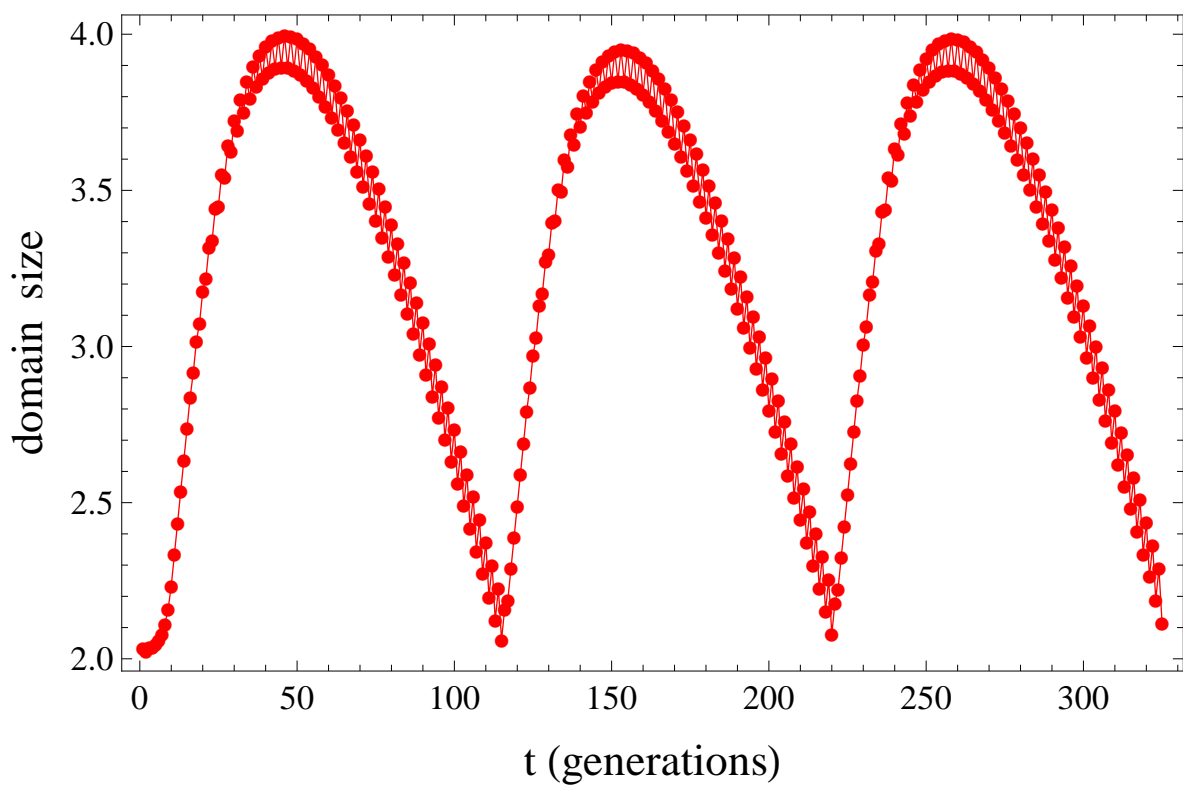

(a) domain-size vs. time

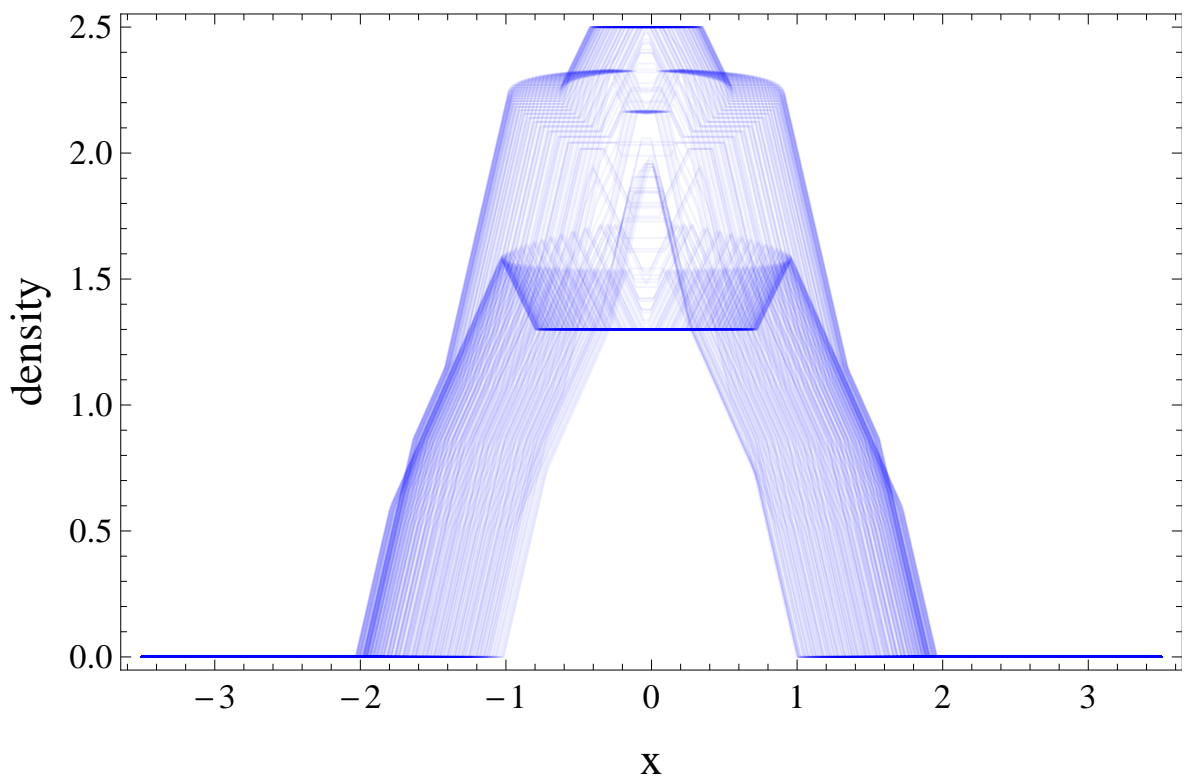

(b) Plot of $u_{1}(x)$ through $u_{325}(x)$. Curves are rendered partially transparent so as to show points of accumulation.

Figure 4.5: A case where the same parameters used in Figure 4.4 have a different orbit when a different initial condition is used. The periodicity of this orbit is 105 . The initial state is $k * \widetilde{G}_{\{-0.5160,-0.2232,0.2387,0.5193\}}$. 
4.0.4.2 Initial domain size It interesting to consider what initial domain size will lead to non-spreading solutions as opposed to extinction or spreading solutions. To gain some insight into this question we conduct simulations where the initial data is a Heaviside Pi (boxcar) function, where the half-width ,w, is treated as a free parameter. We thus let the initial data be

$$
u_{0}(x)=h_{w}(x):= \begin{cases}n_{2}, & |x|<w \\ 0, & |x|>w\end{cases}
$$

We would expect that for $w$ sufficiently small, $Q\left[h_{w}\right](x)<1$ as $g\left(h_{w}(x)\right)$ will have insufficient mass to exceed the Allee threshold when convolved with $k$. In fact we can put a lower bound on the minimum survivable domain size by computing $Q\left[h_{w}\right](0)$ with the assumption $w<0.5$. Since $Q\left[h_{w}\right](x)$ will be symmetric and uni-modal $Q\left[h_{w}\right](0)$ will be a maximum. We find $Q\left[h_{w}\right](0)=2 n_{0} w$, therefore setting $Q\left[h_{w}\right](0)=1$ we find if $w<\frac{1}{2 n_{0}}$ the population will go extinct. In Figures 4.6 through 4.11 we plot the half-domain size as a function of $w$ at $t=88, t=100$, and $t=112$. The domain size is defined the same as in Section 4.0.4.1. The $t=88$ data points are rendered in red, $t=100$ are rendered in green, and $t=112$ are rendered in blue.

In the simplest case for a set of parameters where spreading solutions are possible, we might expect that there would be an interval $\left(0, w_{1}\right)$ where extinction occurs, followed by the interval $\left(w_{1}, w_{2}\right)$ where non-spreading solutions occur, and then finally on $\left(w_{2}, \infty\right)$ we would expect spreading solutions to occur. Indeed in Figure 4.6 we find such a case, where on the interval $0.36<w<0.64$ the tight clustering of the respective generations indicates non-spread. For $w>0.64$ we see the respective generations are well separated by a positive distance indicating spread. In sub-figure (b), as evidence that solutions for $0.36<w<0.64$ are non-spreading, we demonstrate with a domain size vs. time plot that the solution for $w=0.5$ settles into a long period non-spreading solution. Similarly, in sub-figure (c) we demonstrate that for $w>0.64$ solutions evolve into spreading solutions 
by way of a domain size vs. time plot for $w=0.75$.

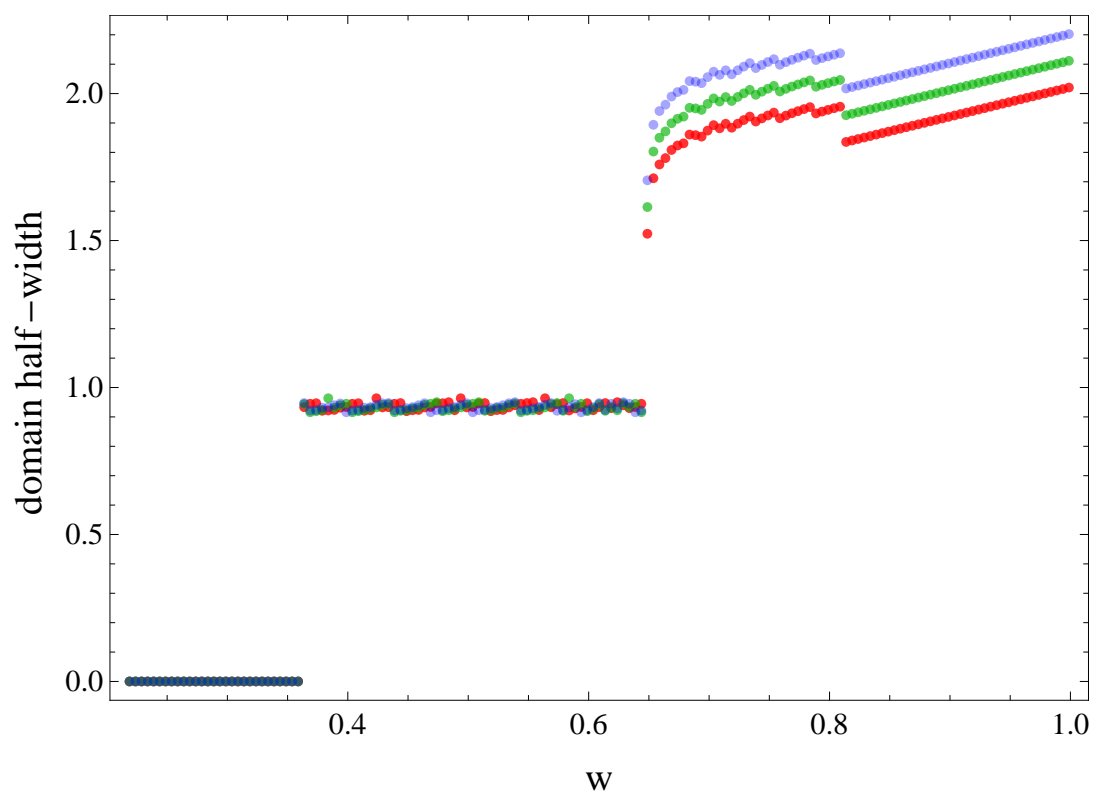

(a)

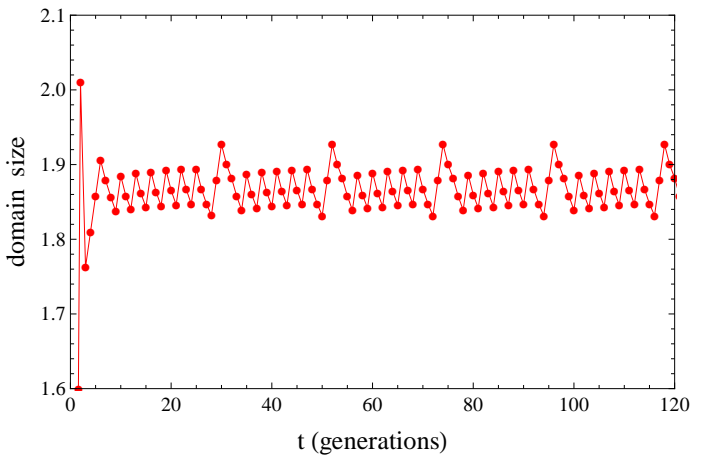

(b) domain vs. $t$ for $w=0.5$

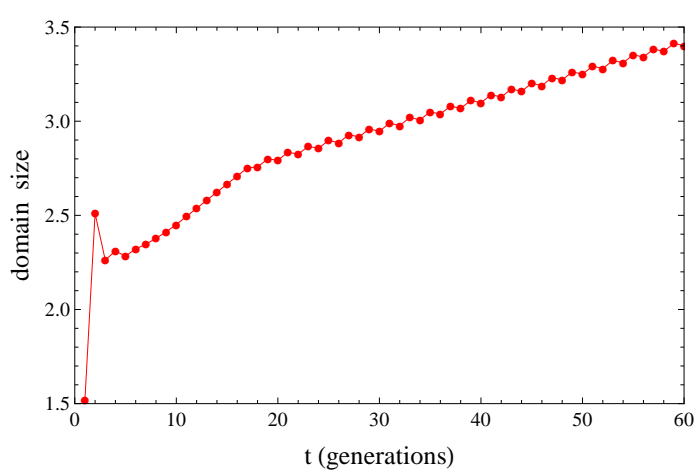

(c) domain vs. $t$ for $w=0.75$

Figure 4.6: Domain size after 88 iterations (red), 100 iterations (green), and 112 iterations (blue) as a function of initial domain half-width. In sub-figure (b) a the domain vs. time plot is shown for $w=0.5$. In sub-figure (c) the domain vs time plot is shown for $w=0.75$. Parameter values used are $\left(n_{0}, n_{1}, n_{2}\right)=(1.6,1.75,2.1)$.

We see in Figure 4.7 we see it largely follows the pattern of Figure 4.6, with nonspreading solutions for most of $0.57<w<0.8$, however we see at $w=0.66$ and $w=0.76$ spreading solutions emerge, indicating more complex dynamics then with Figure 4.6. 


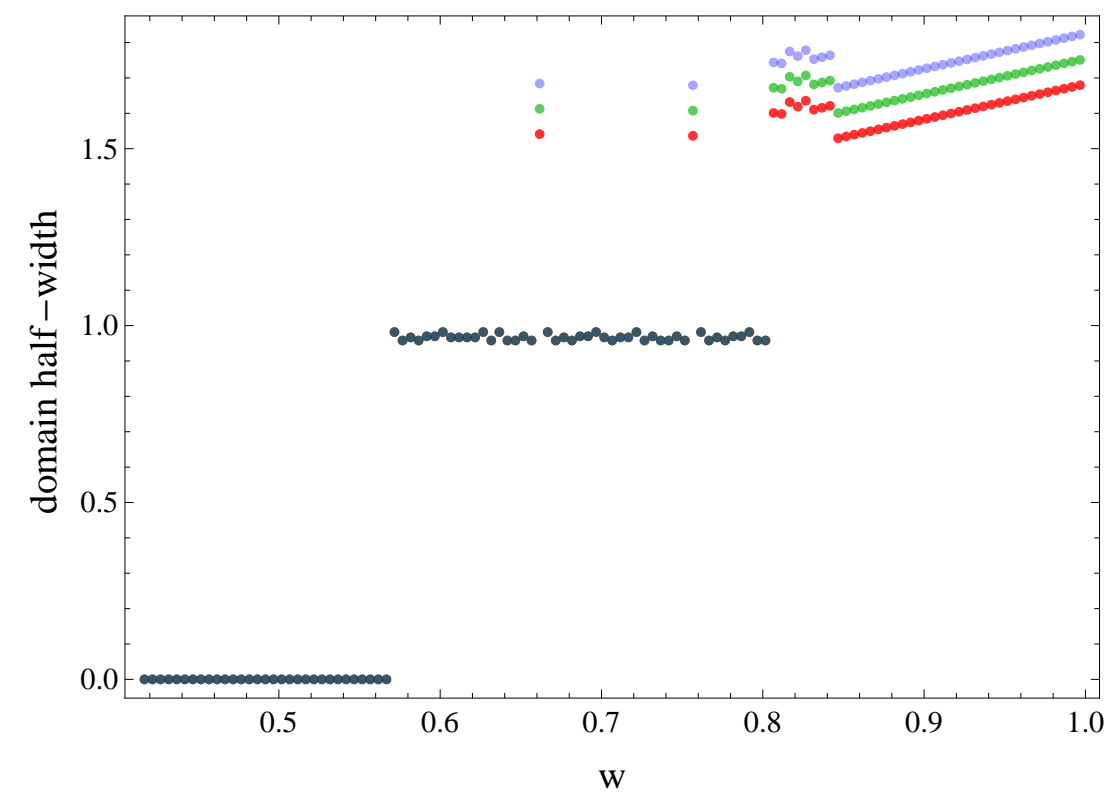

(a)

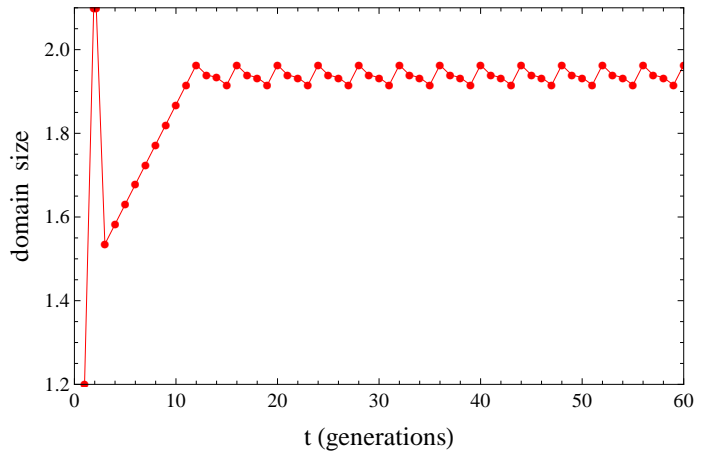

(b) domain vs. $t$ for $w=0.6$

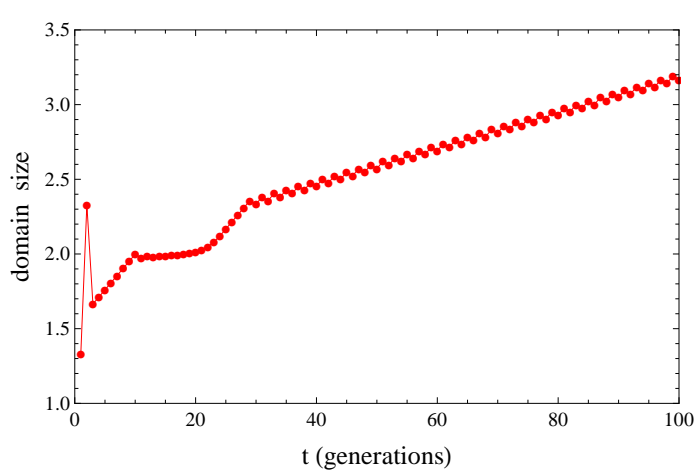

(c) domain vs. $t$ for $w=0.665$

Figure 4.7: Domain size after 88 iterations (red), 100 iterations (green), and 112 iterations (blue) as a function of initial domain half-width. In sub-figure (b) a the domain vs. time plot is shown for $w=0.6$. In sub-figure (c) the domain vs time plot is shown for $w=0.665$. Parameter values used are $\left(n_{0}, n_{1}, n_{2}\right)=(1.2,1.9,2.1)$.

In Figure 4.8 we see for all $w>0.57$ there are 5 distinct bands of domain sizes. This indicates regardless of initial domain size, the solution will converge to a nonspreading period 5 attractor. In Figure 4.8-b we show the domain-size vs. time for $w=1.1$ which demonstrates the solution settling into a period 5 orbit. While all values of $w>0.57$ eventually reach a period 5 orbit, we see by multi-coloration of the bands 
in Figure 4.8 that clearly the phase will be sensitive to $w$.

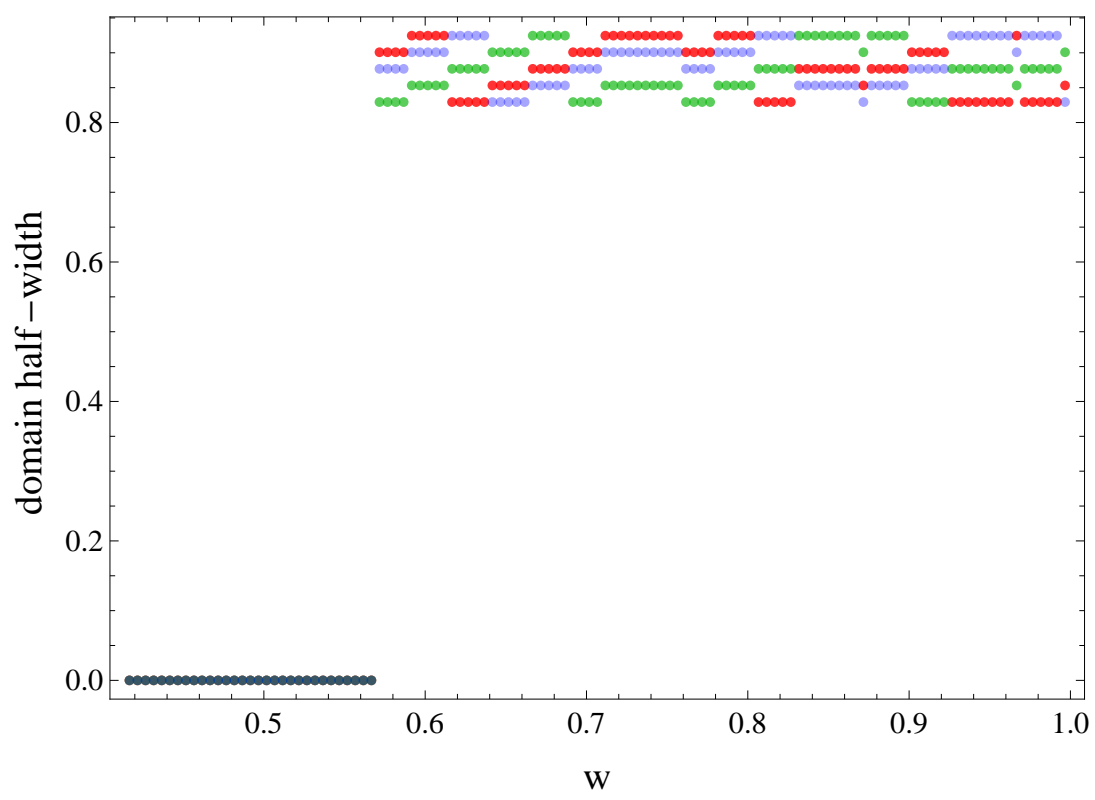

(a) domain size vs. $w$

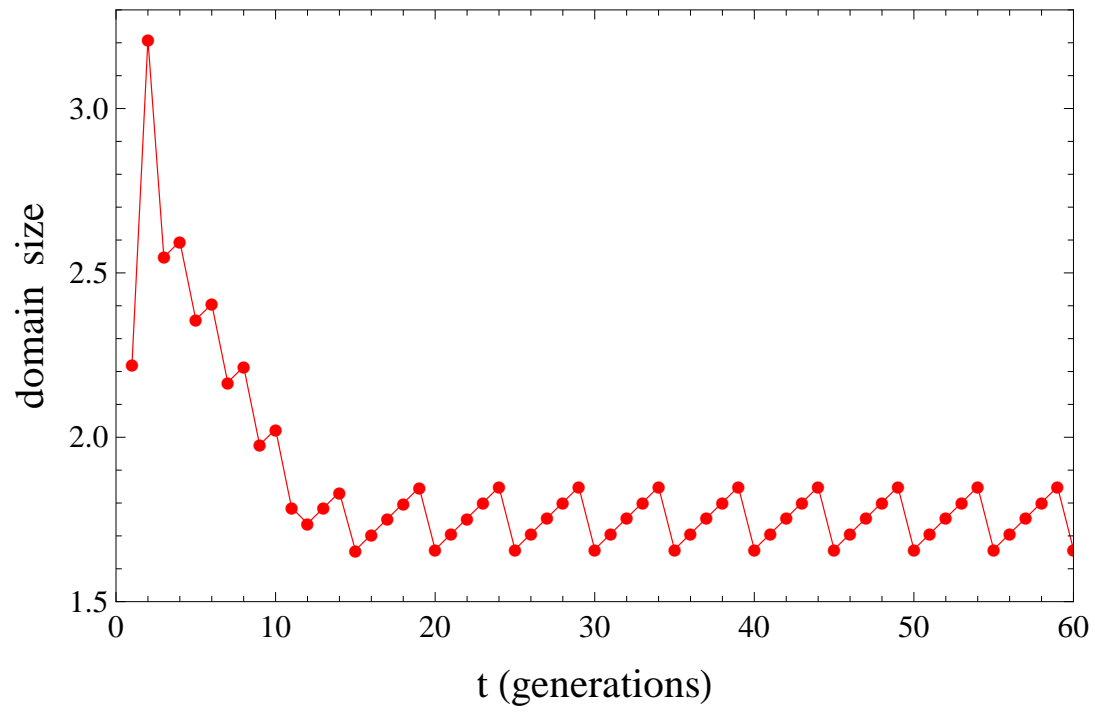

(b) domain size vs. $t$ for $w=1.1$

Figure 4.8: In sub-figure (a) domain size after 88 iterations (red), 100 iterations (green), and 112 iterations (blue) as a function of initial domain half-width. In sub-figure (b) domain size vs. $t$ for $w=0.6$ is depicted. Parameter values used are $\left(n_{0}, n_{1}, n_{2}\right)=$ $(1.2,1.6,2.1)$.

In Figure 4.9 we see the dependance on initial domain size becomes even more 
complex. At $w \approx 0.48$ we see there is an interval of spreading solutions between the extinction and non-spreading initial domain lengths. There are 2 intervals of non-spreading $w,(0.49,0.54)$, and $(0.59,0.64)$ which are disrupted by an interval of spreading solutions. Finally for $w>0.64$ we see the solutions are spreading.

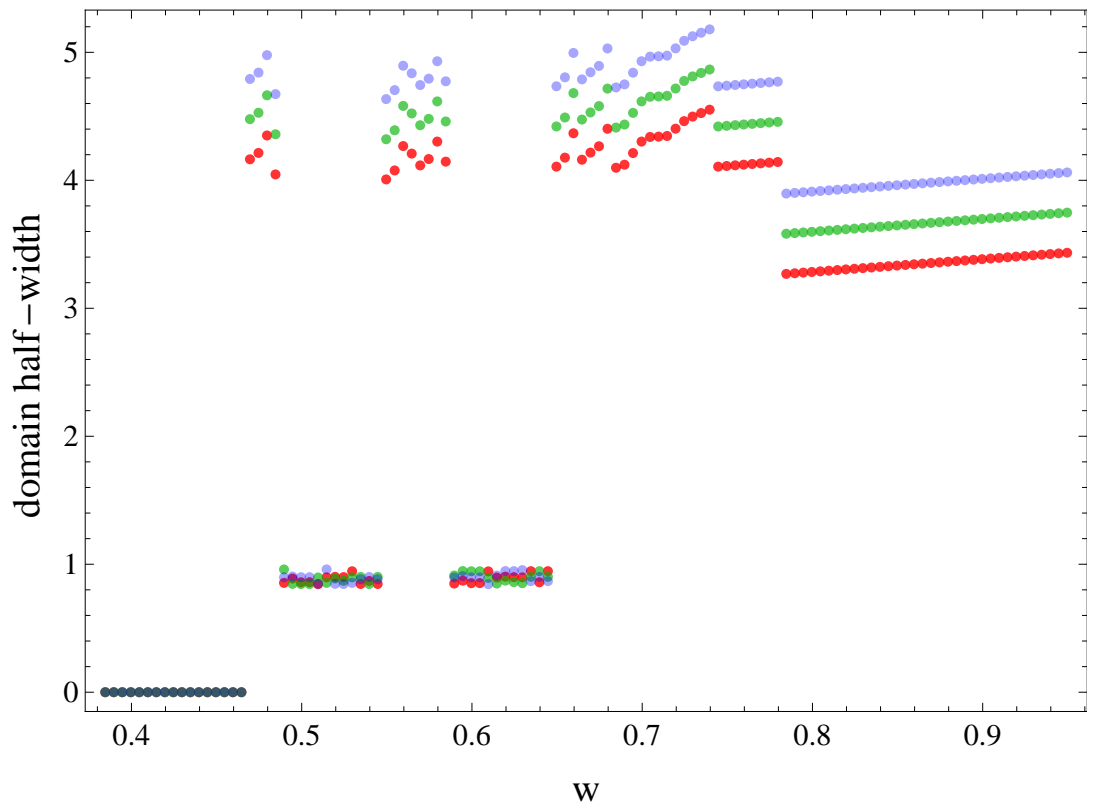

(a)

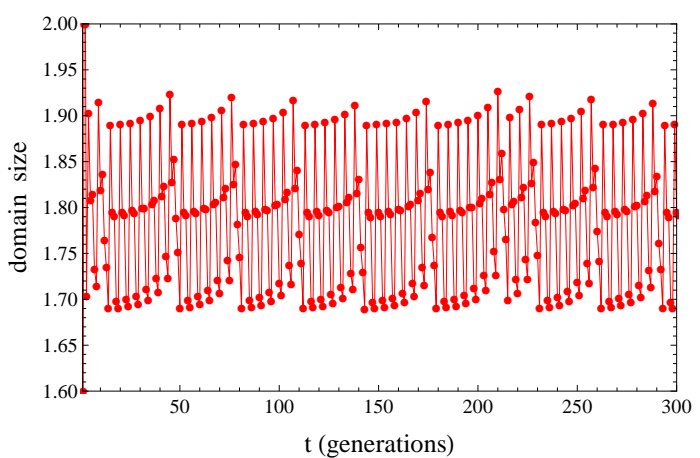

(b) domain vs. $t$ for $w=0.62$

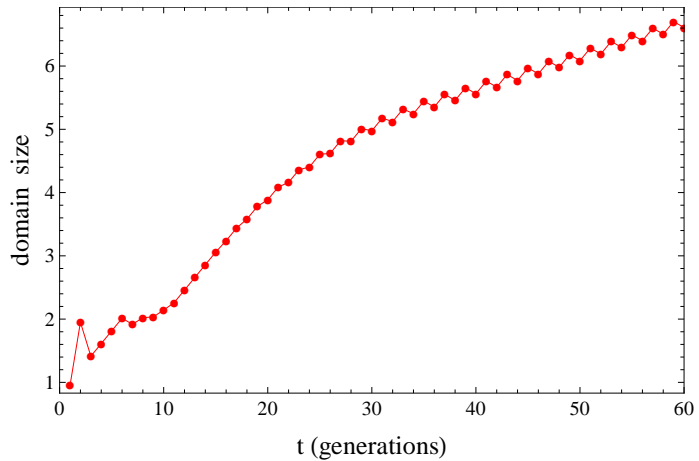

(c) domain vs. $t$ for $w=0.475$

Figure 4.9: Domain size after 88 iterations (red), 100 iterations (green), and 112 iterations (blue) as a function of initial domain half-width. In sub-figure (b) a the domain vs. time plot is shown for $w=0.62$. In sub-figure (c) the domain vs time plot is shown for $w=0.475$. Parameter values used are $\left(n_{0}, n_{1}, n_{2}\right)=(1.3,1.6,2.5)$. 
In Figure 4.10 we again see a complex dependency on initial domain size. We see on the interval $0.69<w<0.9$ there are non-spreading solutions interspersed with two intervals of spreading solutions. Unlike in Figure 4.9 we see at $w \approx 0.7$ it transitions from extinction to non-spreading solutions.

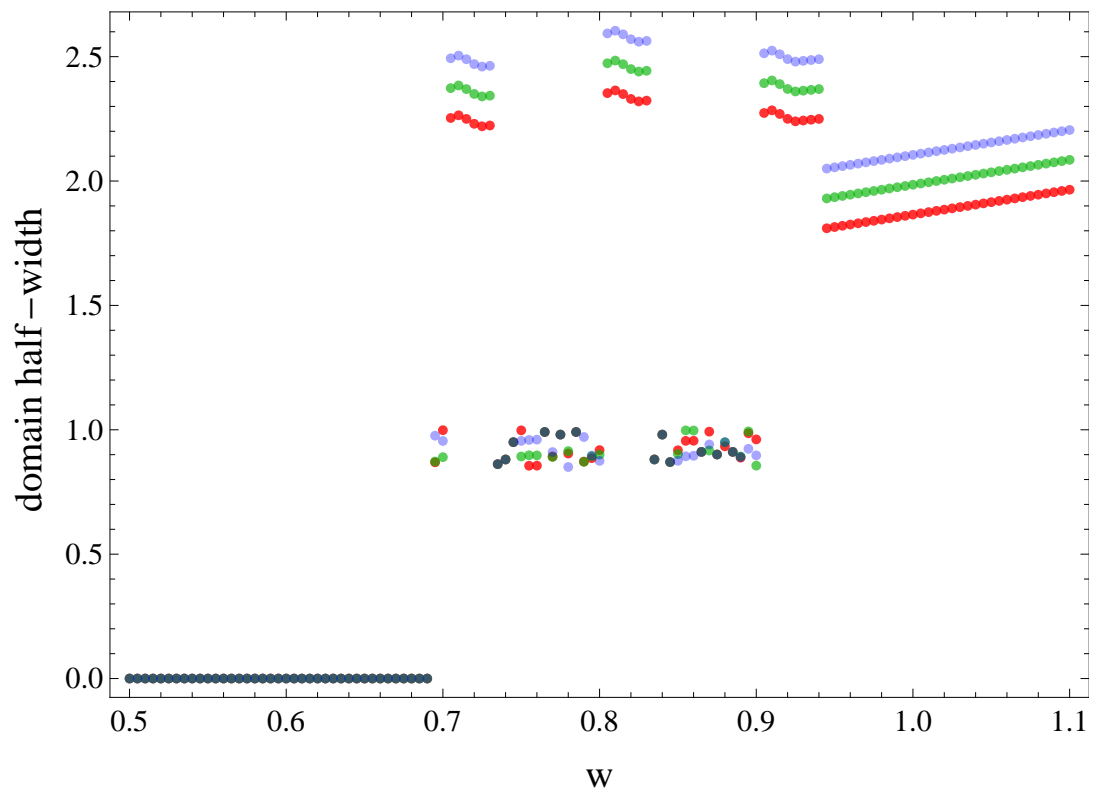

(a)

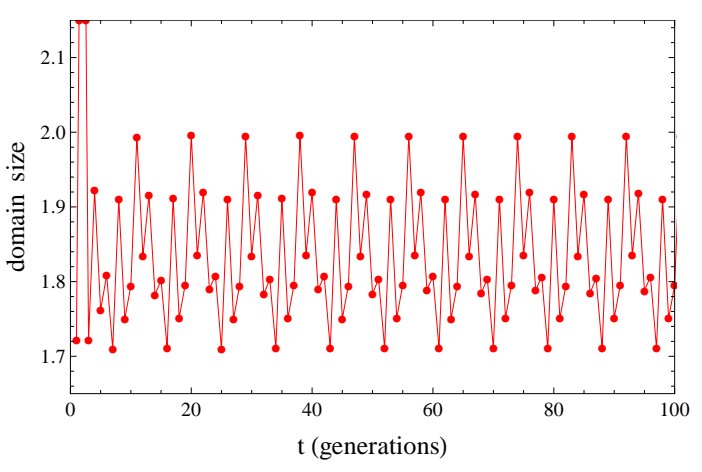

(b) domain vs. $t$ for $w=0.86$

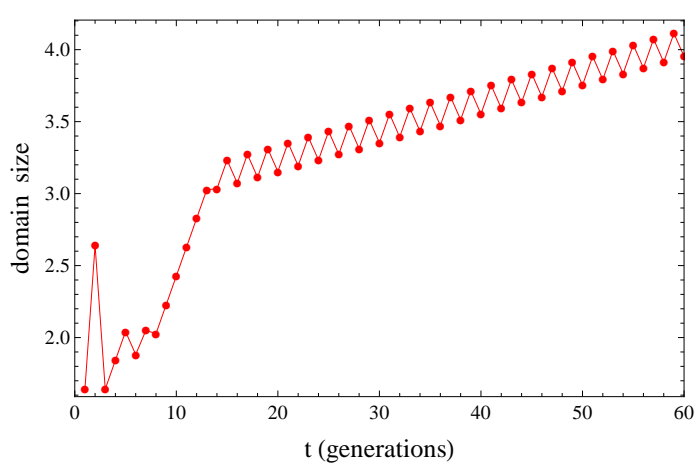

(c) domain vs. $t$ for $w=0.82$

Figure 4.10: Domain size after 88 iterations (red), 100 iterations (green), and 112 iterations (blue) as a function of initial domain half-width. In sub-figure (b) a the domain vs. time plot is shown for $w=0.86$. In sub-figure (c) the domain vs time plot is shown for $w=0.82$. Parameter values used are $\left(n_{0}, n_{1}, n_{2}\right)=(1,1.7,2.5)$.

Finally in Figure 4.11 we see an irregular pattern of non-spreading solutions and 
regions of extinction. This pattern continues even for larger values of $w$ then what are displayed on the graph.

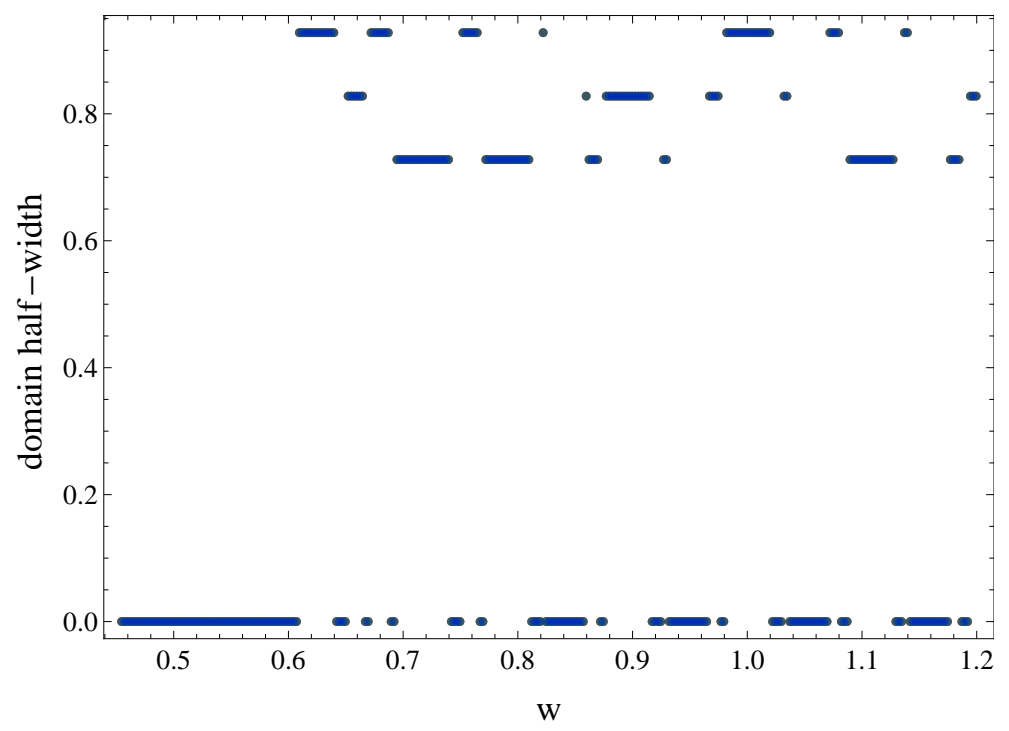

(a)

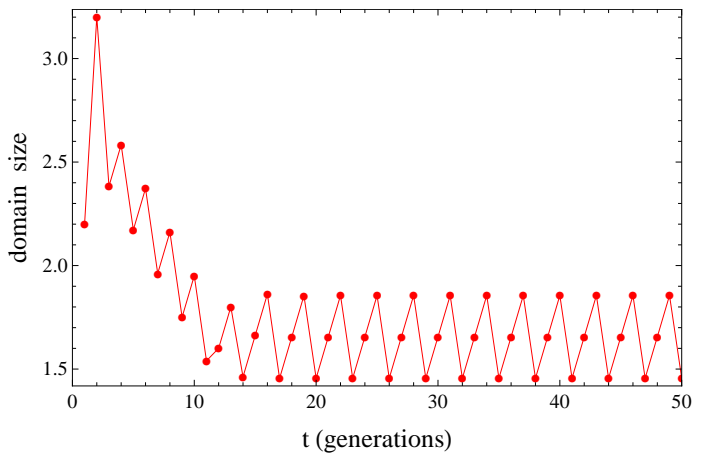

(b) domain vs. $t$ for $w=1.1$

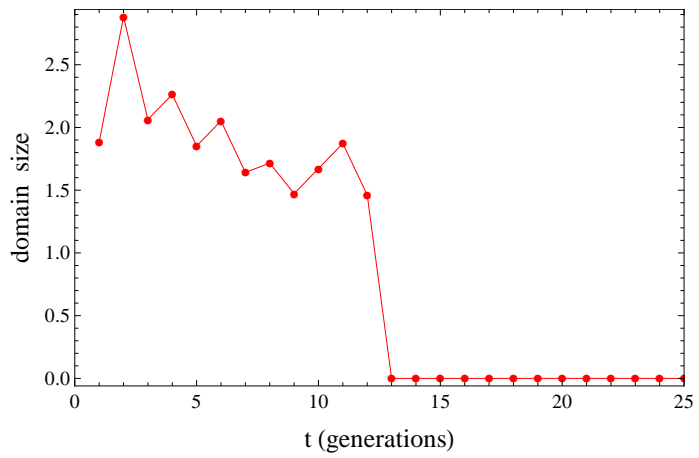

(c) domain vs. $t$ for $w=.94$

Figure 4.11: In sub-figure (a) domain size after 88 iterations (red), 100 iterations (green), and 112 iterations (blue) are depicted as a function of initial domain half-width. In subfigure (b) the domain size vs. time is depicted for a $w$ with a non-spreading solution, in sub-figure (c) the domain size vs. time is depicted for a $w$ producing extinction. Parameter values used are $\left(n_{0}, n_{1}, n_{2}\right)=(1.1,1.4,2.5)$. 


\subsubsection{Gaussian dispersal with piecewise constant fecundity function}

In Section 3.2 we analytically demonstrated the existence of equilibrium solutions in the form $k * G_{\{a, b\}}$ when $k$ is the Gaussian distribution. In this section we will examine the evolution of perturbed equilibria. As in Section 4.1, we will consider initial data in the

form $k * \widetilde{G}_{\left\{-b_{1},-a_{1}, a_{2}, b_{2}\right\}}$ where $\widetilde{G}$ is defined as in Eq. 4.1. Since the Gaussian distribution does not have compact support, we must define domain-size slightly differently then in Section 4.1. In this section we define the reference domain-size of $u_{i}(x)$ to be the length of the interval where $u_{i}(x)>1$ in the uni-modal case, The threshold of $u_{i}(x)=1$ is chosen as that corresponds to the Allee threshold. More generally we define the reference domain size to be

$$
\sup \left\{x \mid u_{i}(x)>1\right\}-\inf \left\{x \mid u_{i}(x)>1\right\}
$$

As with the uniform case, we see a wide variety of phenomena. We have found instances of perturbed solutions converging to period- 2 orbits, period-4 orbits, irregular orbits, and extinction. For parameter values with 2 distinct equilibria (blue regions in Figure 3.8) we observe that the perturbed solutions converge to the same pattern of oscillation regardless of which perturbed equilibrium the solution was initiated with. We also do not observe any distinction between the patterns of periodic oscillations that occur with the 1-equilibrium parameters versus the 2-equilibrium parameters. Stated slightly differently, for a given pattern of oscillation, instances of parameters exhibiting that type of oscillation can be found in both the blue and salmon regions of Figure 3.8. In contrast to the uniform-kernel case, we did not observe spreading solutions for small perturbations.

In Figure 4.12 a set of parameters with an attracting period-2 orbit is depicted. Both the equilibriums, depicted by the dashed-curves in sub-Figure (b), when perturbed stabilize to the same period- 2 pattern. In Figure 4.13 a case where a single equilibrium is attracted to a period-4 orbit is depicted. Finally in Figure 4.14 a set of parameters that 
develops an irregular, but non-spreading orbit is depicted. A high sensitivity to initial conditions is observed for the parameters in Figure 4.14, suggesting chaotic dynamics. 


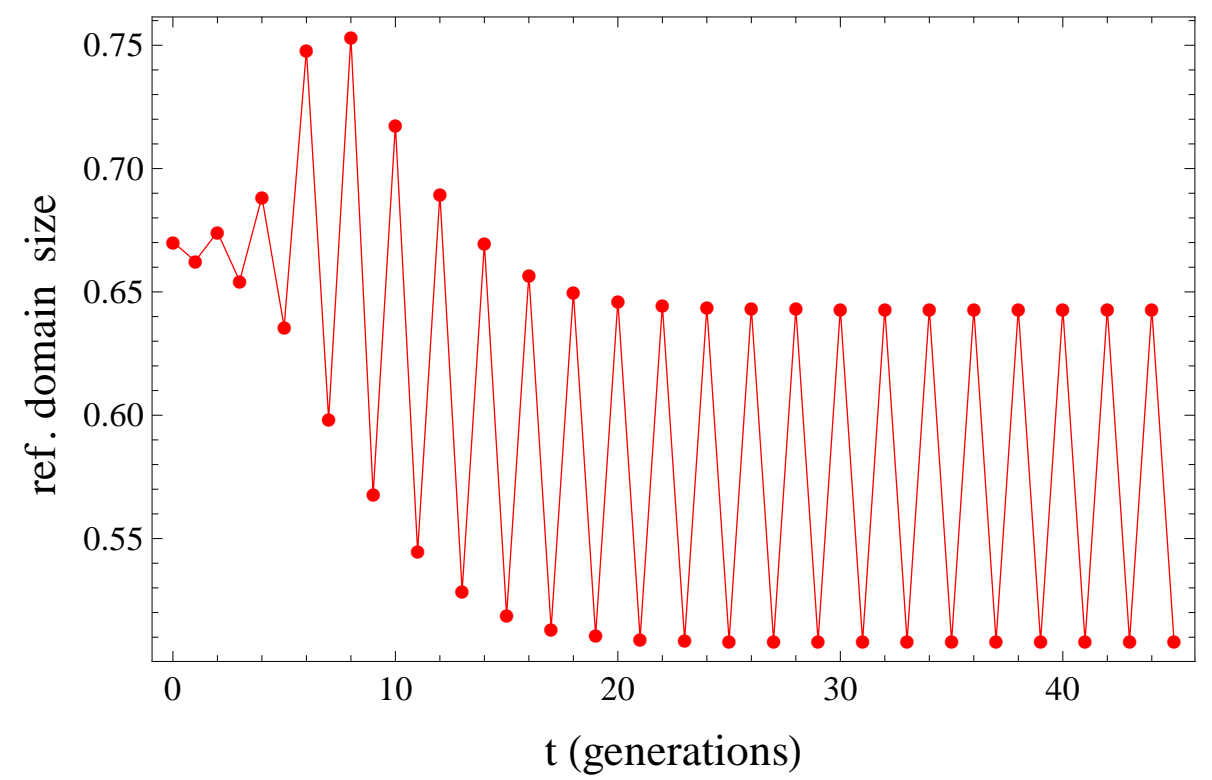

(a) ref. domain-size vs. time

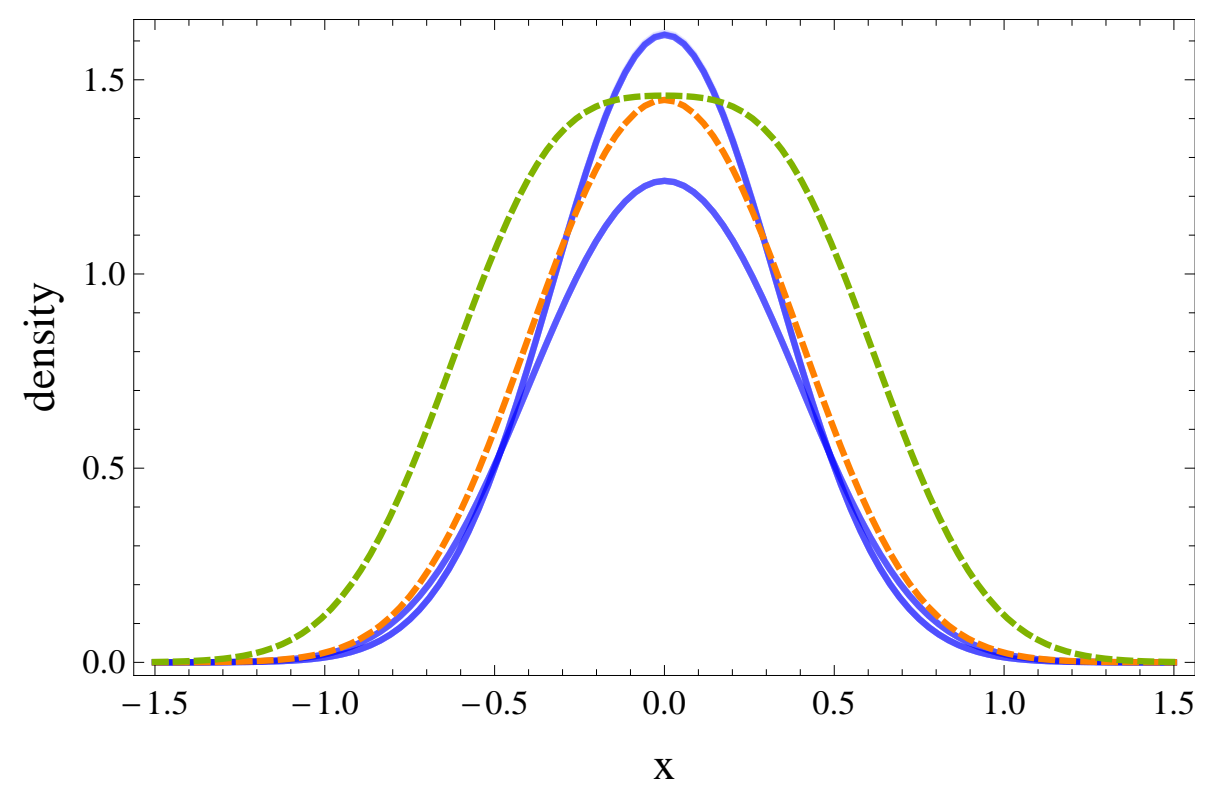

(b) Plot of $u_{20}(x)$ through $u_{40}(x)$ vs. $x$. Equilibriums are the dashed curves, period-2 curves are in blue.

Figure 4.12: A case where perturbations lead to period-2 oscillations. $\left\{n_{0}, n_{1}, n_{2}\right\}=$ $\{1.25,1.36,2.6\}$. The equilibriums are $k * G_{\{0.1399,0.3322\}}$ and $k * G_{\{0.3087,0.5279\}}$. The perturbed initial state used in this figure is $k * \widetilde{G}_{\{-0.3373,-0.1560,0.1374,0.3330\}}$. 


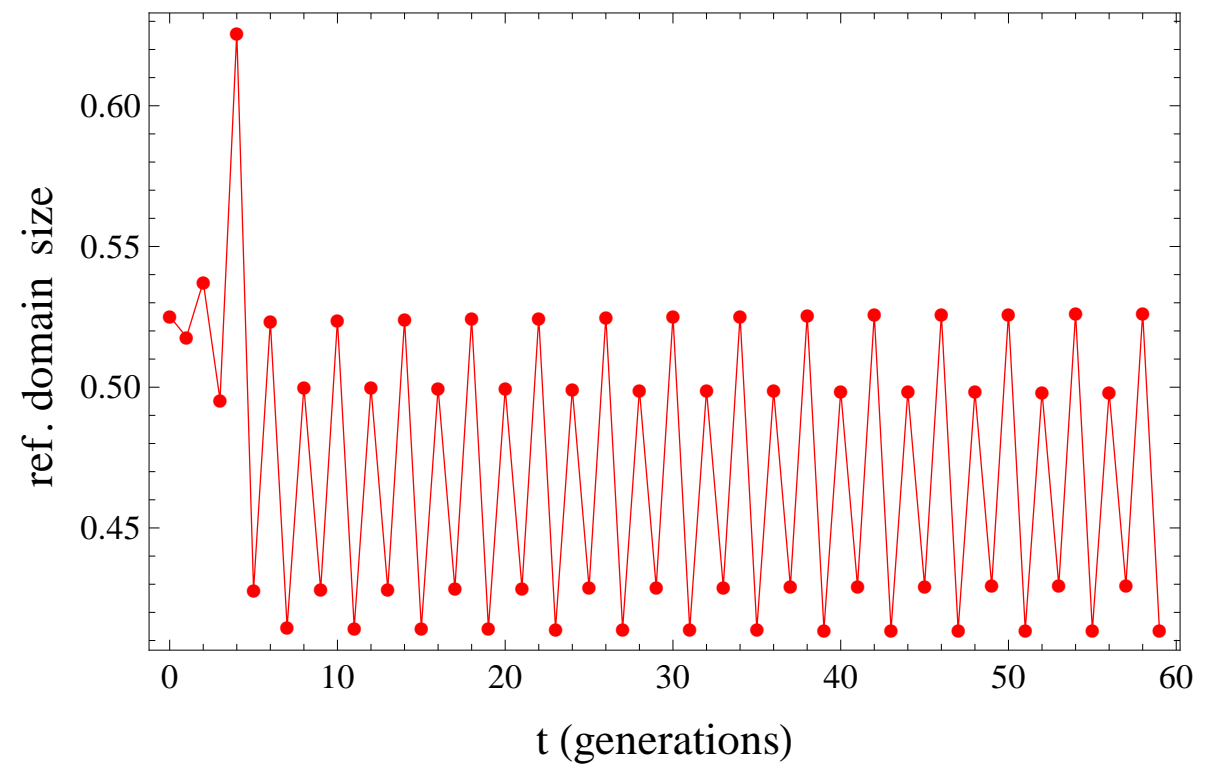

(a) ref. domain-size vs. time

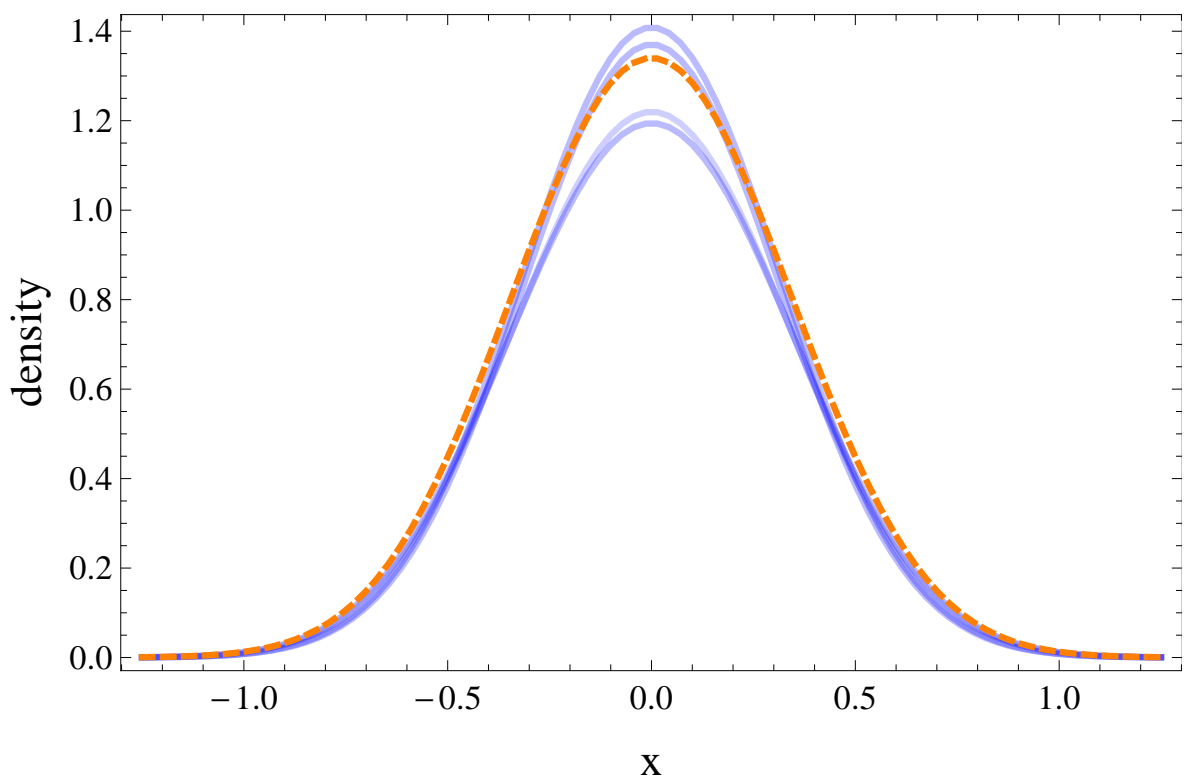

(b) Plot of $u_{10}(x)$ through $u_{20}(x)$ vs. $x$, the single equilibrium is the dashed curve.

Figure 4.13: A case where perturbations lead to period-4 oscillations. $\left\{n_{0}, n_{1}, n_{2}\right\}=$ $\{1.25,1.3,2.6\}$. The equilibrium is $k * G_{\{0.0849,0.2618\}}$. The perturbed initial state used in the figure is $k * \widetilde{G}_{\{-0.2634,-0.0933,0.0845,0.2618\}}$. 


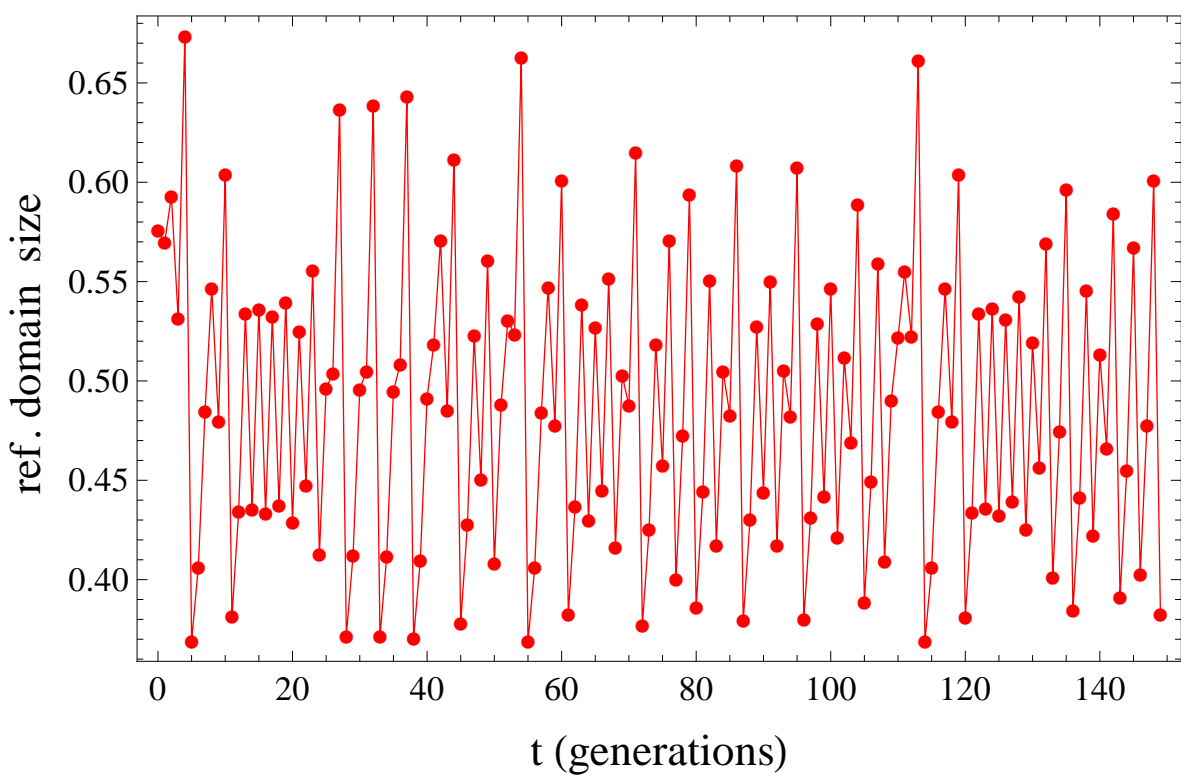

(a) ref. domain-size vs. time

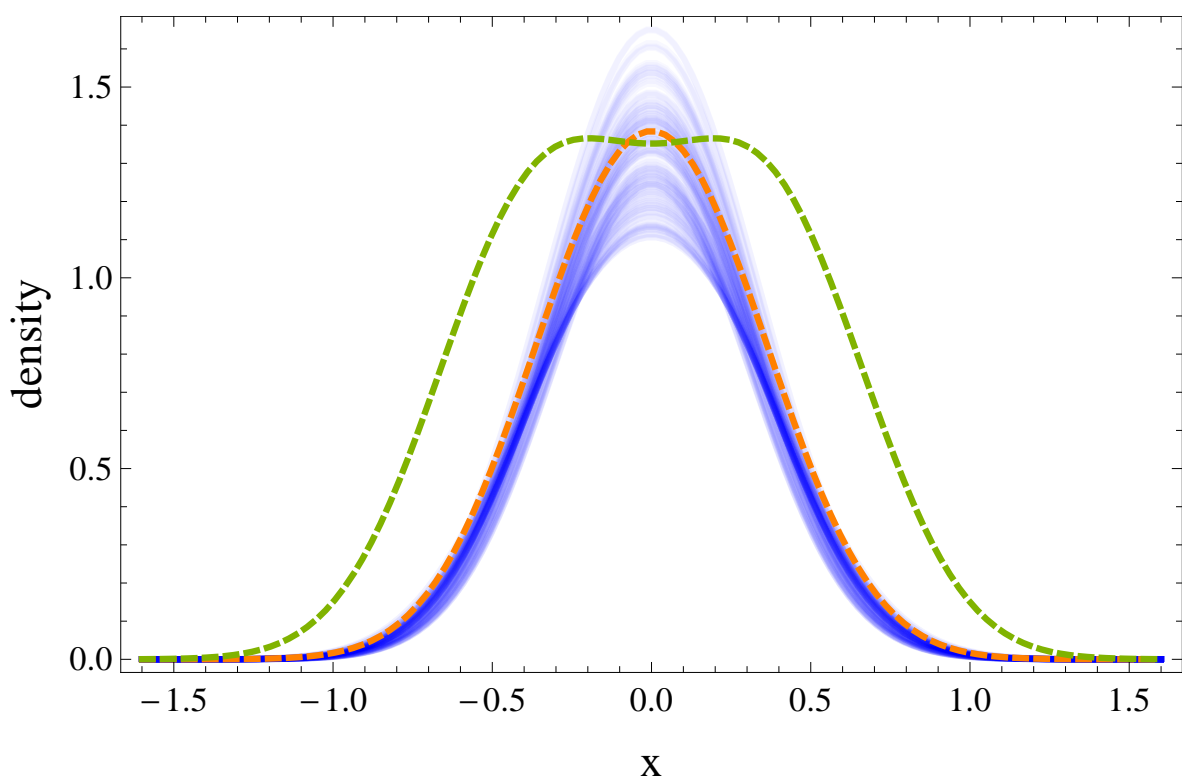

(b) Plot of $u_{20}(x)$ through $u_{150}(x)$ vs. $x$, equilibriums are the dashed curves. The $u_{i}(x)$ (in blue) are rendered semi-transparent so as to show points of accumulation.

Figure 4.14: A case where perturbations lead to irregular oscillations. $\left\{n_{0}, n_{1}, n_{2}\right\}=$ $\{1.05,1.34,2.6\}$. The equilibriums are $k * G_{\{0.0929,0.2889\}}$ and $k * G_{\{0.3091,0.5576\}}$. The perturbed initial state used in this figure is $k * \widetilde{G}_{\{-0.2866,-0.0884,0.1011,0.2891\}}$. 


\subsubsection{Leptokurtic dispersal kernel with piecewise constant fecundity function}

In Section 3.0.3 we demonstrated the existence of equilibrium solutions for leptokurtic exponential power kernels $(\gamma<2)$ with the growth function defined as in Eq. 3.1. We use the leptokurtic kernel

$$
k(x)=1.6488 \exp \left(-5.1362|x|^{1.5}\right)
$$

We then follow the methodology used in Section 4.0.4.1 and 4.0.5, with initial data in the form $k * \widetilde{G}_{\left\{-b_{1},-a_{1}, a_{2}, b_{2}\right\}}$ where $\widetilde{G}$ is defined as in Eq. 4.1.

Similar to the Uniform and Gaussian case, for some values of $\left(n_{0}, n_{1}, n_{2}\right)$ we see stable periodic orbits of varying periodicities emerge from perturbed equilibria. For instance in Figures 4.15, 4.17, and 4.18 we see attracting orbits of periodicity 2, 4, and 6 respectively. Unlike what was observed for the Uniform and Gaussian case observe some parameters appear to produce asymptotically stable equilibrium, as is shown in Figure 4.16. Of the parameter space producing equilibria for the $\gamma=1.5$ kernel (see Figure 3.12), a larger portion appear to be unstable equilibrium where perturbations lead to extinction. For instance for all equilibrium producing parameters tested where $n_{0}<1$, we found the perturbed solutions went extinct. 


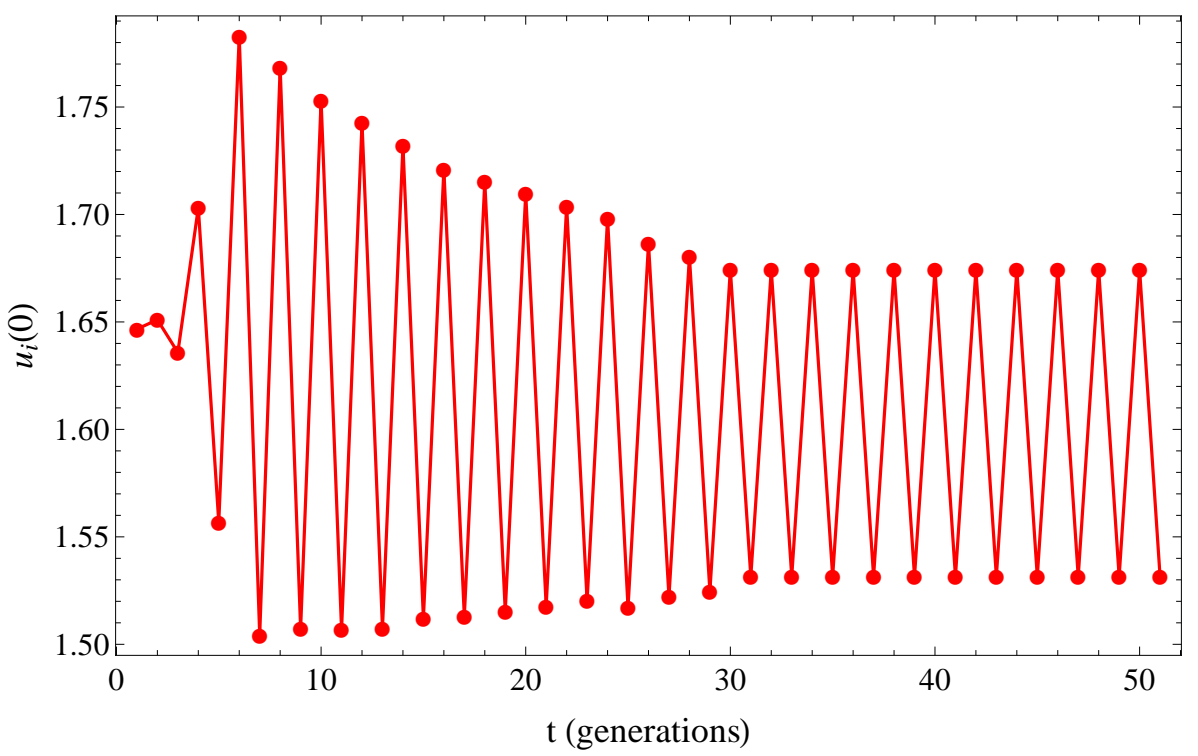

(a) $u_{t}(0)$ vs. $t$

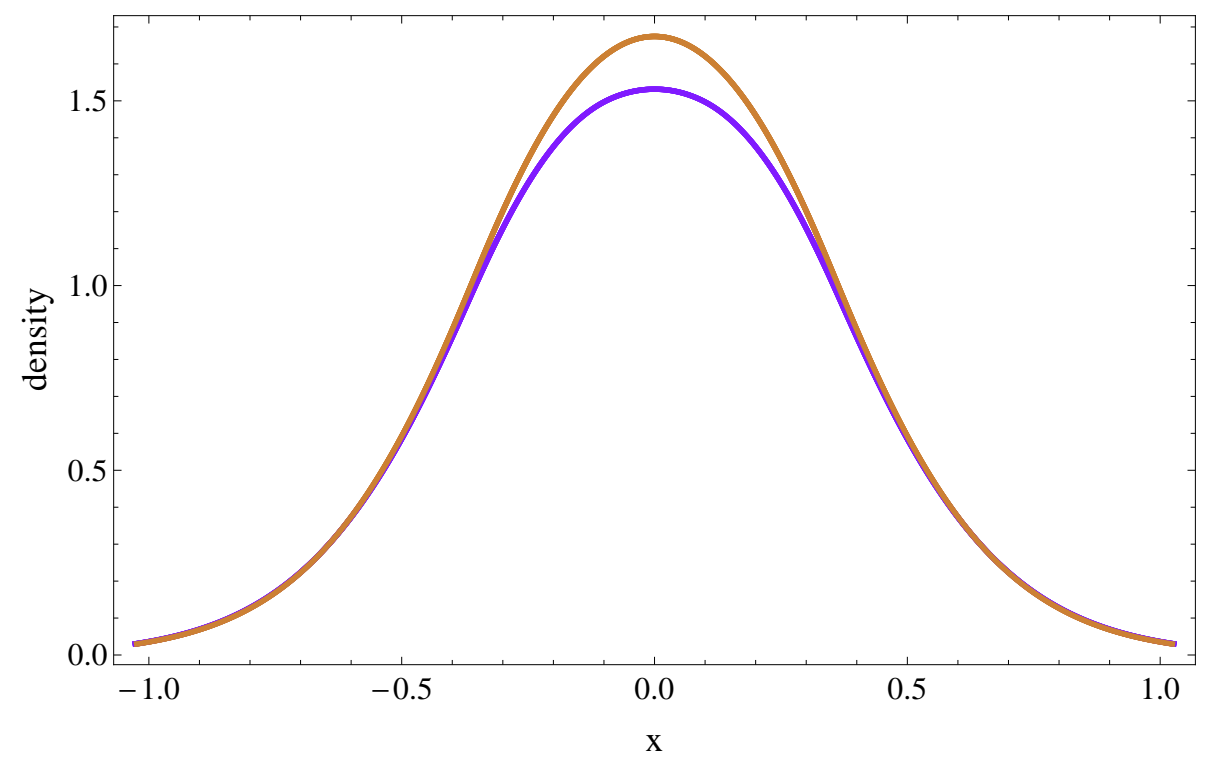

(b) $u_{40}(x)$ through $u_{50}(x)$

Figure 4.15: Perturbations leading to a period-2 oscillations. $\left\{n_{0}, n_{1}, n_{2}\right\}=$ $\{1.53,1.63,2.1\}$. The equilibria is $k * G_{\{0.0830,0.4149\}}$. The perturbed initial state used in this figure is $k * \widetilde{G}_{\{-0.4124,-0.0821,0.0786,0.4122\}}$. 


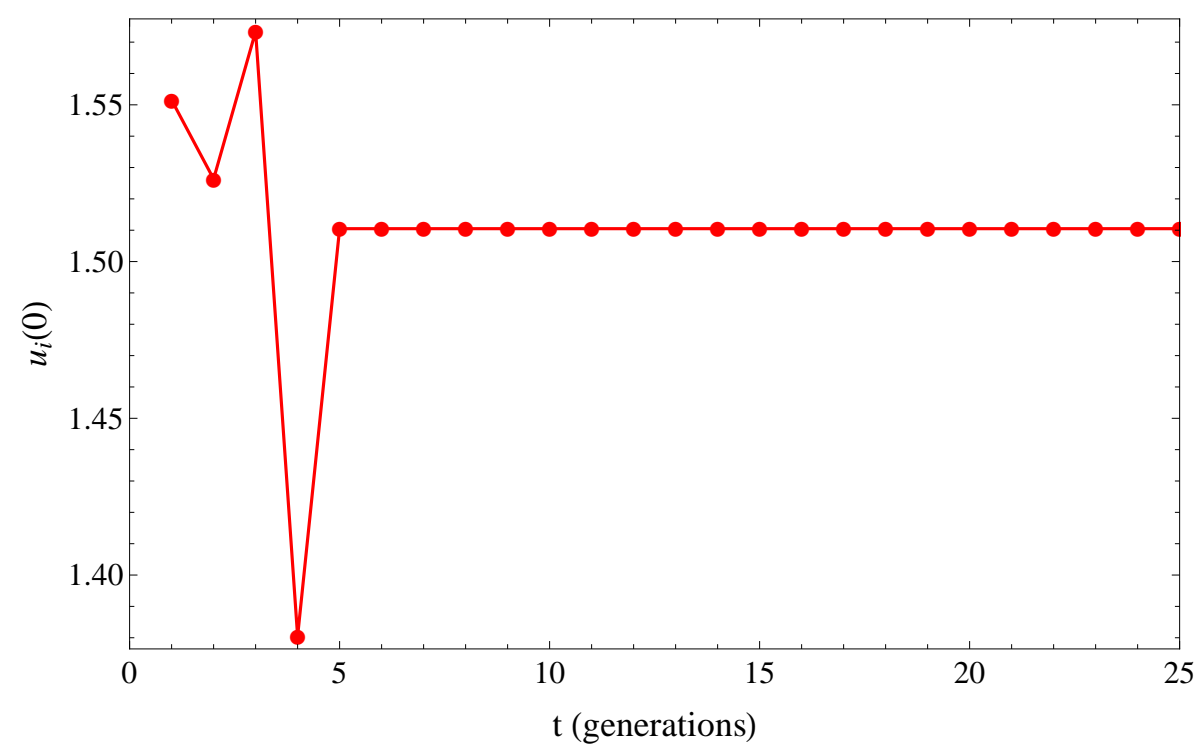

(a) $u_{t}(0)$ vs. $t$

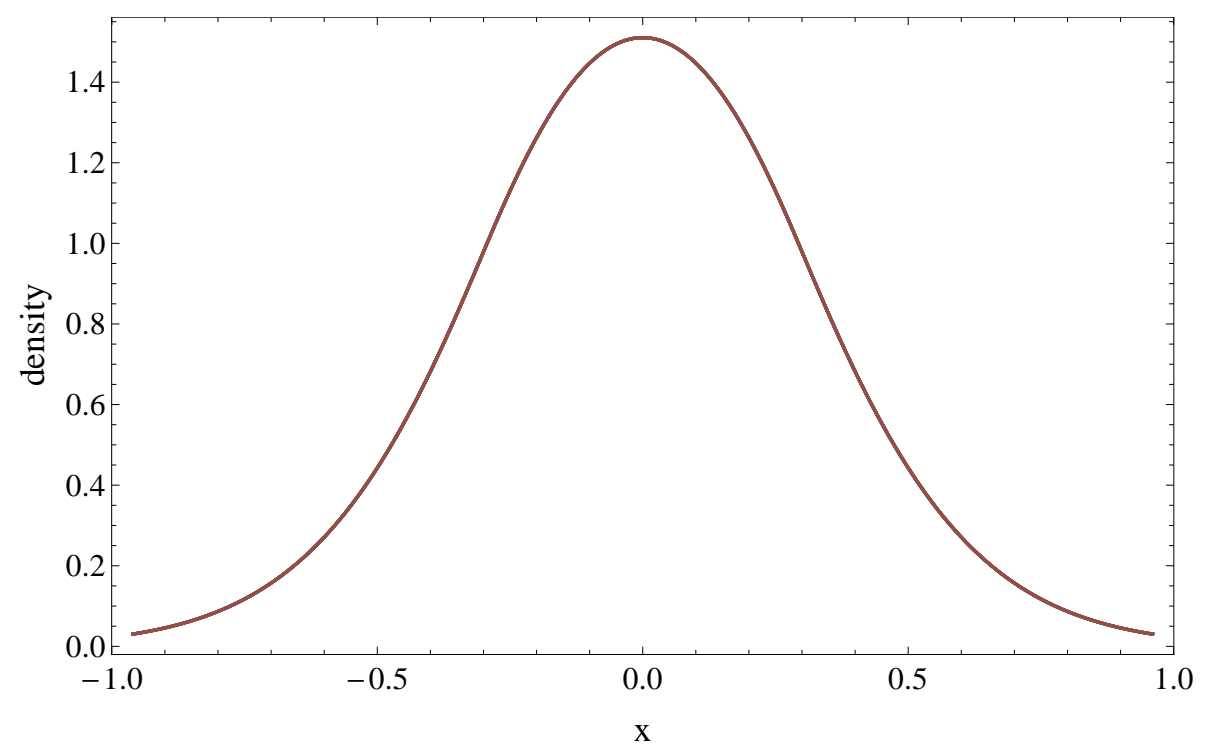

(b) $u_{20}(x)$ through $u_{25}(x)$

Figure 4.16: Perturbations lead to a stable equilibrium. $\left\{n_{0}, n_{1}, n_{2}\right\}=\{1.1,1.55,2.1\}$. The equilibria is $k * G_{\{0.0092,0.3181\}}$. The perturbed initial state used in this figure is $k * \widetilde{G}_{\{-0.3181,-0.0097,0.0069,0.3180\}}$. 


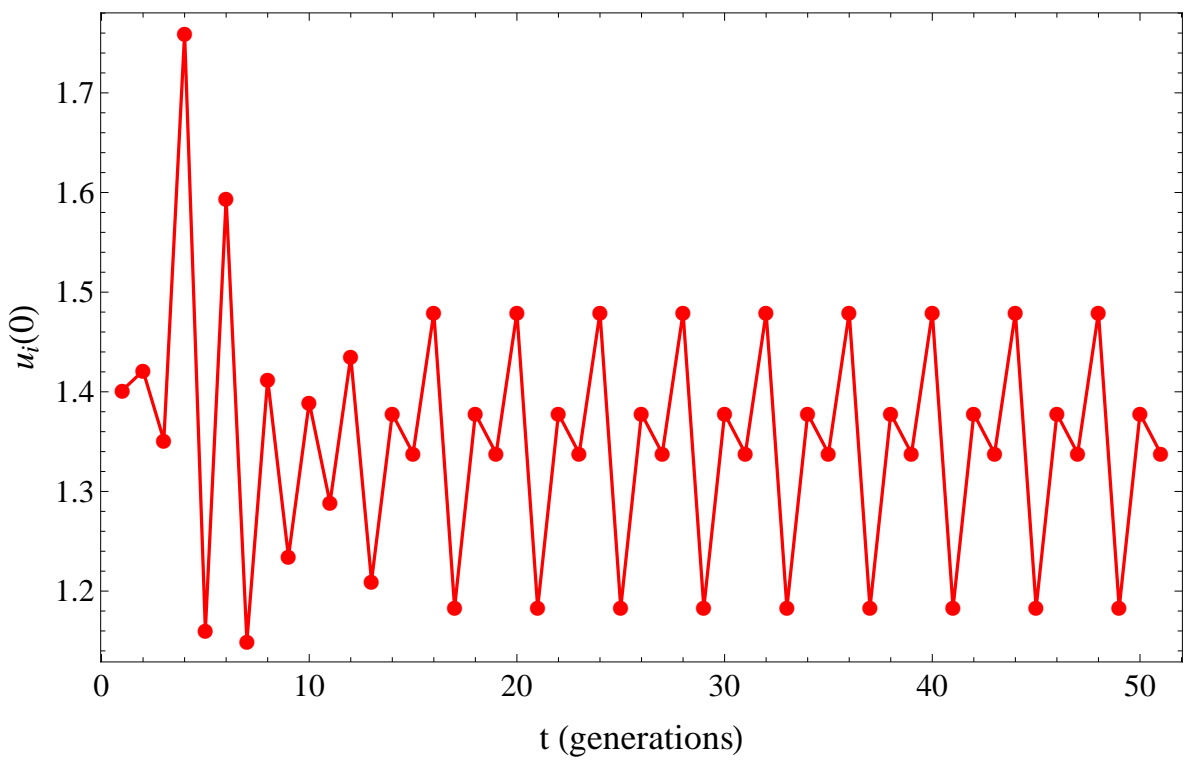

(a) $u_{t}(0)$ vs. $t$

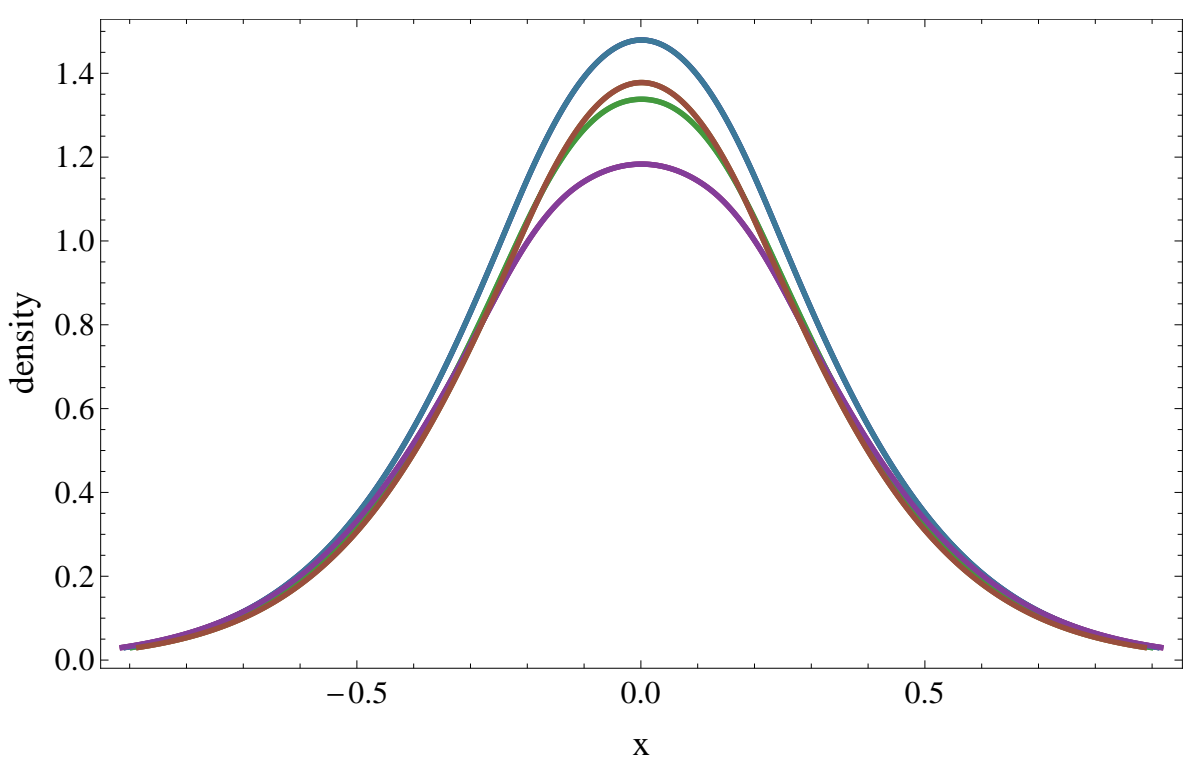

(b) $u_{40}(x)$ through $u_{50}(x)$

Figure 4.17: Perturbations leading to period-4 oscillations. $\left\{n_{0}, n_{1}, n_{2}\right\}=$ $\{1.24,1.37,2.5\}$. The equilibria is $k * G_{\{0.0977,0.2904\}}$. The perturbed initial state used in this figure is $k * \widetilde{G}_{\{-0.2943,-0.1041,0.0985,0.2933\}}$. 


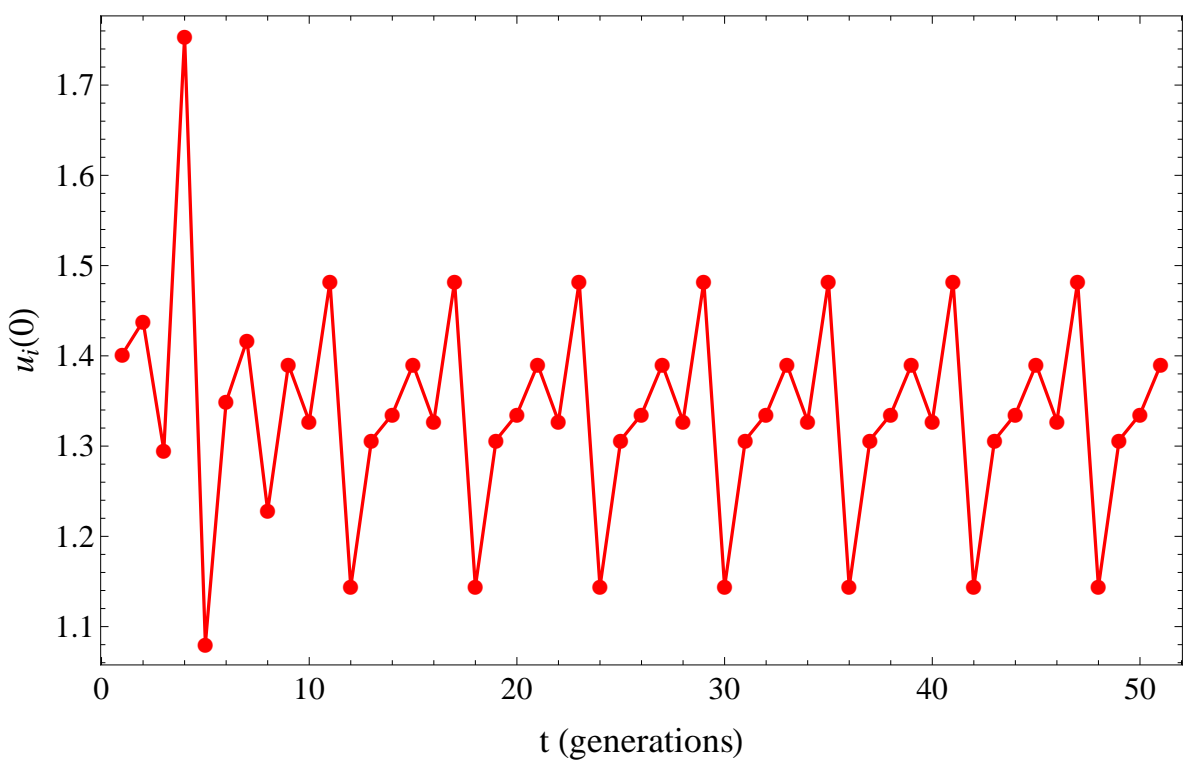

(a) $u_{t}(0)$ vs. $t$

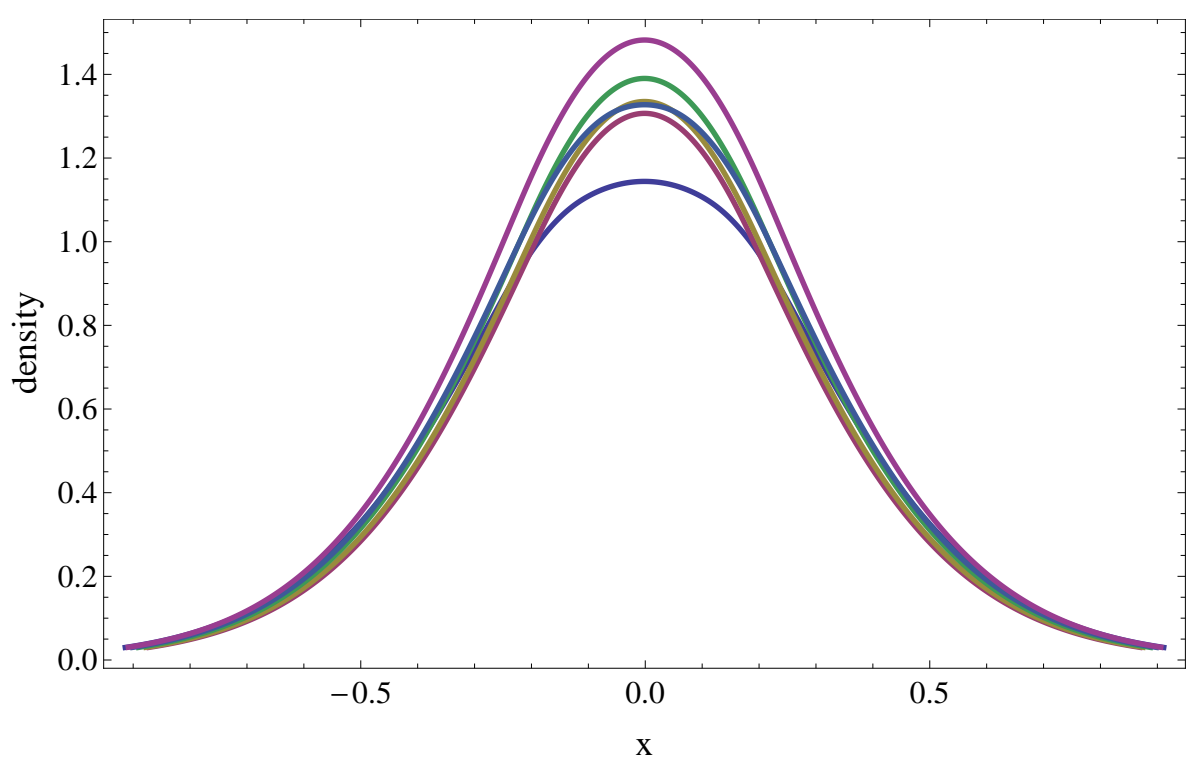

(b) $u_{40}(x)$ through $u_{50}(x)$

Figure 4.18: Perturbations leading to period-6 oscillations. $\left\{n_{0}, n_{1}, n_{2}\right\}=$ $\{1.1,1.38,2.5\}$. The equilibriums are $k * G_{\{0.0973,0.3028\}}$. The perturbed initial state used in this figure is $k * \widetilde{G}_{\{-0.2991,-0.0982,0.0938,0.2983\}}$. 


\subsubsection{Numerical results for other growth functions}

Thus far our results have focused on non-spreading solutions involving the piecewise constant growth function described in Eq. 3.1. To demonstrate that non-spreading solutions can occur for more general growth functions, we conduct numerical simulations for several variants of the Ricker growth function. Using the example of Schreiber (2003) we consider a growth function of the form $g(u)=u \exp (r(1-u)) I(u)$, where the positive density dependance at low densities (i.e. Allee effect) is encapsulated in $I(u)$. While Schreiber specifically examines a case where $I(u)$ is a Hill function with exponent 1 , we will consider the more general case where $I(u)$ is any Hill function. Throughout this section we use the exponential power distribution $k(x)=1.1109 \exp \left(-\left(\frac{x}{0.4965}\right)^{4}\right)$, whose variance is $\frac{1}{12}$ in keeping with the previous cases, and whose kurtosis is intermediate to that of the Gaussian and Uniform distributions of the same variance. Throughout, we also use Heaviside Pi (boxcar function) initial data of half-width 0.5 and height 1.25.

4.0.7.1 Truncated Ricker function We first consider the limiting case as the Hill exponent tends to infinity, and $I(u)$ becomes a step function. This truncated Ricker function was studied by Sullivan (2017) in terms of fluctuating invasion speeds for an integral-difference model. Parameterizing so that the Allee threshold occurs at $u=1$ and the second equilibrium occurs at $u=q$ we have

$$
g(u)= \begin{cases}0, & u<1 \\ u \exp (r(q-u)), & u>1\end{cases}
$$

In Figure 4.19 we show an example of an asymptotically stable non-spreading solution with $r=1.2$ and $q=1.7$. We find that for $q=1.7$ we obtain asymptotically stable non-spreading solutions for $1.05<r<1.29$ (to 2 decimal places). For $r>1.05$ extinction occurs and for $r<1.29$ chaotic spreading solutions occur. Similarly, we find for $r=1.2$ we obtain asymptotically stable non-spreading solutions for roughly $1.65<$ $q<1.72$ (to 2 decimal places). For $q<1.65$ extinction occurs and for $r>1.72$ chaotic 
spreading solutions occur. 


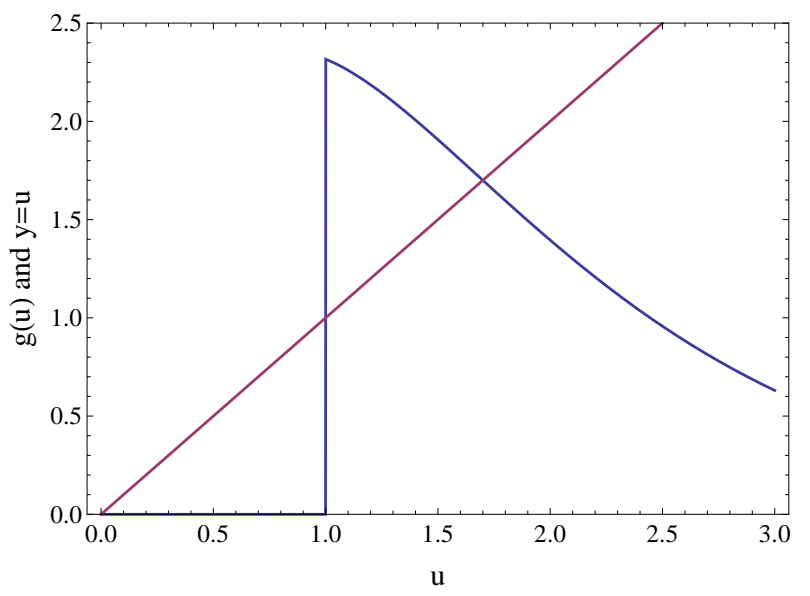

(a) $g(u)$

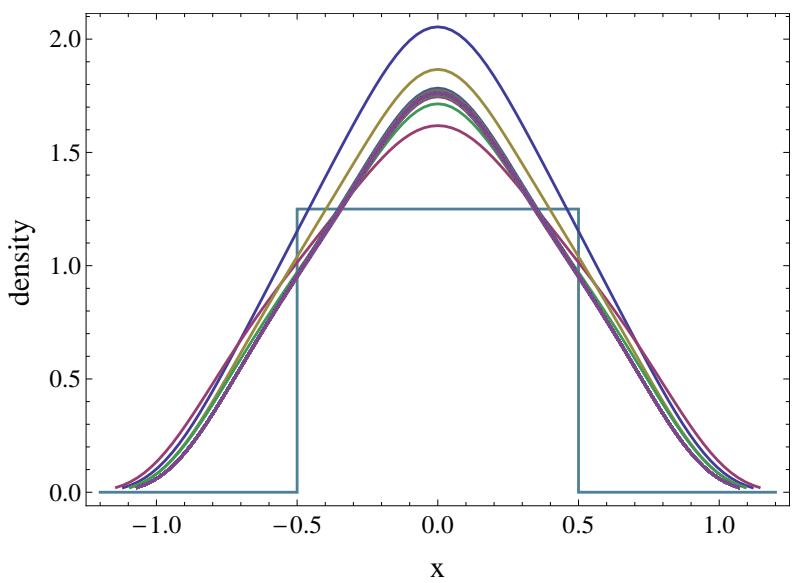

(b) $u_{0}(x)$ through $u_{100}(x)$

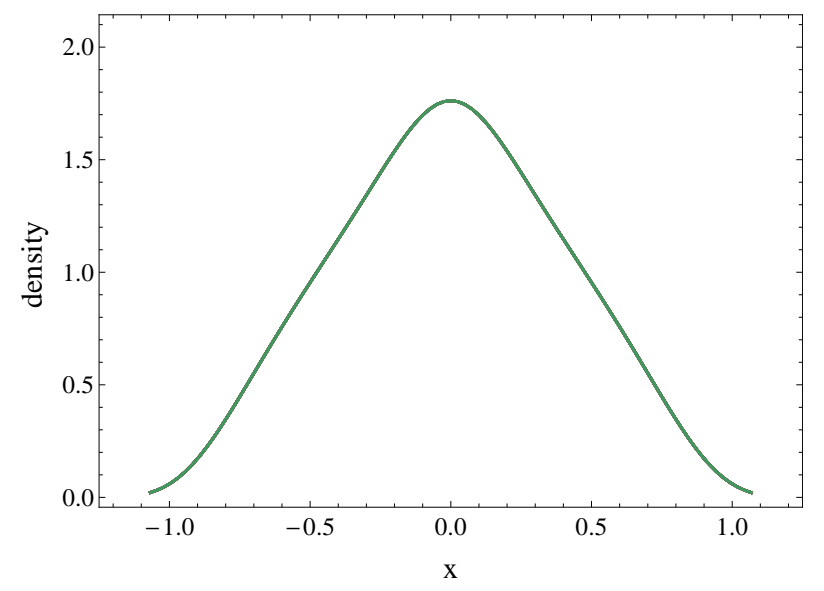

(c) $u_{80}(x)$ through $u_{100}(x)$

Figure 4.19: Plots of the growth function, transient iterations, and asymptotic attractor for the truncated Ricker function with $r=1.2$ and $q=1.7$. 
4.0.7.2 Hill-Ricker function We next consider

$$
g(u)=u \exp (r(1-u))\left(\frac{\left(1+1.3^{7}\right)\left(\frac{u}{1.3}\right)^{7}}{1+\left(\frac{u}{1.3}\right)^{7}}\right)
$$

where we leave $r$ as a bifurcation parameter. An example of this function with $r=2.5$ is depicted in Figure 4.20.

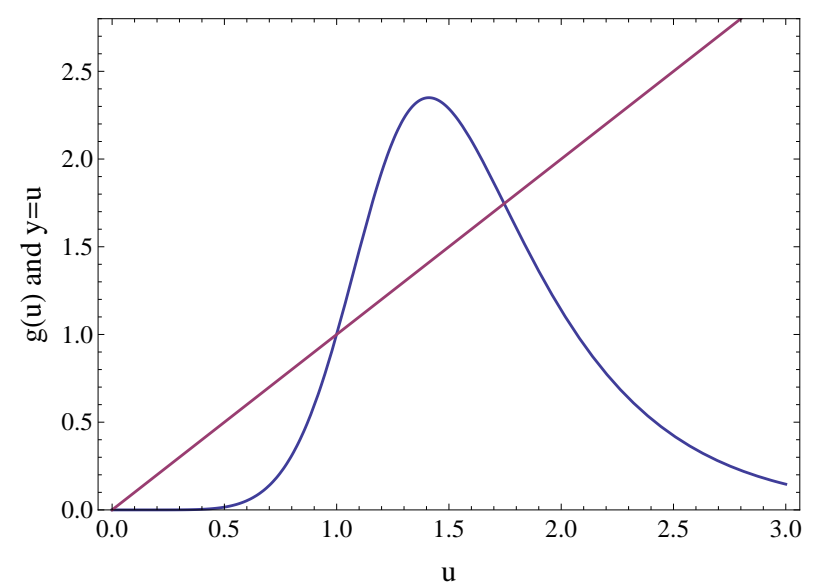

Figure 4.20: The Hill-Ricker growth function $g(u)=u \exp (2.5(1-$ u)) $\left(\frac{\left(1+1.3^{7}\right)\left(\frac{u}{1.3}\right)^{7}}{1+\left(\frac{u}{1.3}\right)^{7}}\right)$.

For $r<2.44$ (to 2 decimals) we find chaotic spreading solutions.

For $2.44<r<2.64$ we find stable period 2 oscillations as depicted in Figure 4.21. 


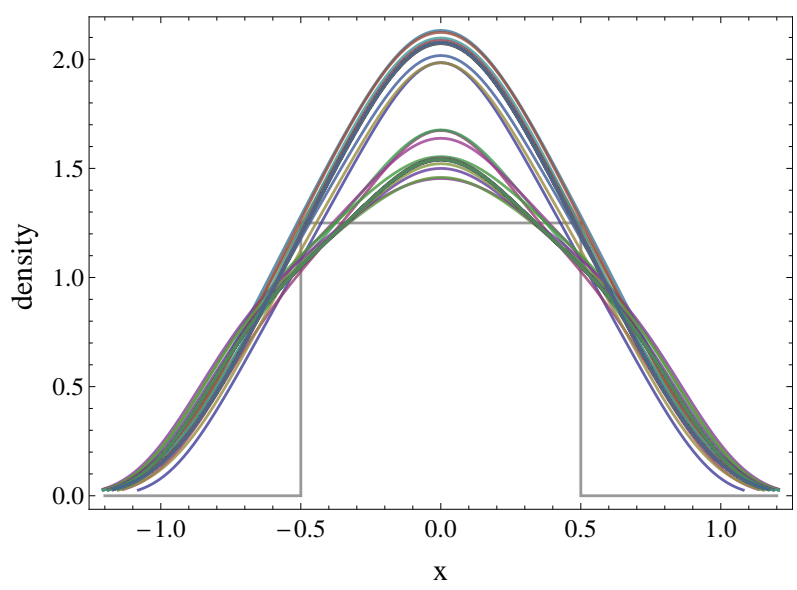

(a) $u_{0}(x)$ through $u_{100}(x)$.

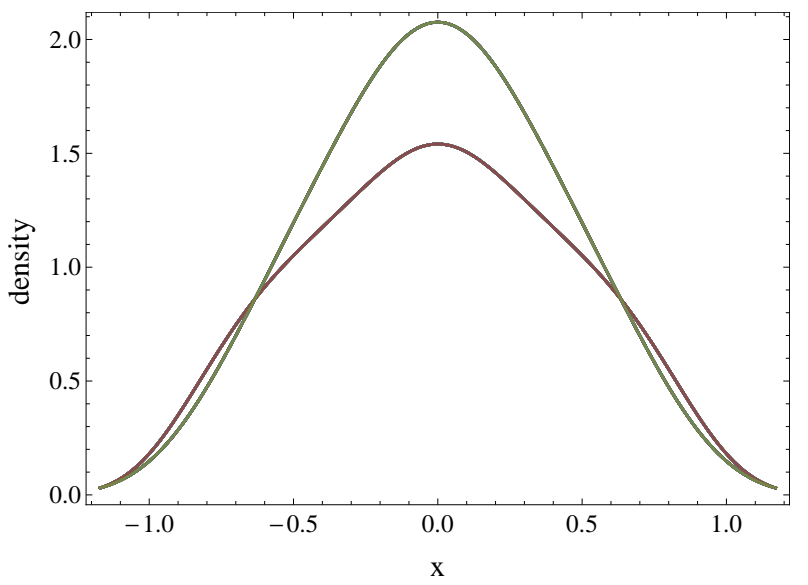

(b) $u_{80}(x)$ through $u_{100}(x)$

Figure 4.21: Plots of solution curves for $r=2.5$.

For $2.64<r<2.91$ we find a asymptotically stable equilibriums as depicted in Figure 4.22. 


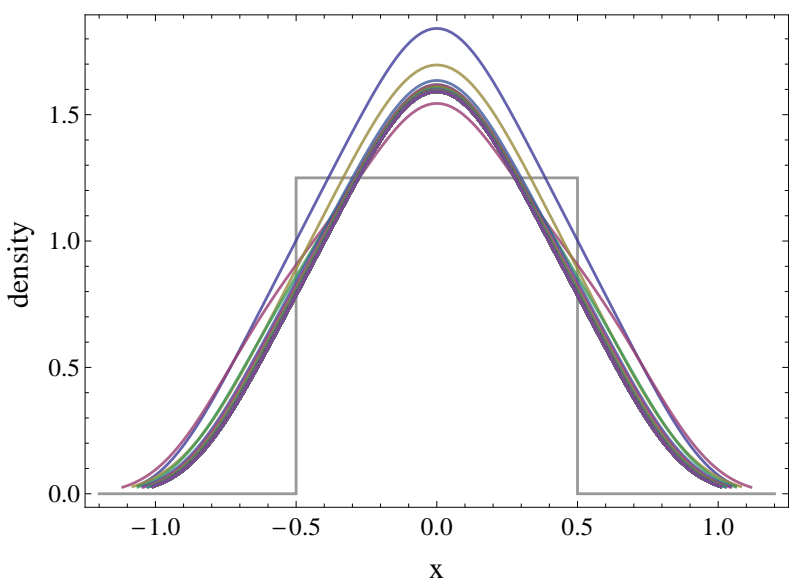

(a) $u_{0}(x)$ through $u_{100}(x)$.

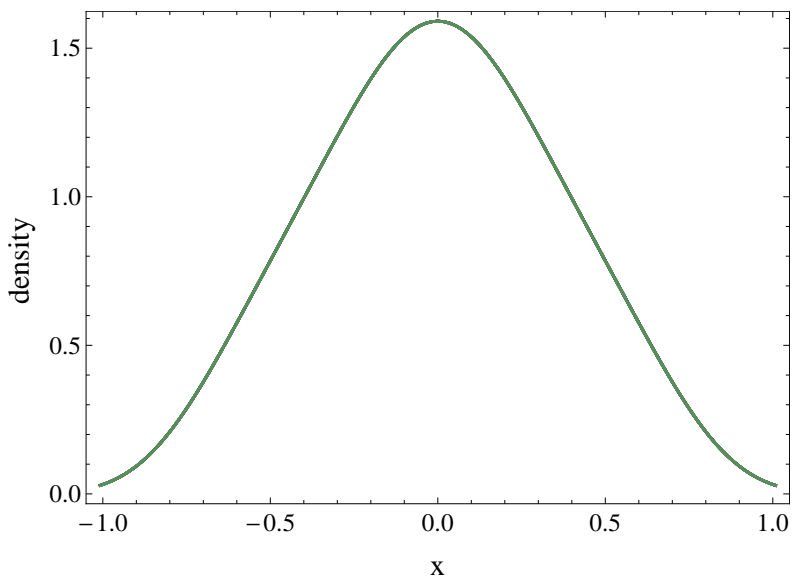

(b) $u_{80}(x)$ through $u_{100}(x)$

Figure 4.22: Plots of solution curves for $r=2.8$.

For $r>2.91$ extinction occurs.

\subsubsection{Truncated-Ricker function with linear strong-Allee effect Thus far} we have only considered growth functions with rather severe forms of strong Allee effect, such as $g(u)$ being zero on $[0,1)$, or in the case of the Hill-Ricker growth function we considered, $g(u) \sim u^{8}$ for small $u$. To demonstrate that non-spreading solutions can occur even for linear strong Allee effect, we consider the following growth function: 


$$
g(u)= \begin{cases}\rho u, & u<1 \\ u \exp (1.35(1.5-u)), & u>1 .\end{cases}
$$

We find that for $0 \leq \rho \leq 0.55$ there are asymptotically stable non-spreading solutions. The solutions for $\rho=0.4$ are depicted in Figure 4.23. For $\rho>.55$ non-chaotic spreading solutions occur. 


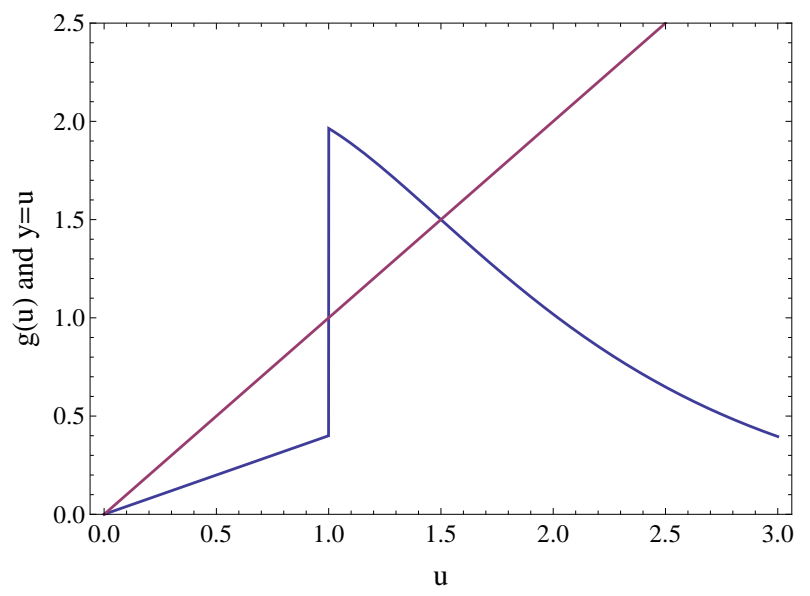

(a) $g(u)$

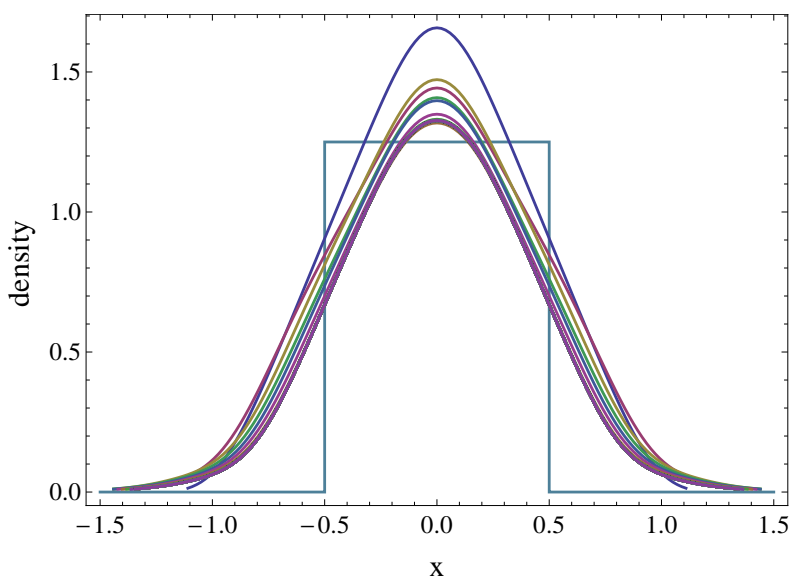

(b) $u_{0}(x)$ through $u_{100}(x)$

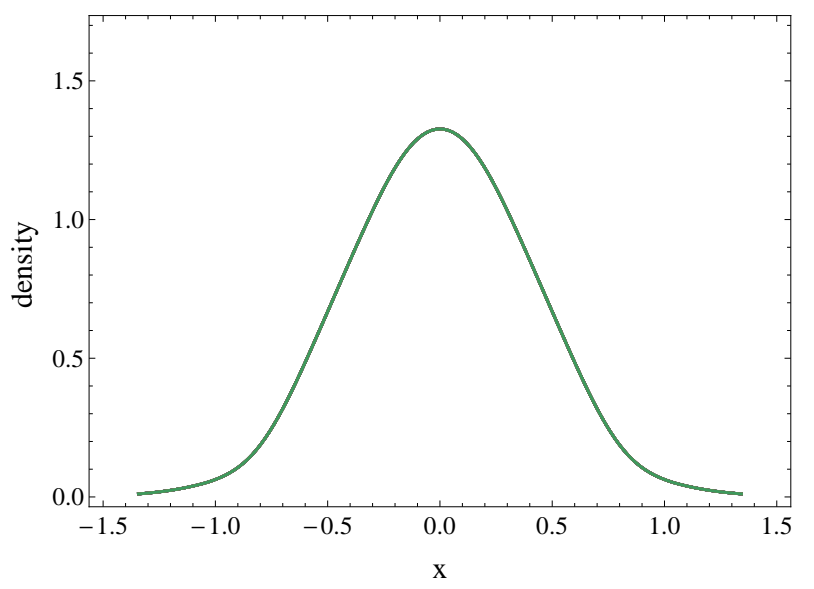

(c) $u_{80}(x)$ through $u_{100}(x)$

Figure 4.23: Plots of the growth function, transient iterations, and asymptotic attractor for the growth function in Eq. 4.3 with $\rho=0.4$. 


\subsubsection{Patch Formation}

In section 4.0.7 we have considered the evolution of the population with relatively simple initial data, namely a boxcar function whose support, $(-0.5,0.5)$, is comparable in length scale to that of a typical non-spreading solution, such as that seen in Figure 4.22. In this section we wish to examine pattern emergence from more complex initial data, in the case of growth functions which produce non-spreading solutions. We first examine the case of the evolution of two interacting non-spreading solutions. We next examine pattern emergence from perturbed constant initial data over lengths much larger then the length scale of the non-spreading solutions.

Throughout this section we will use Hill-Ricker Growth function (Eq. 4.2) and $k(x)=1.1109 \exp \left(-\left(\frac{x}{0.4965}\right)^{4}\right)$. We will examine the dynamics for both the case that $r=2.8$ and there exists exhibits a single asymptotically stable non-spreading solution (Figure 4.22), and for $r=2.5$ where there exists a stable period-2 orbit (Figure 4.21).

It should be noted that the final end-states produced by these simulations are extremely sensitive to mesh-size, however the qualitative behavior remain largely insensitive. Throughout this section the mesh-size used was $\delta=0.00664$.

4.0.8.1 Two interacting stable equilibrium To study the effects of two interacting patches in the case $r=2.8$ with a period- 1 stable attracting non-spreading solution, we let $u_{e}(x)$ be the stable attractor depicted in Figure 4.22. We determine $u_{e}(x)$ numerically by iterating until a max difference fixed point stopping criteria is met. We then initialize the simulation with

$$
u_{0}(x)=u_{e}\left(x+\frac{w}{2}\right)+u_{e}\left(x-\frac{w}{2}\right)
$$

$w$, the inter-patch spacing is then treated as a bifurcation parameter vs. the total final population. We iterate the system until the fixed point stopping criteria is reached, calling the final state $u_{\Omega}$.

Since the total population of $u_{e}$ is $\int_{-\infty}^{\infty} u_{e}(s) \mathrm{d} s=1.606$ we might expect that total 
final population, $\int_{-\infty}^{\infty} u_{\Omega}(s) \mathrm{d} s$ would be very close to an integer multiple of 1.606 depending on how many patches form. Indeed, in Figure 4.24 we see this is indeed the case. The grey dashed lines represent multiples of 1.606. We see either extinction (blue), merger into one patch (green), or two separate patches persisting (red) can occur. The fact that extinction occurs for small values of $w<0.77$ is not surprising as the near superposition of the maxima creates a very high density that is subsequently mapped below the Allee threshold by the strong overcompensation. Similarly we see for very large values of $w>1.85$ we see 2-patches persist, which is not surprising as the interaction between them is extremely weak. For intermediate values of $w$ we see there is merger into a single patch interspersed with intervals of extinction. In Figure 4.25 we show the evolution of the patches merging into a single patch. In Figure 4.26 we show the evolution of the patches collapsing into extinction.

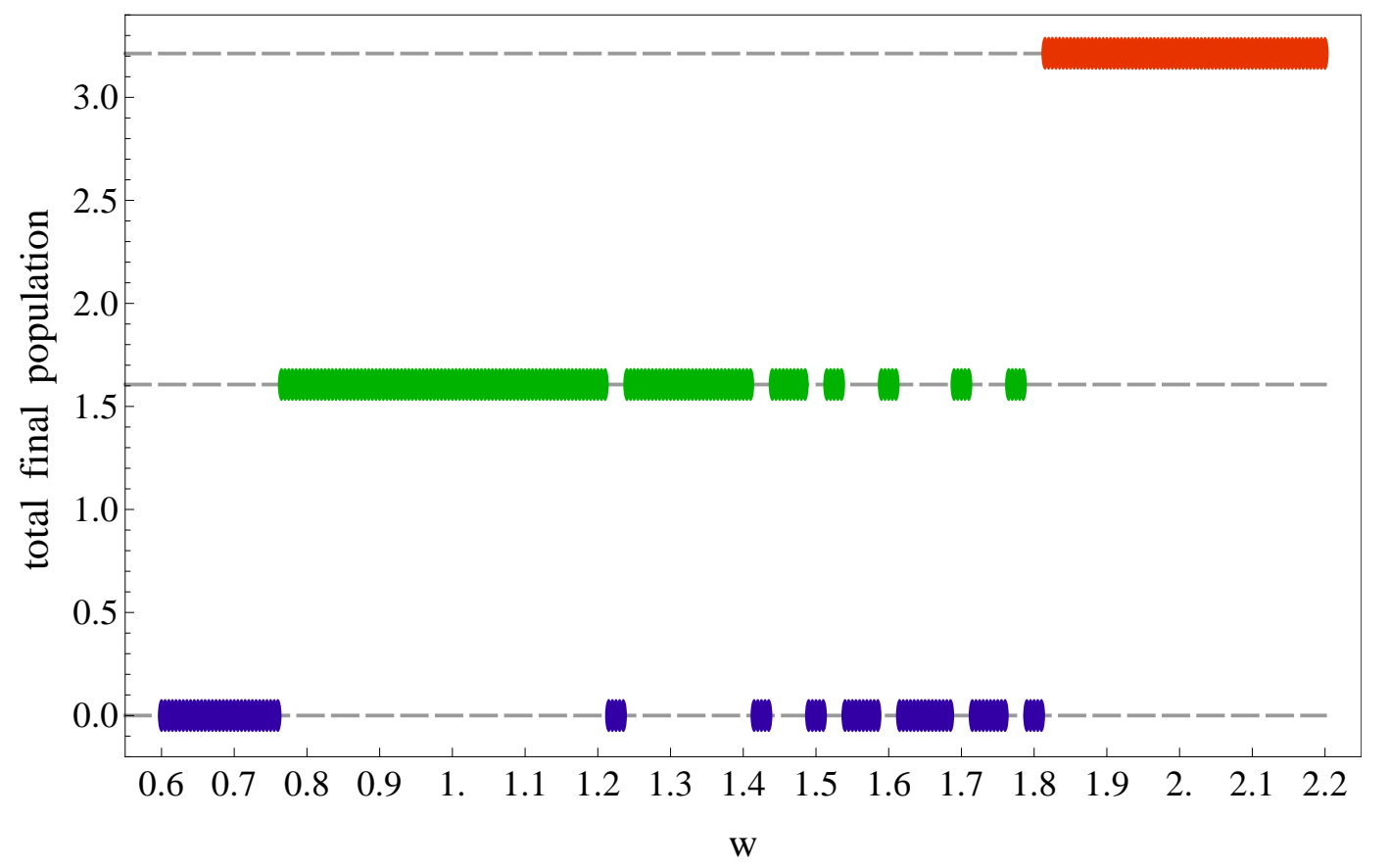

Figure 4.24: Total population of end-state versus spacing parameter $(w)$. 


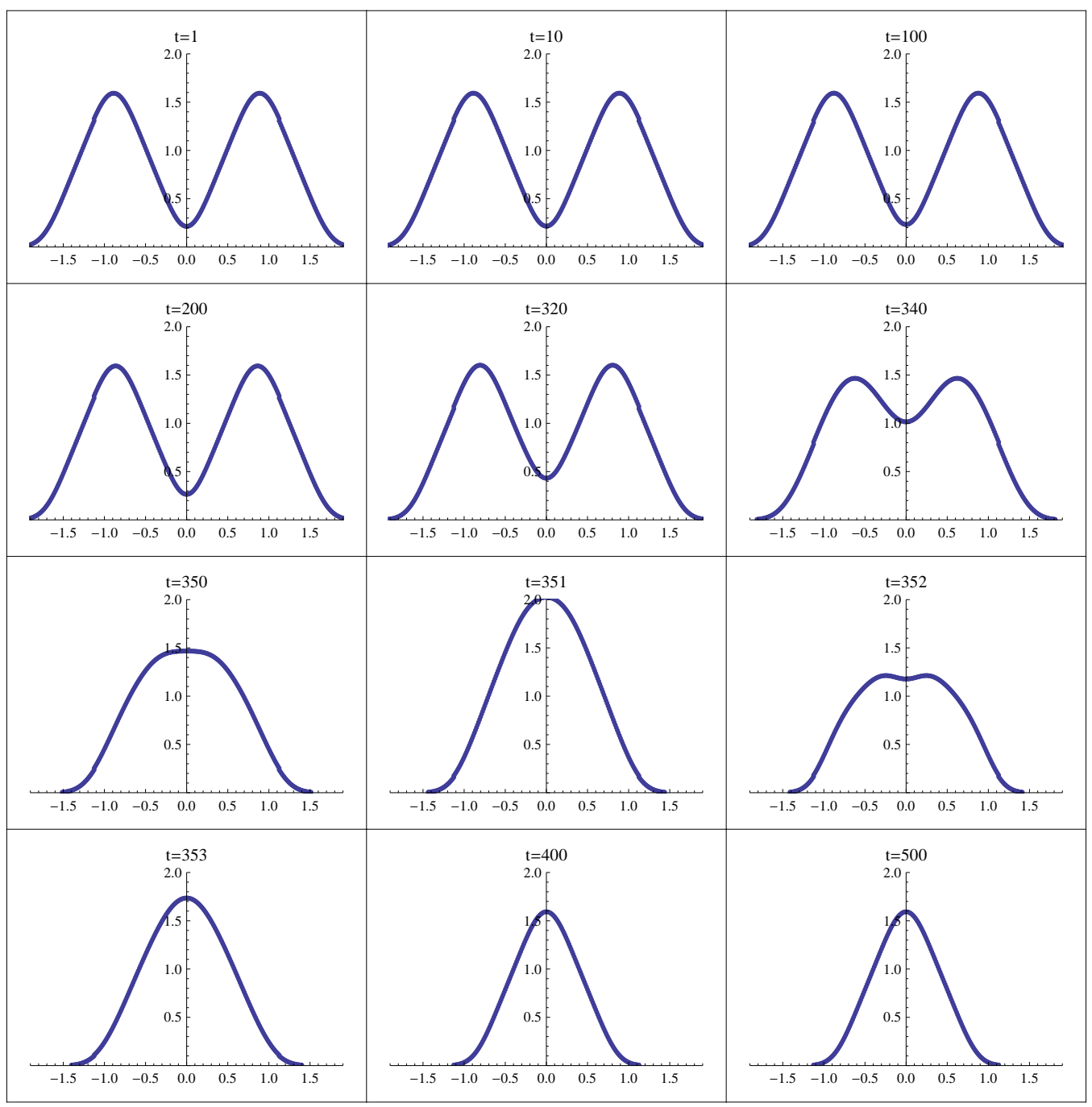

Figure 4.25: Evolution of solution ending a merger of patches. $w=1.77$ 


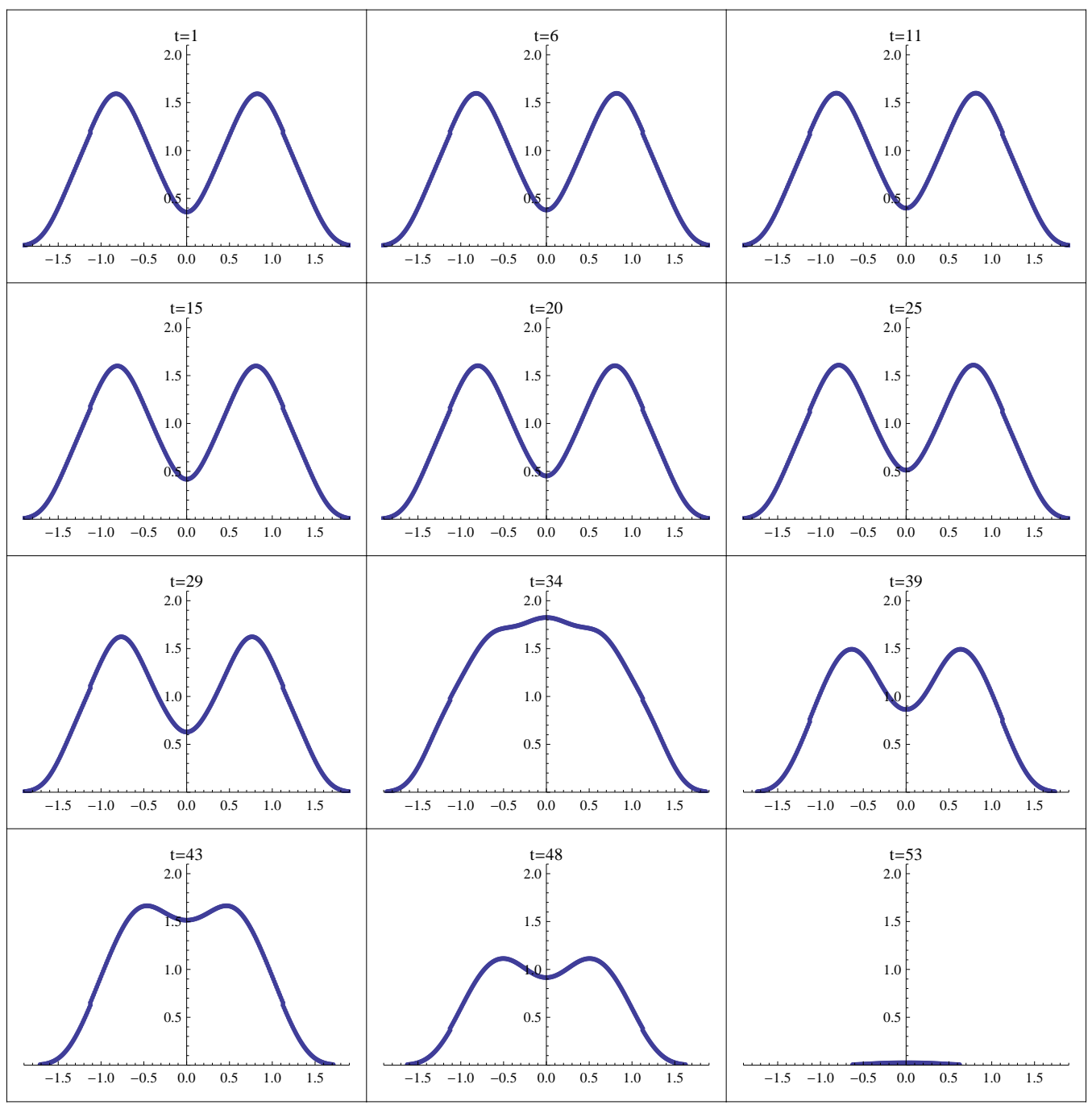

Figure 4.26: Evolution of solution ending in extinction. $w=1.65$

4.0.8.2 Two interacting period-2 equilibrium We now study the effects of two interacting patches in the case $r=2.5$ with a stable period- 2 attracting non-spreading solution. we let $u_{e 1}(x)$ and $u_{e 2}(x)$ be the stable attractor depicted in Figure 4.21, assigned so that $u_{e 1}(0)>u_{e 2}(0)$. As previously, we determine $u_{e i}(x)$ numerically by iterating until the even year solutions meet max difference fixed point stopping criteria. In this section, we will consider both a symmetric initial condition,

$$
u_{0}(x)=u_{e 1}\left(x+\frac{w}{2}\right)+u_{e 1}\left(x-\frac{w}{2}\right)
$$


and an asymmetric initial condition

$$
u_{0}(x)=u_{e 2}\left(x+\frac{w}{2}\right)+u_{e 1}\left(x-\frac{w}{2}\right) .
$$

For each case we then iterate the system until the fixed point stopping criteria is reached on the even year solutions, calling this final (even-year) state $u_{\Omega}$. For this period2 solution $\int_{-\infty}^{\infty} u_{e 1}(s) \mathrm{d} s=2.388$ and $\int_{-\infty}^{\infty} u_{e 2}(s) \mathrm{d} s=2.012$.

We first examine the case of the symmetric initial data,

$$
u_{0}(x)=u_{e 1}\left(x+\frac{w}{2}\right)+u_{e 1}\left(x-\frac{w}{2}\right)
$$

On the y-axis we plot the average of the total population for the even and odd year final state. Therefore we would expect the result to an integer multiple of $\frac{1}{2}(2.388+2.012)=$ 2.2 .

In Figure 4.27 we see final states with total populations given by $0 \times 2.2,1 \times$ 2.2, and $2 \times 2.2$ (grey dashed lines), indicating populations with $0,1,2$, or 3 patches. The final populations which are 2.2 (green) appear to be numerical artifacts, where a numerical instability slowly leads to an asymmetric density curve. For example, in Figure 4.28 we see that at about $t=134$ the asymmetry develops enough to be visually detectable. In Figure 4.29 we show an example of the initial symmetric 2-patch state collapsing to extinction. 


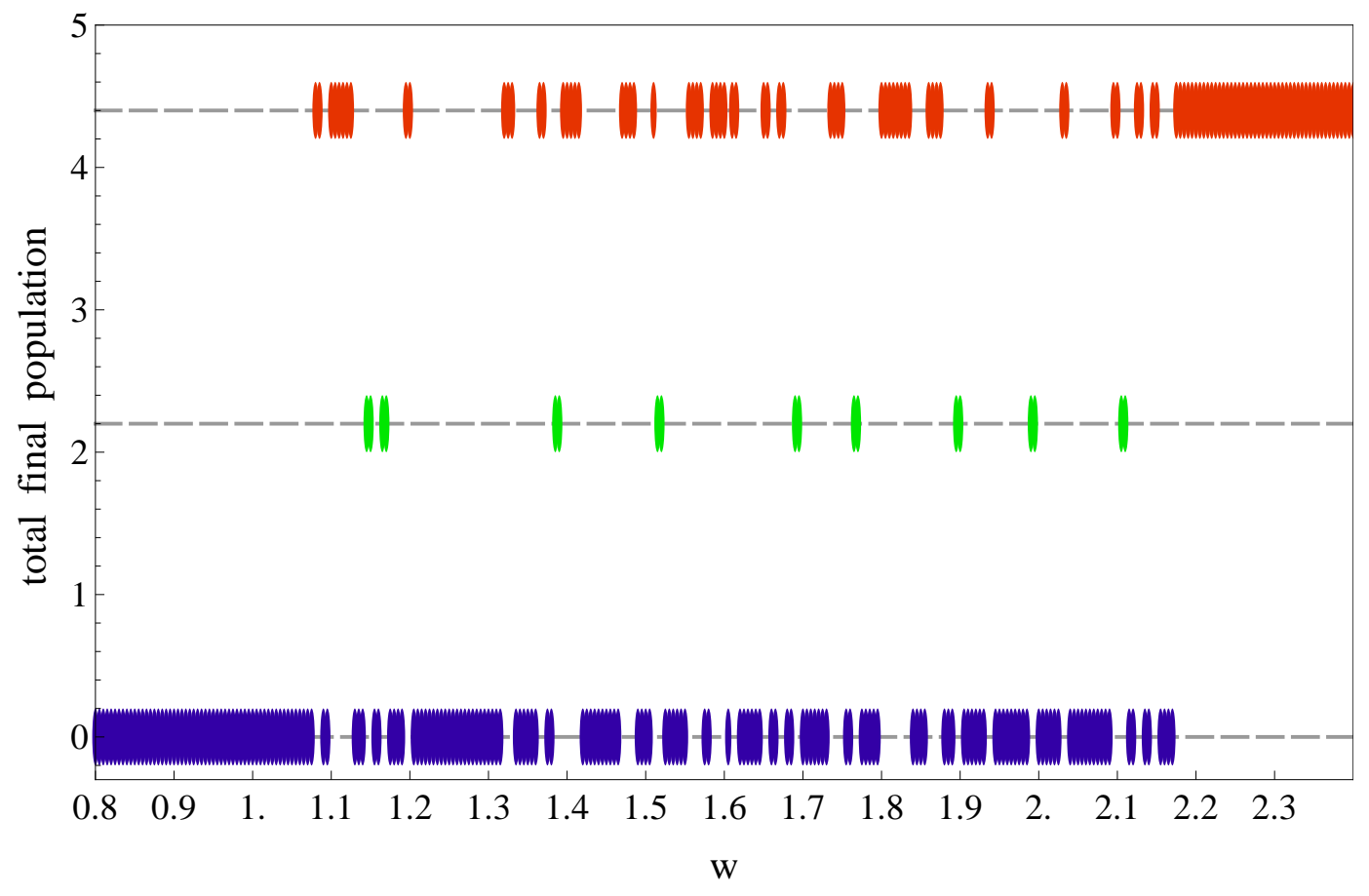

Figure 4.27: Total population of even year end-state versus spacing parameter $(w)$ for symmetric initial data. Grey dashed lines are multiples of 2.2. 


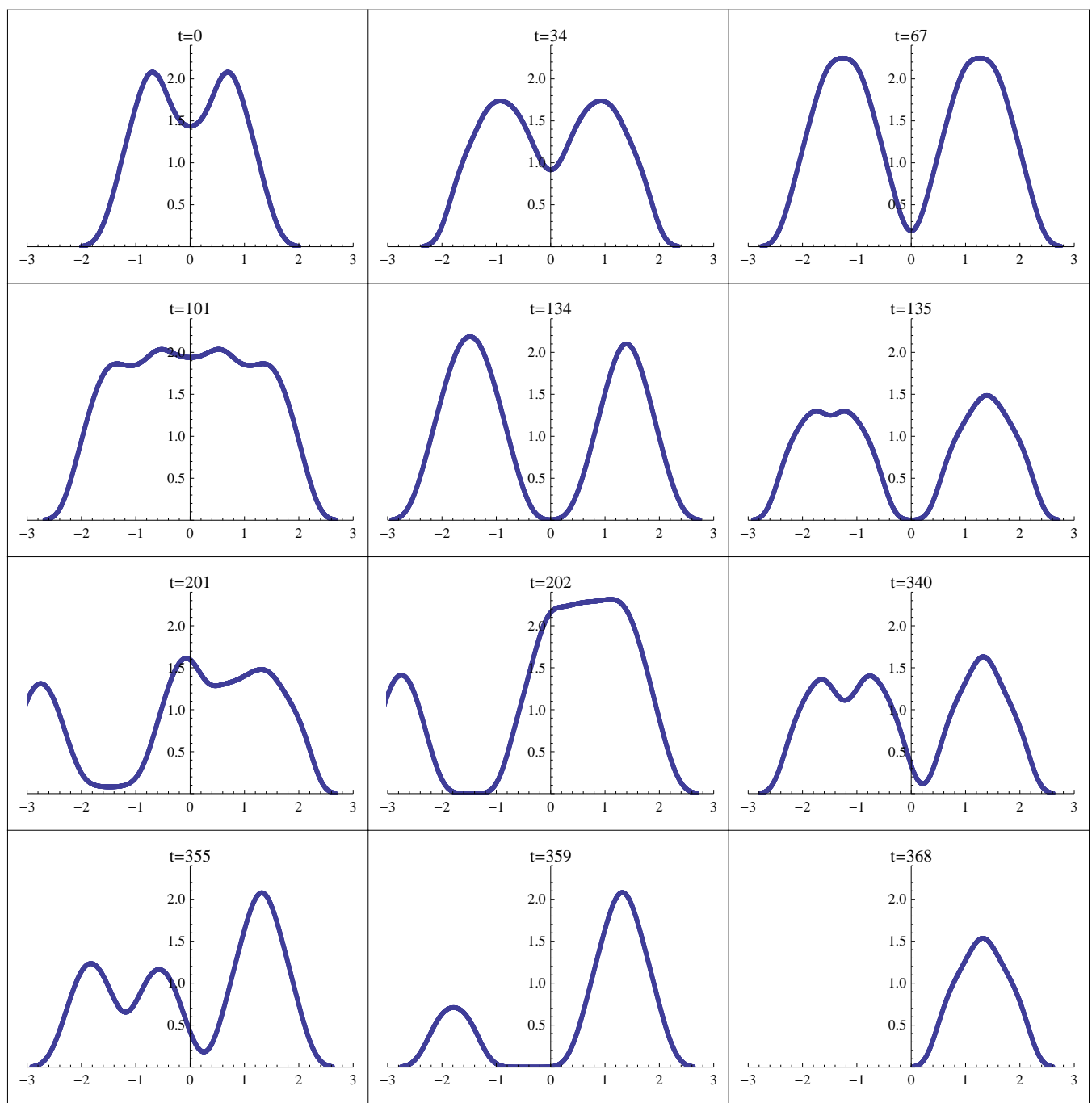

Figure 4.28: Illustration of collapse from 2-patches to one patch for symmetric initial data. $w=1.387$ 


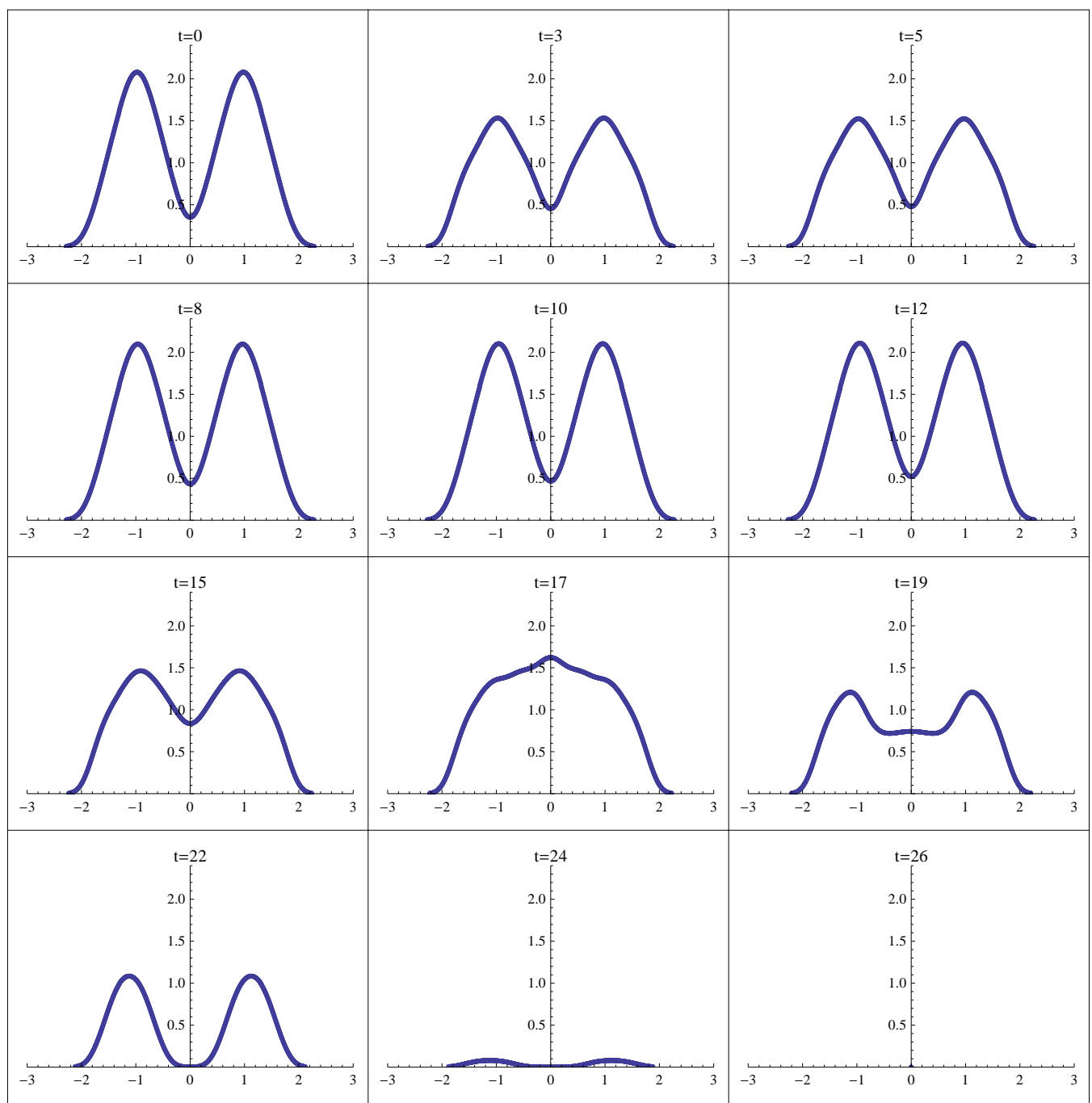

Figure 4.29: Illustration of extinction from symmetric two-patch initial data. $w=1.96$

Next we examine the case of the asymmetric initial data,

$$
u_{0}(x)=u_{e 2}\left(x+\frac{w}{2}\right)+u_{e 1}\left(x-\frac{w}{2}\right)
$$

On the $y$-axis we plot the total population of the odd year final state. Depending on the number of total final patches, and their relative phase, we would expect the final (odd or even year) population to be of the form $2.388 n_{e 1}+2.012 n_{2}$, where $n_{e 1}$ and $n_{e 2}$ are the number of $u_{e 1}$ and $u_{e 2}$ phased patches respectively.

In Figure 4.30 we see there are populations at $2.388 * 2=4.776$ and $2.012 * 2=$ 
4.024 represented by the blue-grey datum. These represent solutions where out of phase patches settle into 2 in-phase patches. In Figure 4.31 we illustrate the evolution of these dynamics.

Populations terminating at $2.388+2.012=4.4$, represented in fuchsia, represent two patches remaining in phase. Population ending in either 2.012 or 2.388 (green) represent 2 patches merging into one. The dynamics of this merger are represented in Figure 4.32. Finally in Figure 4.33 we show the dynamics of a solution collapsing to extinction.

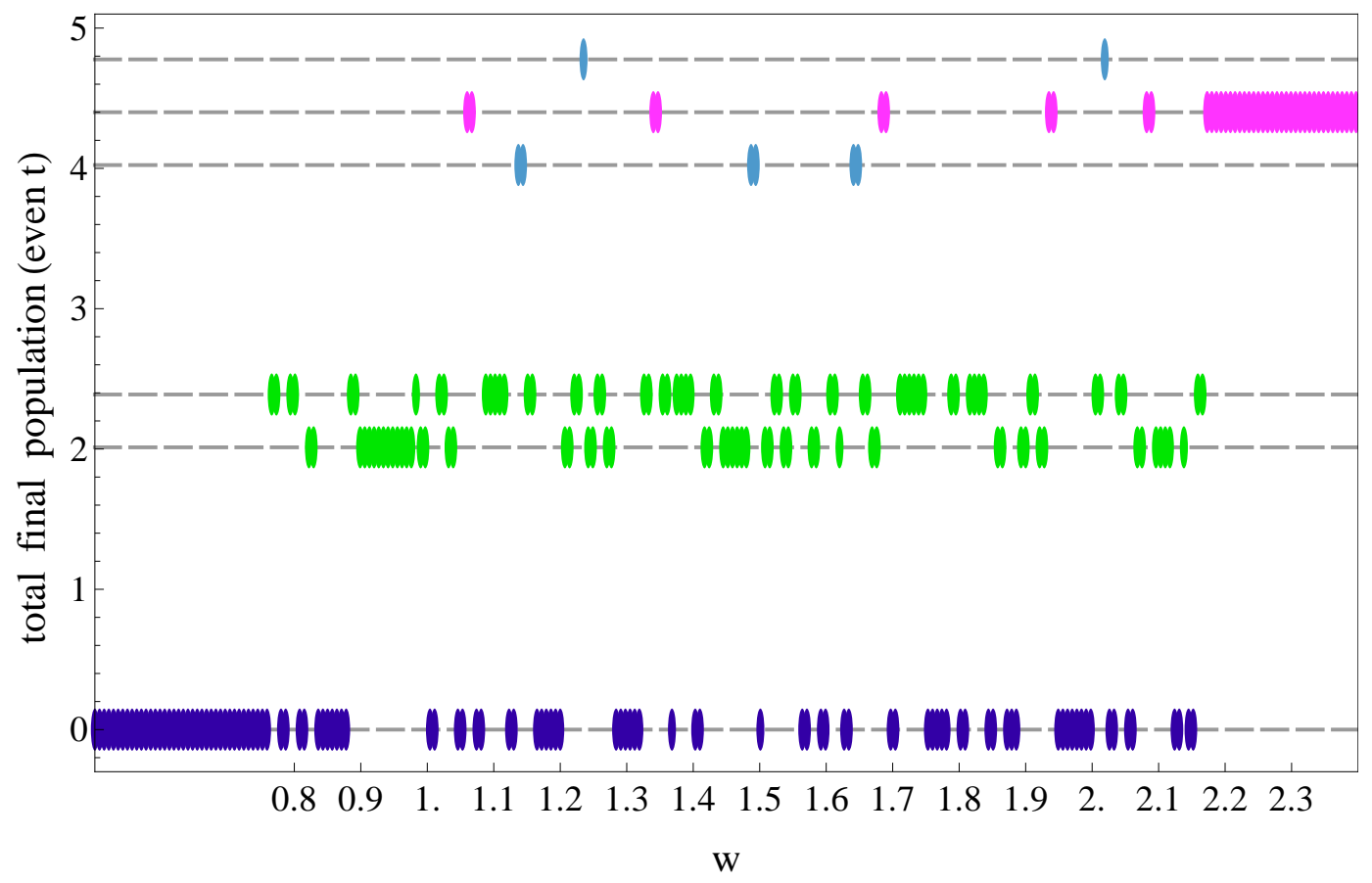

Figure 4.30: Total population of final state odd year end-state versus spacing parameter (w) for asymmetric initial data. 


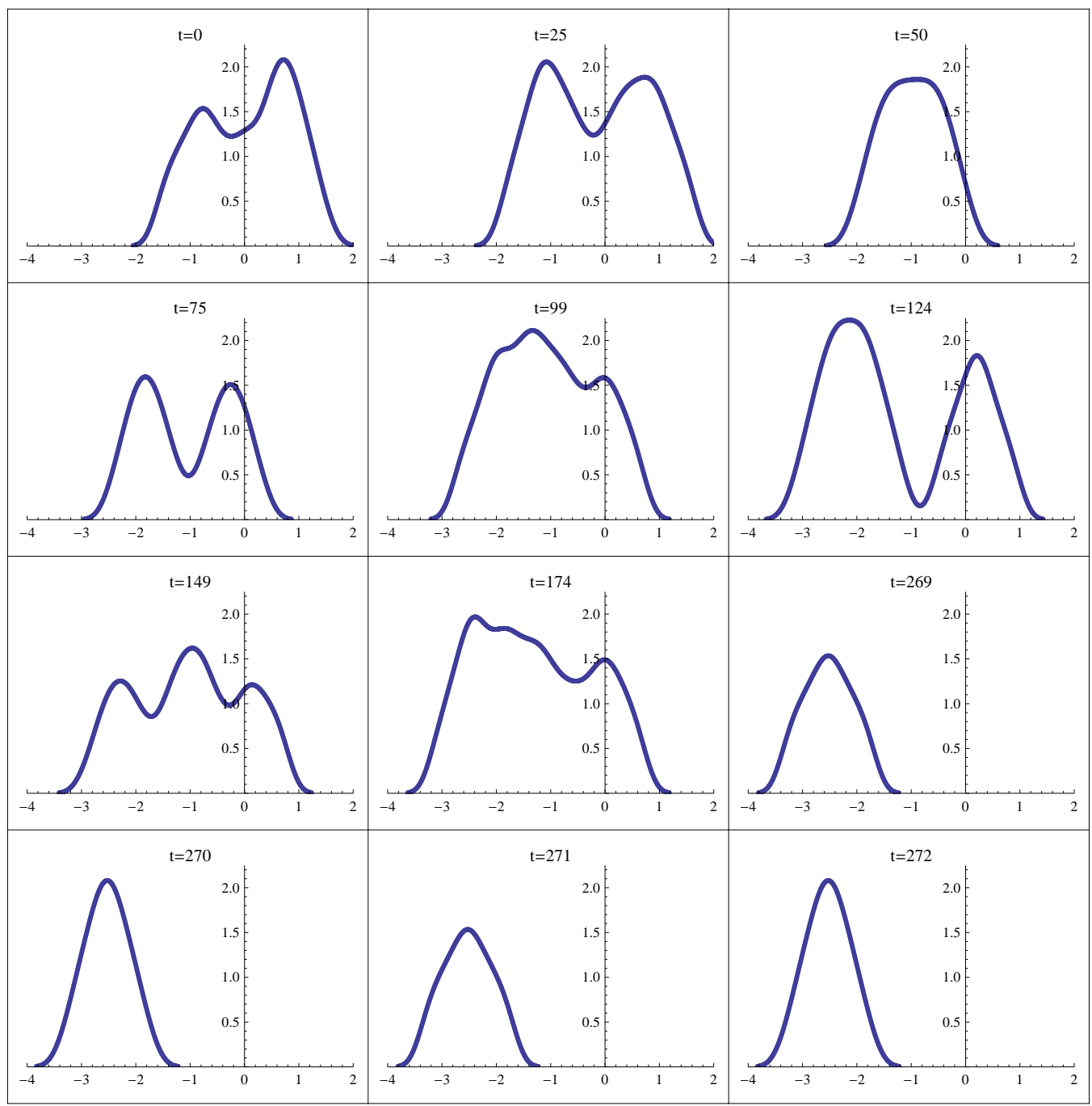

Figure 4.31: Illustration of merger from 2-patches to one patch for asymmetric initial data. $w=1.47$ 


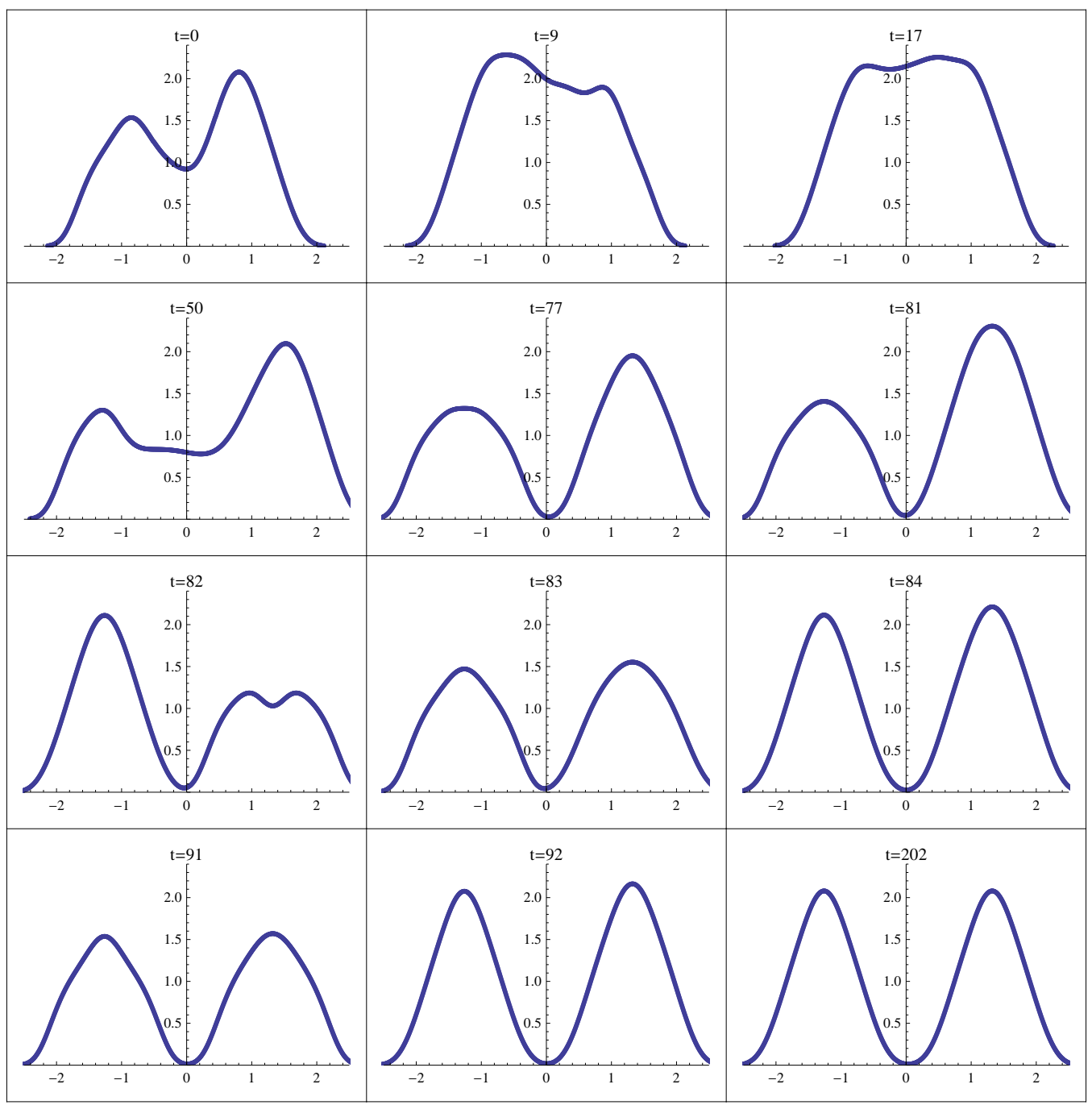

Figure 4.32: Illustration of out of phase 2 patch initial data, forming into an in-phase 2 patch oscillation. $w=1.646$ 


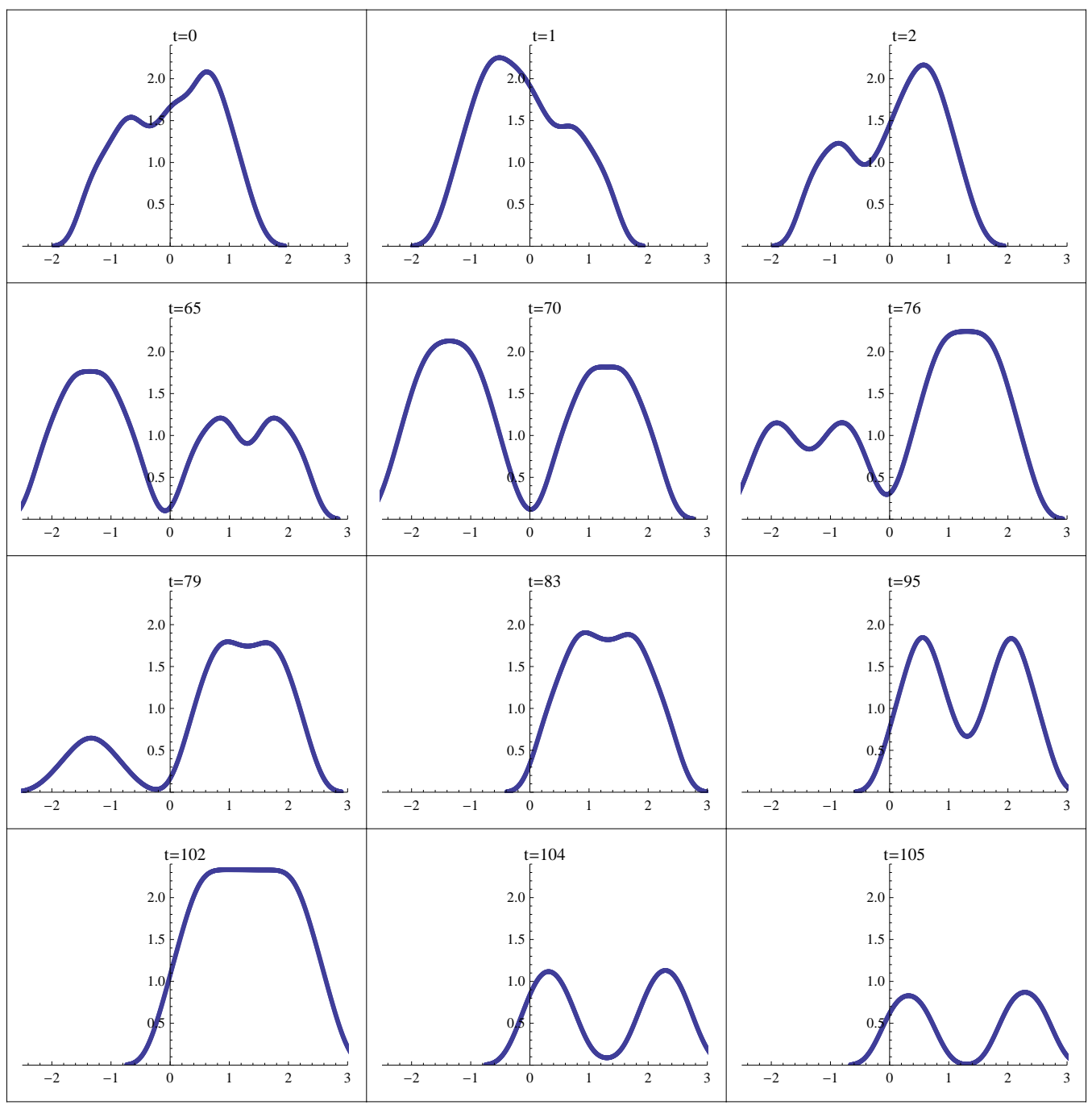

Figure 4.33: Illustration of extinction from asymmetric two-patch initial data. $w=1.3$

\subsubsection{Patch formation over large perturbed initial domain with single sta-}

ble equilibria We now wish to consider the evolution when the support of the initial data is much larger then that of a single non-spreading solution. We will use the growth function in Figure 4.20 which has a single asymptotically stable non-spreading solution (depicted in Figure 4.22). Since the length scale of the equilibrium is roughly 2 length units, we will initiate over an interval of length much larger then 2 , and let the density be super-Allee threshold with small fluctuations over length-scales of $\sigma$. To accomplish this we will use a piecewise constant function, constant over sub-intervals of length $\sigma$, 
whose height on each sub-interval is given by a random variate drawn from the uniform distribution on $(1.1,1.2)$. The functional form is thus

$$
u_{0}(x)= \begin{cases}0, & |x|>n \sigma \\ u_{\{-n\}}, & -n \sigma<x<(-n+1) \sigma \\ u_{\{-n+1\}}, & (-n+1) \sigma<x<(-n+2) \sigma \\ \ddots & \ddots \\ u_{\{n-2\}}, & (n-2) \sigma<x<(n-1) \sigma \\ u_{\{n-1\}}, & (n-1) \sigma<x<n \sigma\end{cases}
$$

where $u_{\{i\}}$ are i.i.d. unif $(1.1,1.2)$ random variates.

We then consider cases where $\sigma>>2, \sigma=2$, and $\sigma<<2$ while holding the initial support to be $(-40,40)$.

As can be seen in Figures 4.34-4.36, stable non-interacting patches emerge from the initial data after sufficient time. As can be seen in Figure 4.34, when the scale of variation in the initial data in long compared to the size of the equilibrium, stable patches are sparse and tend to occur only near places of relatively large fluctuations in the initial data such as near $x=-36, x=-30$ for instance. In Figure 4.35 with $\sigma=2$ we see the density of stable patches is much higher then in the $\sigma=6$ case with patch formations till tending to occur near places sharp jumps in initial density. In the case of small $\sigma$ in Figure 4.36 we see the total patch formation density is not significantly different form that in Figure 4.35 suggesting saturation. The pattern of where stable patches will form is not readily discernable. 


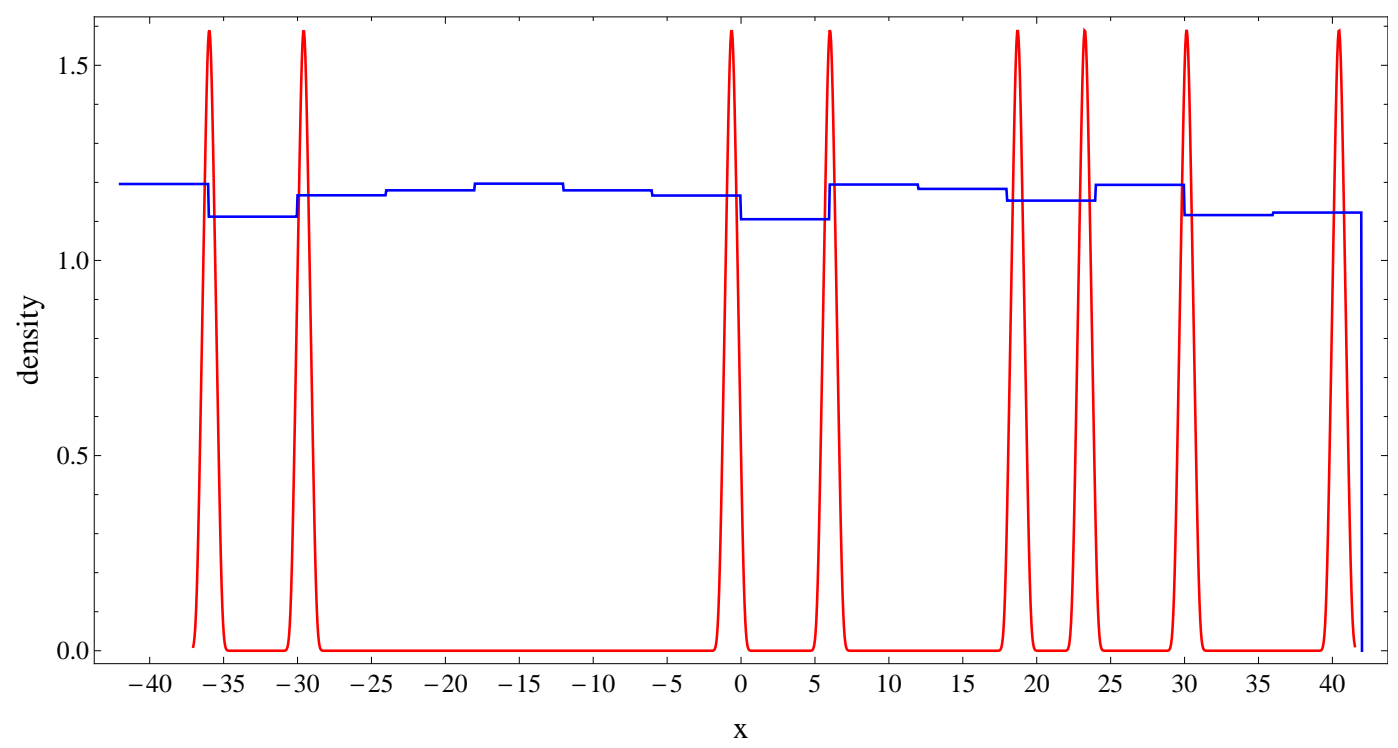

Figure 4.34: Pattern formation with $\sigma=6$. Initial data is shown in blue, iterations $110-$ 120 are shown in red.

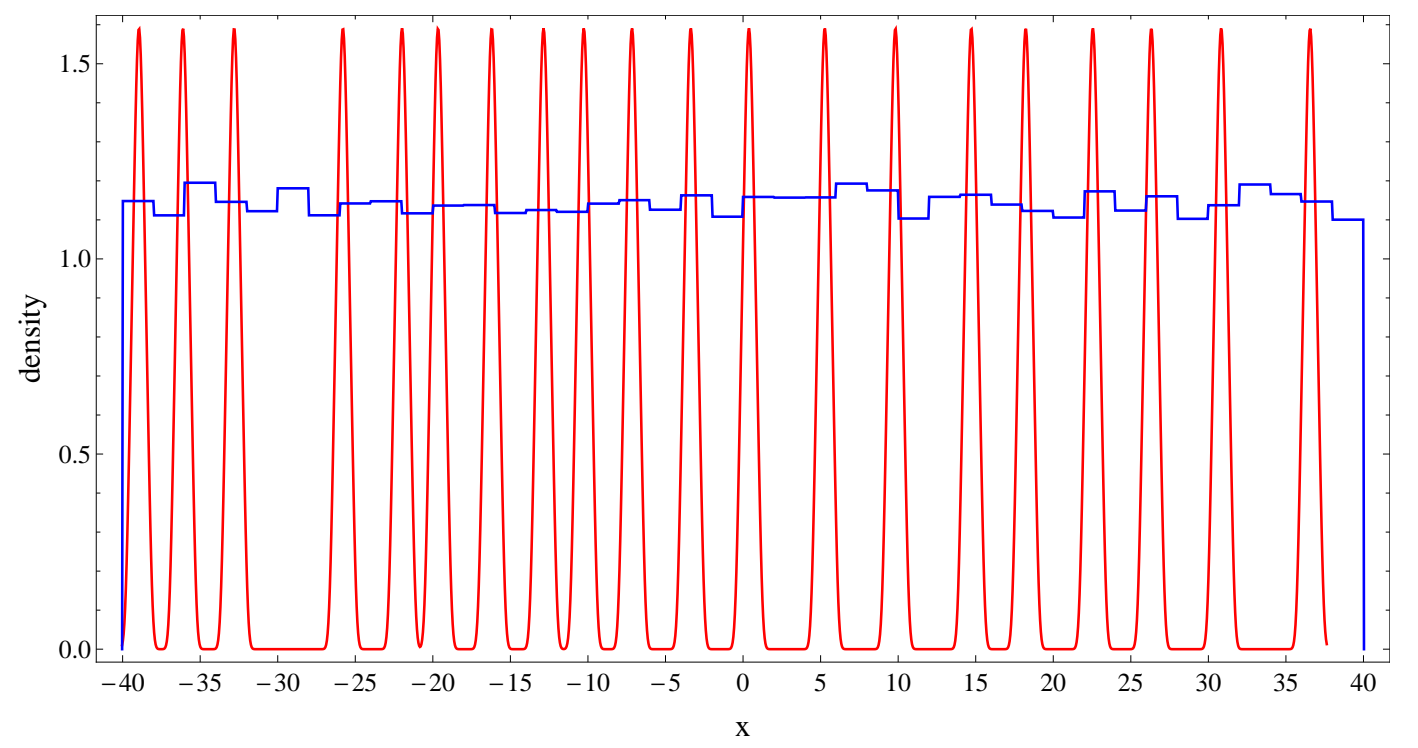

Figure 4.35: Pattern formation with $\sigma=2$. Initial data is shown in blue, iterations $110-$ 120 are shown in red. 


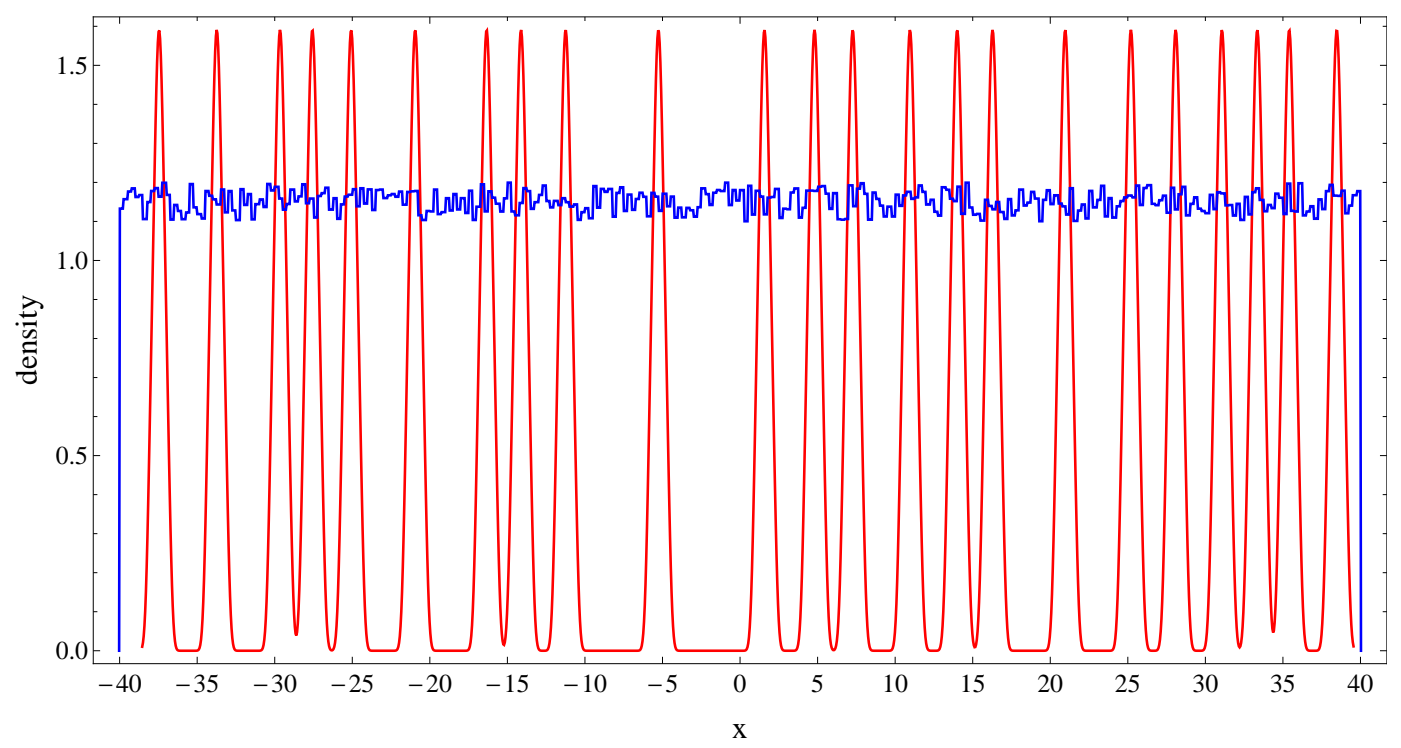

Figure 4.36: Pattern formation with $\sigma=0.25$. Initial data is shown in blue, iterations $110-120$ are shown in red.

\subsubsection{Patch formation over large perturbed initial domain with period-2}

equilibria We now consider a circumstance similar to that in Section 4.0.8.1, however we will use the growth function $g(u)=u \exp (2.5(1-u))\left(\frac{\left(1+1.3^{7}\right)\left(\frac{u}{1.3}\right)^{7}}{1+\left(\frac{u}{1.3}\right)^{7}}\right)$ which has a stable period-2 orbit, as demonstrated in Figure 4.21.

We find results very similar to those from Section 4.0.8.3, where after sufficient time, we are left with a sequence of essentially non-interacting period-2 equilibria. In Figures 4.37-4.39, (b) we see the emergent final stable solutions. The transients take a much longer time to evolve then they did in case of 4.0.8.3. For example, with $\sigma=0.25$, it took over roughly 400 iterations for the transients to dampen, while only taking about 100 for the same case in 4.0.8.3. 


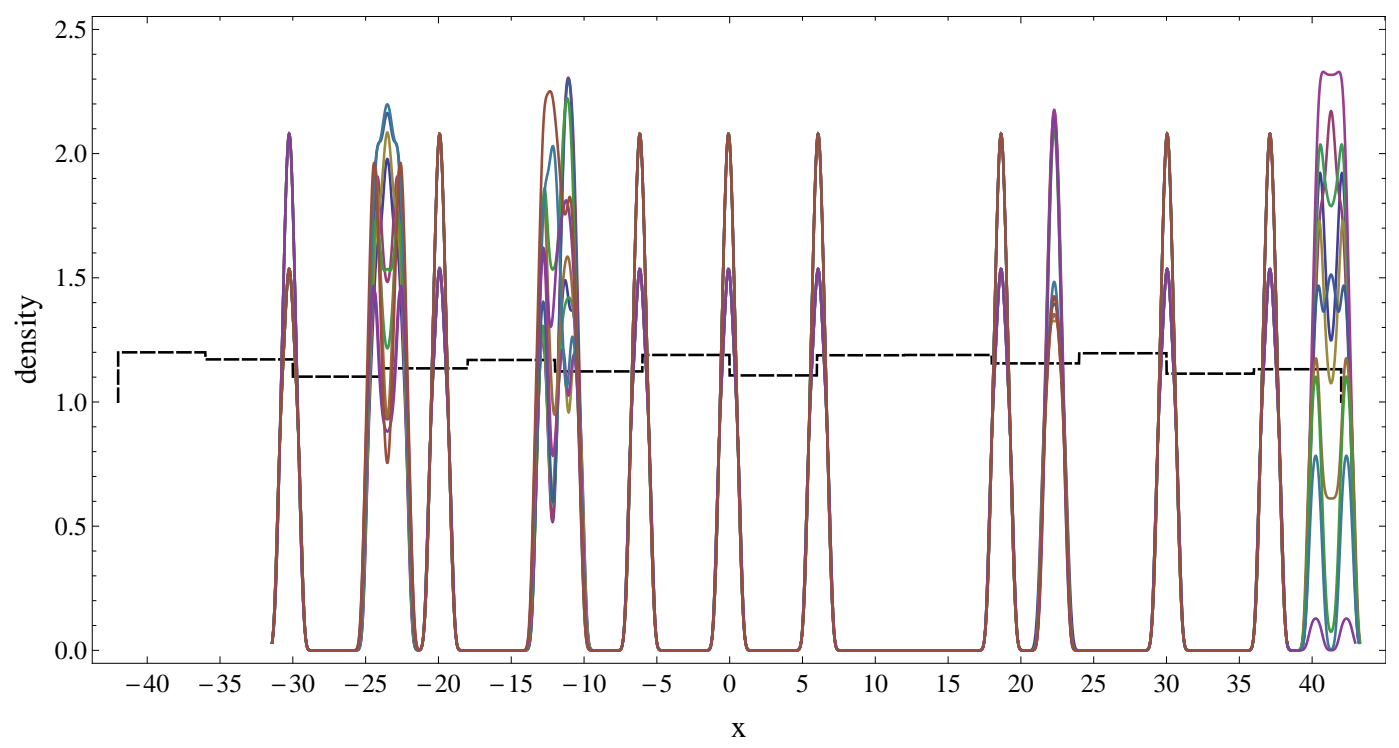

(a) $t=120, \ldots, 130$

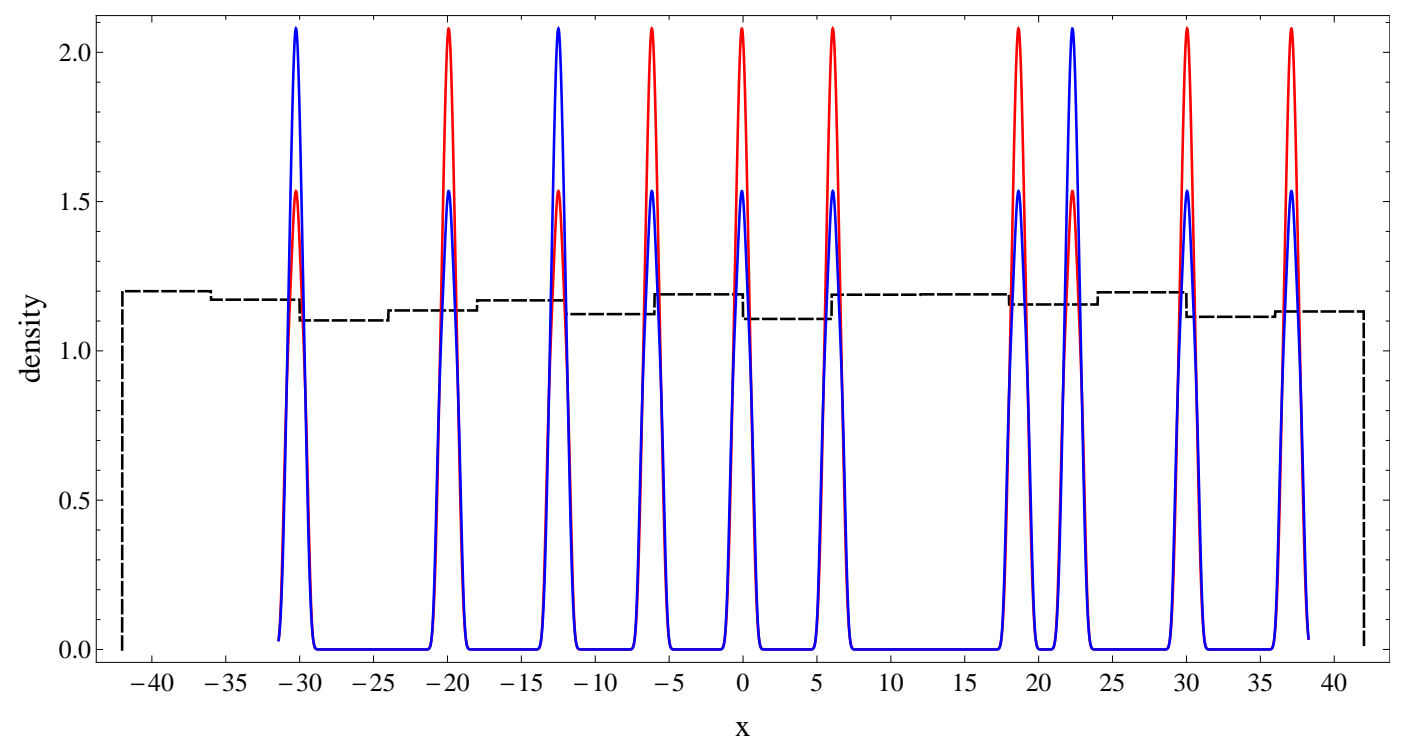

(b) $t=210, \ldots, 220$

Figure 4.37: Pattern formation with $\sigma=6$. Sub-figure (a) depicts transient phenomena, while sub-figure (b) shows the terminal state. In (b) odd-years are depicted in blue, and even-years are red. 


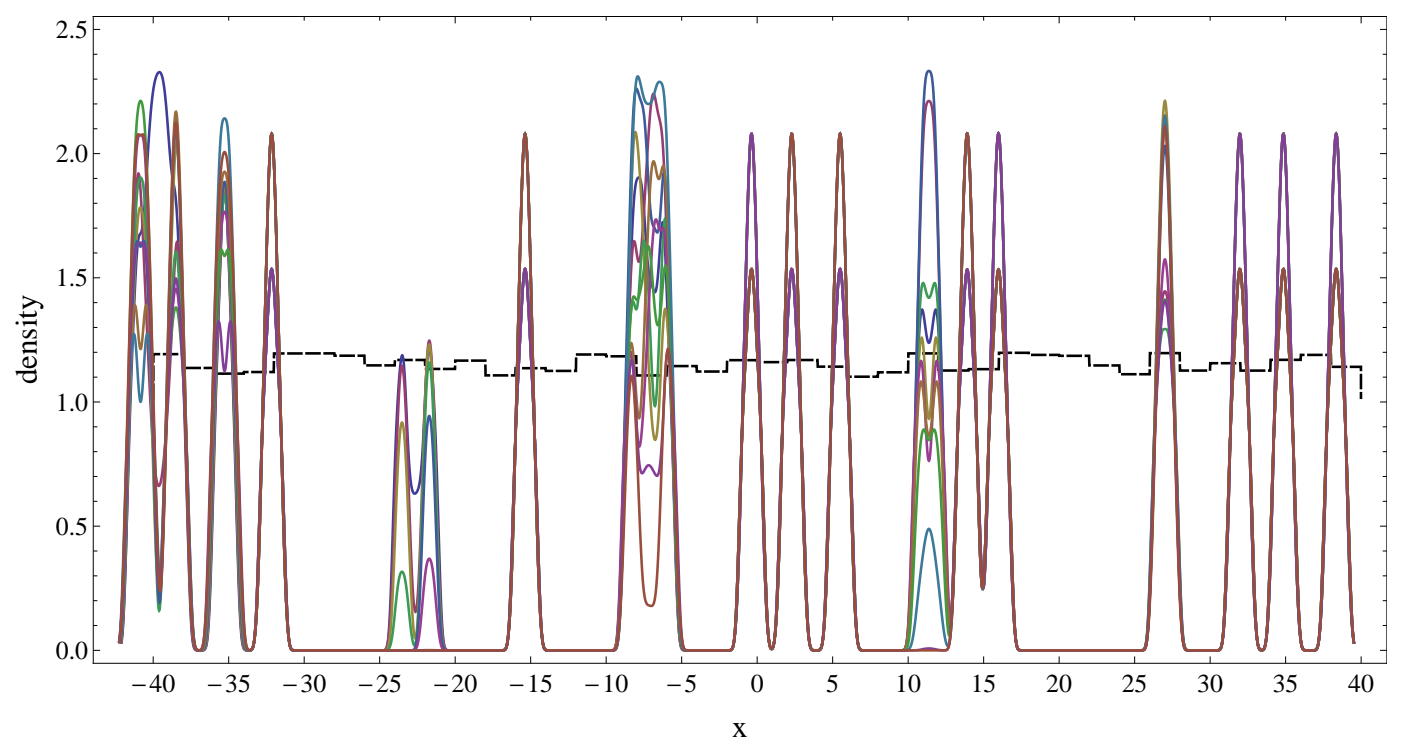

(a) $t=120, \ldots, 130$

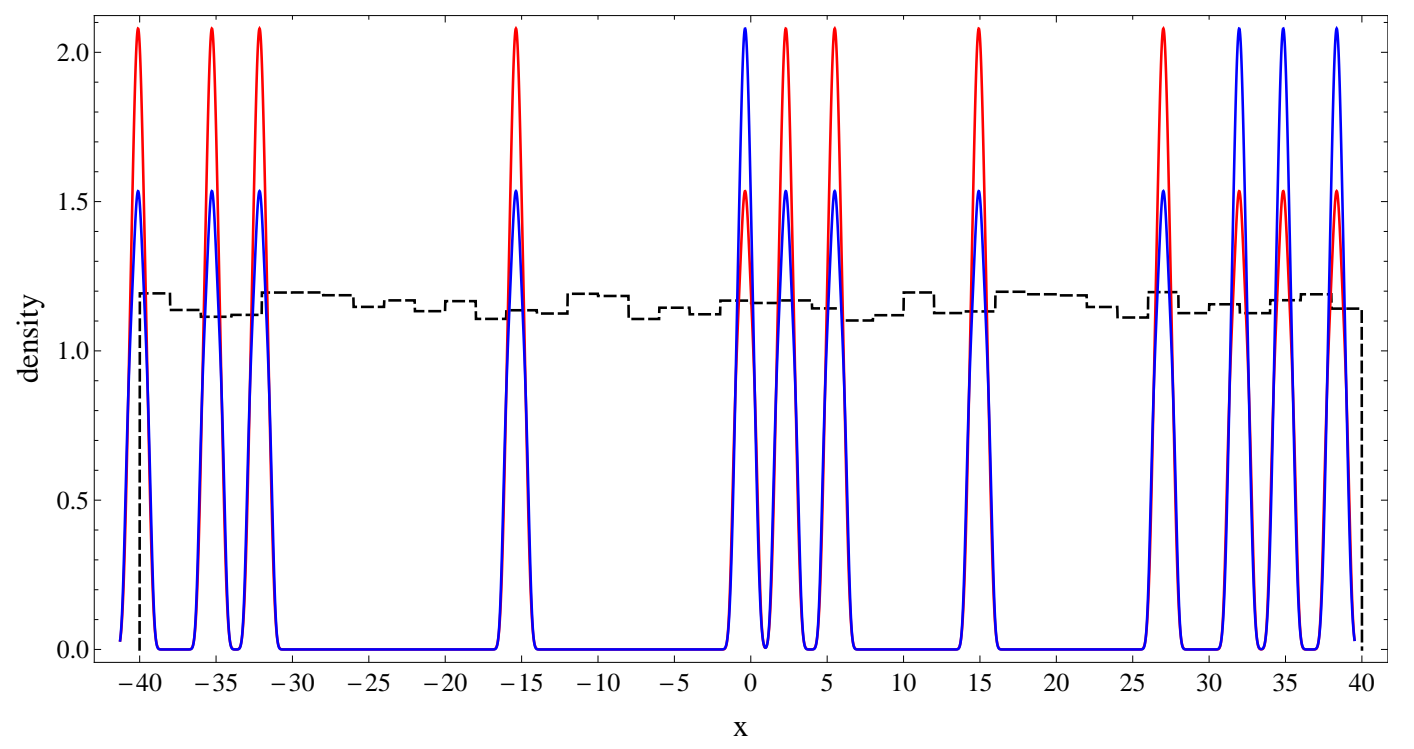

(b) $t=386, \ldots, 396$

Figure 4.38: Pattern formation with $\sigma=2$. Sub-figure (a) depicts transient phenomena, while sub-figure (b) shows the terminal state. In (b) odd-years are depicted in blue, and even-years are red. 


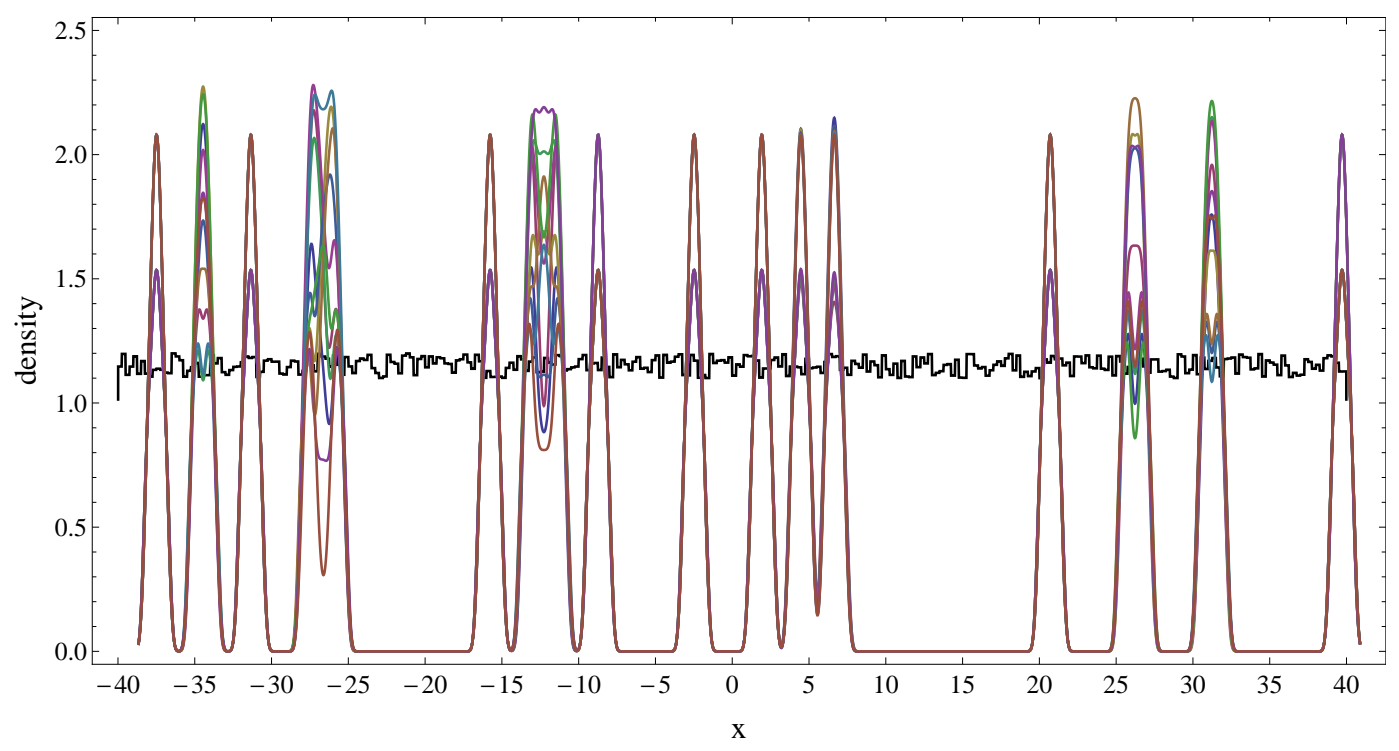

(a) $t=250, \ldots, 260$

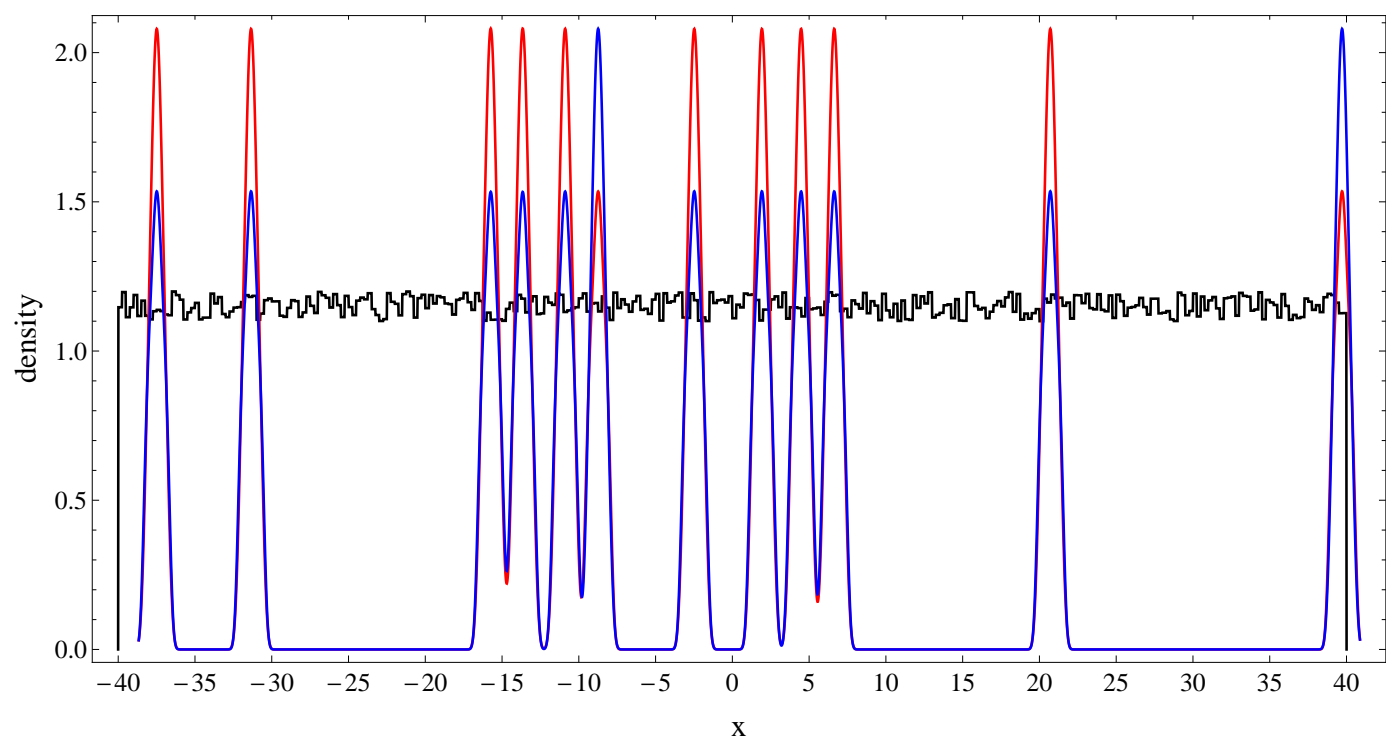

(b) $t=590, \ldots, 600$

Figure 4.39: Pattern formation with $\sigma=2$. Sub-figure (a) depicts transient phenomena, while sub-figure (b) shows the terminal state. In (b) odd-years are depicted in blue, and even-years are red. 


\subsubsection{Spread with essential extinction}

In the context of non-spatial recursive population dynamics $\left(u_{n+1}=g\left(u_{n}\right)\right)$, growth functions with strong-Allee effect and a particularly virulent form of overcompensation are said to exhibit essential. Essential extinction occurs when high output densities are subsequently mapped to sub-Allee threshold densities. More formally, if $u_{\text {allee }}$ is the Allee-threshold of $g(u)$, and $u_{\max }=\max _{u \geq 0} g(u)$ then $g(u)$ has essential-extinction if $g\left(u_{\max }\right)<u_{\text {allee }}$

It is well known in non-spatial dynamics (Gyllenberg 1996, Schrieber 2001) that growth functions exhibiting essential extinction will face extinction for almost-every initial condition, where of course "almost-every" is used in the measure theoretic sense. The only condition that need apply is that $g(u)$ be smooth, and the Schwartzian derivative, defined by

$$
\frac{g^{\prime \prime \prime}(u)}{g^{\prime}(u)}-\frac{3}{2}\left(\frac{g^{\prime \prime}(u)}{g^{\prime}(u)}\right)^{2},
$$

be negative for all $u>u_{\text {allee }}$. This condition is found to hold for most commonly studied growth functions, however uni-modal growth functions can be constructed which violate the negative Schwartzian hypothesis while simultaneously possessing essential extinction and a positive stable-equilibrium (Schreiber 2001). We will numerically demonstrate that its possible, when coupled with spatial dispersion, for growth functions with essential extinction that satisfy the Schwartziann hypothesis to have spatial spread and unbounded population growth.

Using the growth function mentioned in Section 4.0.7.2, with $r=2.3$, we let

$$
g(u)=u \exp (2.3(1-u))\left(\frac{\left(1+1.3^{7}\right)\left(\frac{u}{1.3}\right)^{7}}{1+\left(\frac{u}{1.3}\right)^{7}}\right) .
$$

It can be verified graphically that $g$ satisfies the Schwartzian hypothesis. 


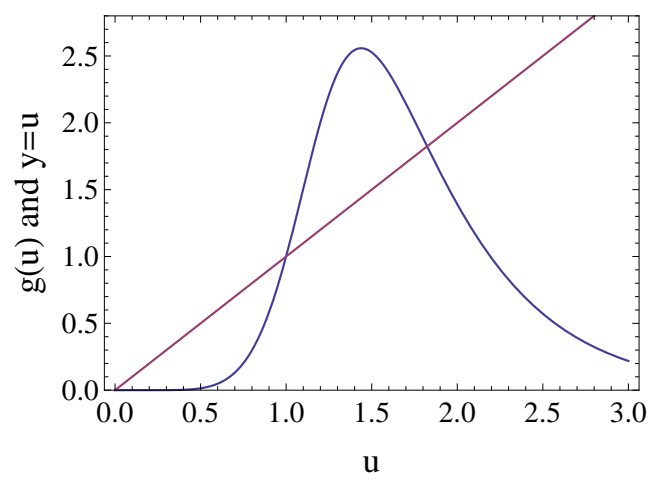

Figure 4.40: The Hill-Ricker growth function used for this simulation.

Similarly, as throughout Section 4.0.7, we will use the kernel

$$
k(x)=1.1109 \exp \left(-\left(\frac{x}{0.4965}\right)^{4}\right) .
$$

Also as in Section 4.0.7 we use the initial data

$$
u_{0}(x)= \begin{cases}1.25, & |x|<0.5 \\ 0, & |x| \geq 0.5 .\end{cases}
$$

We then iterate the equation for 4000 generations, and for each generation determine the domain size and total population. For the purposes of this Section the domain size of $u_{i}$ is defined as

$$
\sup \left\{x \mid u_{i}(x)>0.1\right\}-\inf \left\{x \mid u_{i}(x)>0.1\right\},
$$

or in other words the length of the interval from where $u_{i}(x)$ first rises above 0.1 to where it falls below 0.1 for the final time.

The total populations of $u_{i}(x)$ is of course defined as

$$
\text { tot. pop. }=\int_{-\infty}^{\infty} u_{i}(x) \mathrm{d} x .
$$

In Figure 4.41 we sample of the progression of density vs. $x$ for several different starting times. A general trend that can be seen is that the solutions spread, and as time 
progresses the density becomes more oscillatory. In some loose sense however we see that the length-scale of the oscillations or "finger"-like regions is still on the order of a few length units regardless of the generation. An important dynamical feature can be seen for $t=299,300,301$ near $x=-7$. From $t=299$ to $t=300$ we see the relatively wide patch from $-9<x<-5$ grow to near the maximum value of $g(u), 2.5$ (see Figure 4.40), at which point the region splits into 2 "fingers" due to strong overcompensation. This splitting dynamic appears to drive the oscillatory behavior. 


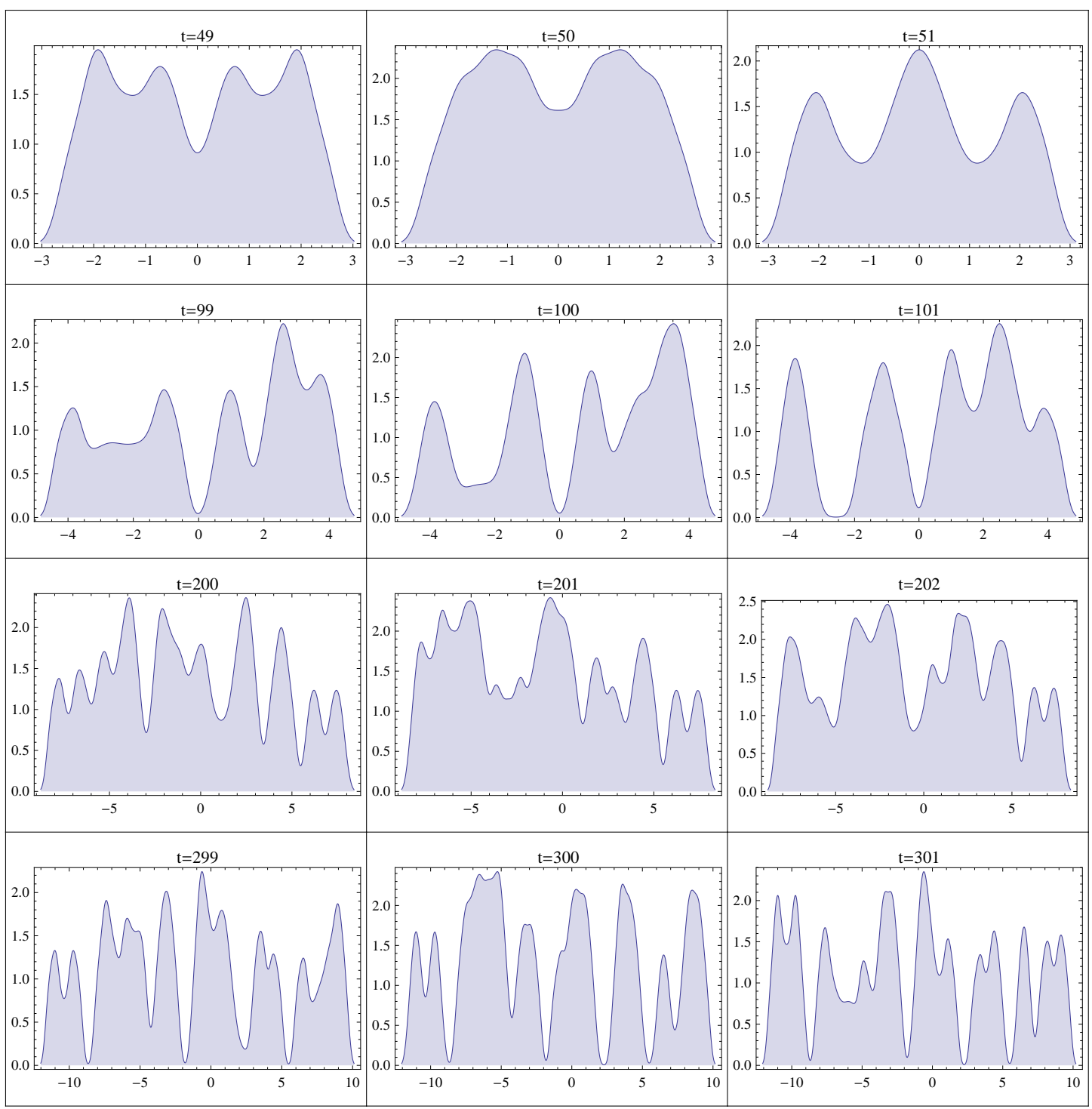

Figure 4.41: A sample of the density $u_{t}(x)$ vs. $x$ for several different generations when $r=$ 2.3. Note immediate successors are shown across the columns, and large time differences across rows. Also note the $x$-scale varies across rows.

In Figure 4.42 we plot the total population and domain size as a function of time. It can clearly be seen that the long term trend is that both are increasing linearly with time, with some (deterministic) stochastic variation. The blue data points, which are the total population clearly exhibit a spreading fan shape, while the domain length appears as straight line with some jaggedness. 


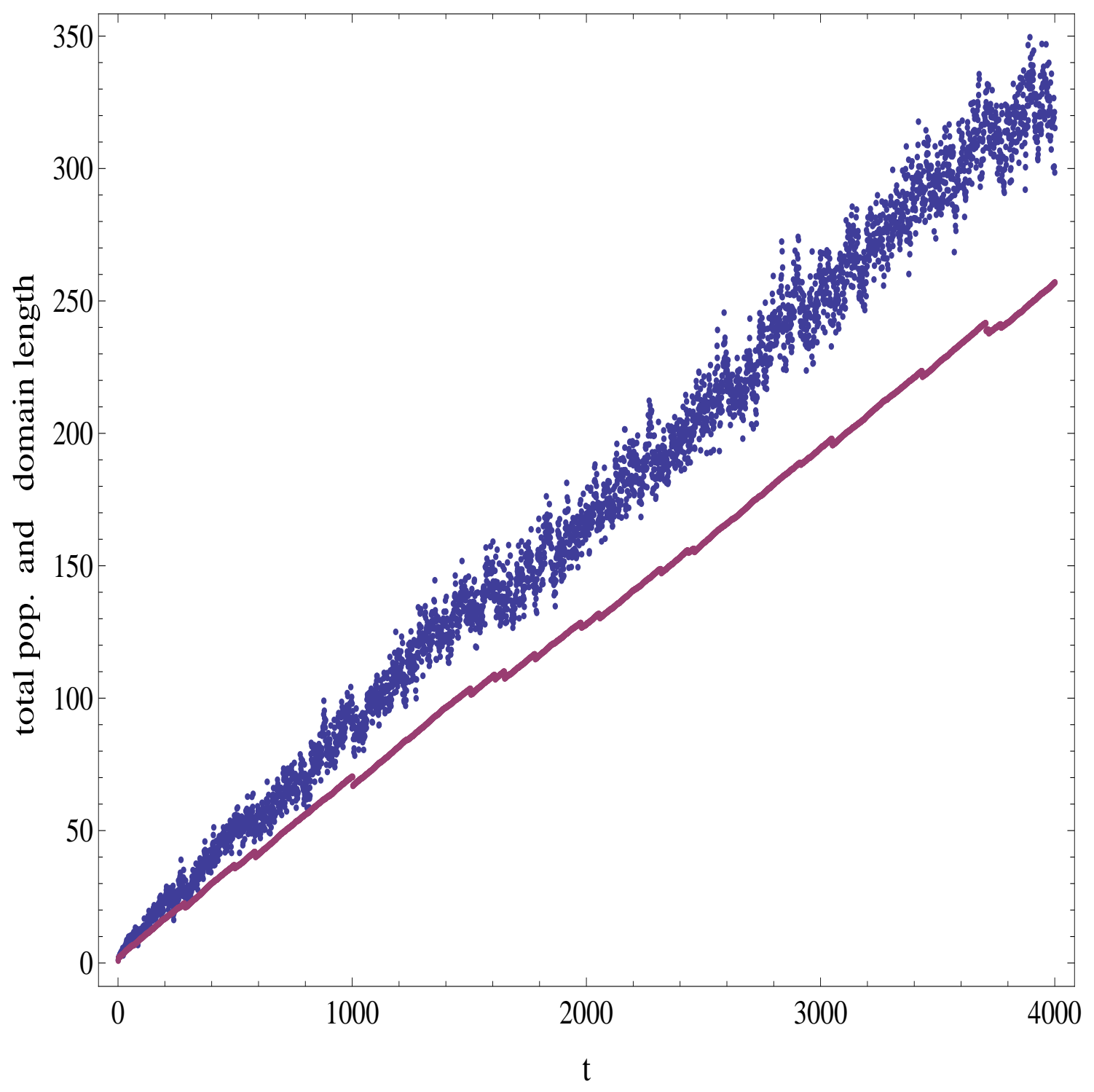

Figure 4.42: A plot of the total population and domain size vs. time. Total population is blue, and domain size is violet.

To explain why the total population is fan shaped, we first note that this is consistent with a process whereby the variance of the increment $(\operatorname{pop}(t+1)-\operatorname{pop}(t))$ is increasing. In fact when a linear regression is performed and the accumulated square residuals are plotted, the accumulated square residuals are fitted very well by a quadratic. This indicates the variance of the increment increases linearly (the derivative of the quadratic). We then perform a iterative procedure where a regression is performed on total popu- 
lation, the increment variances are determined by fitting a quadratic to the accumulated square residuals, whereby a new weighted regression is performed using these variances, until a fixed point is reached. Using this procedure we determined the regression lines for the total population, and variance of increment are

$$
\begin{aligned}
\operatorname{pop}(t) & =5.518+0.0825 t \\
\text { variance of increment }(t) & =14.35+0.0278 t .
\end{aligned}
$$

In Figure 4.43 we plot the regression line versus the data, and plot the integral of the variance of increment versus the accumulated total square residuals. 


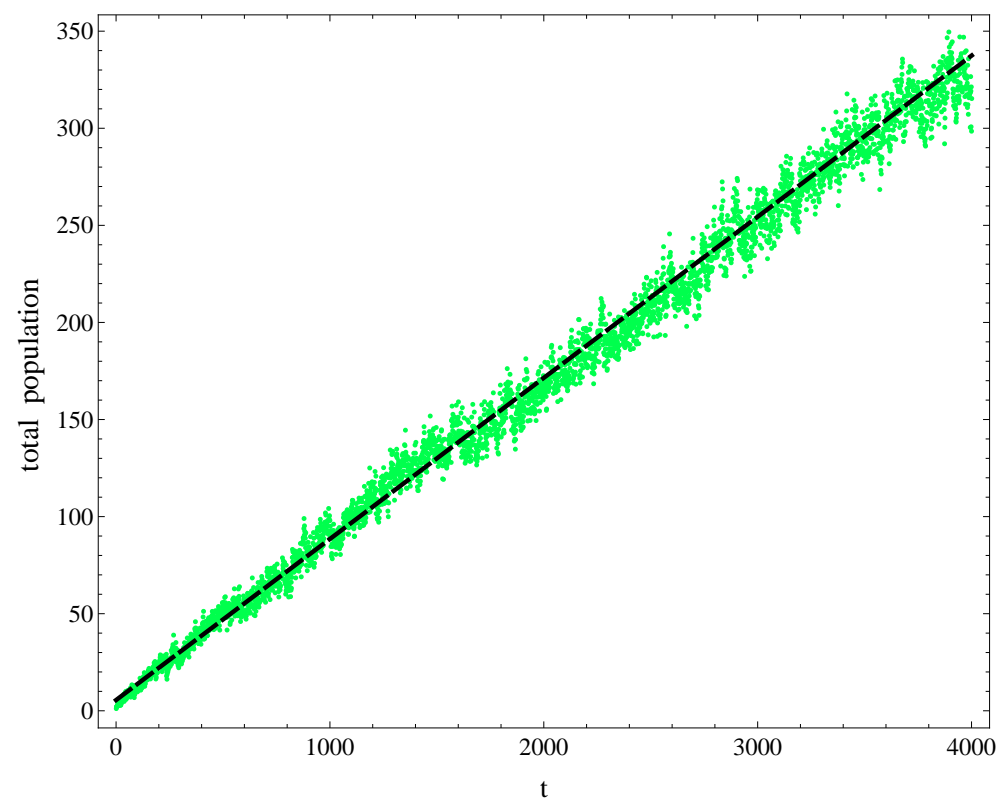

(a) total population vs. $t$

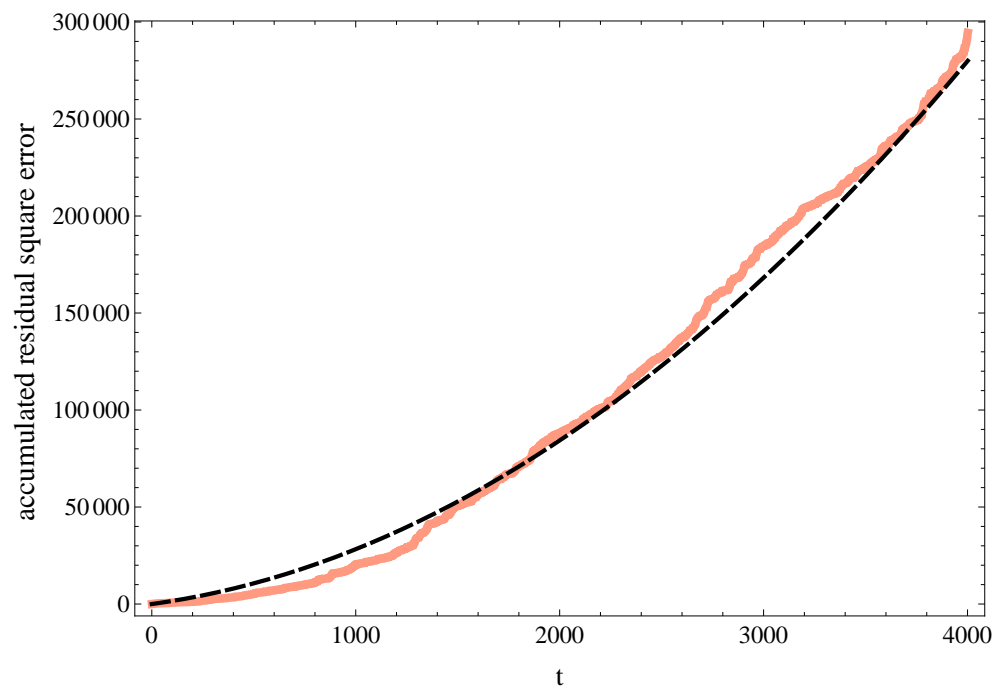

(b) accumulated squared residuals vs. $t$

Figure 4.43: Sub-figure (a) is plot of total population together with the regression line from Eq. 4.4. Sub-figure (b) is total accumulated residual square error, together with the fitted quadratic, $14.35 t+0.0139 t^{2}$.

To understand why the variance of increment increases linearly, it is reasonable to speculate that there is some de-correlation length scale for $u_{i}(x), \ell$, and whereby $u_{i}(x+\ell)$ would be statistically independent from $u_{i}(x)$. If the total population change on each 
populated patch of length $\ell$ is a random variable with mean zero and variance $\sigma_{\{\text {pop }, \ell\}}^{2}$, and the total domain length is $L_{\mathrm{dom}}$, then the change in total population can be considered the sum of approximately $\frac{L_{\mathrm{dom}}}{\ell}$ independent identically distributed random variables of mean zero and variance $\sigma_{\{\text {pop }, \ell\}}^{2}$. Thus neglecting the contributions from the edge of the domain where spreading is occurring, which will be negligible for large domains, we see the variance in change in total population will be $\frac{L_{\mathrm{dom}}}{\ell} \sigma_{\{\mathrm{pop}, \ell\}}^{2}$. Since $L_{\mathrm{dom}}$ is increasing linearly, we would therefore expect the slope of the variance of increment (Eq. 4.4) to be well approximated by $\frac{L_{\mathrm{dom}}^{\prime}}{\ell} \sigma_{\{\mathrm{pop}, \ell\}}^{2}$. We will try to determine these quantities independently from simulation data and compare them to the slope of the variance of increment line, 0.0278 .

To demonstrate the existence of a de-correlation length and dtermine its value, we take random sample of size 10,000 of the form $\left\{u_{t}\left(x_{i}\right), u_{t}\left(x_{i}+s\right)\right\}$ where $s$ is fixed , $x_{i}$ is chosen randomly from populated domain and $t$ is chosen randomly from $900, \ldots, 1200$. The sample covariance is then computed. Recall the sample covariance of two generic random variables, $\left\{y_{i}, z_{i}\right\}$ is

$$
\text { S.C. }=\frac{1}{n-1} \sum_{i=1}^{n}\left(y_{i}-\bar{y}\right)\left(z_{i}-\bar{z}\right) \text {. }
$$

For large sample sizes where the Central Limit Theorem is applicable if $x_{i}$ and $z_{i}$ are i.i.d then it can be shown that S.C. will be normally distributed with mean zero and Variance $\frac{\sigma^{4}}{n}, N\left(0, \frac{\sigma^{4}}{n}\right)$, where $\sigma^{2}$ is the variance of $x$ and $y$. Thus if S.C. lies far out on the tails of the $N\left(0, \frac{\sigma^{4}}{n}\right)$ distribution, it is reasonable to assume a non-zero covariance. To apply this test to our $\left\{u_{t}\left(x_{i}\right), u_{t}\left(x_{i}+s\right)\right\}$ sample we must determine $\sigma_{u}^{2}$. Taking all the $u$ values on all our mesh points in the domain from $t=900, \ldots, 1100$ we obtain 734,054 density values which are depicted in the histogram in Figure 4.44-(a). We find $\mu_{u}=1.306$ and $\sigma_{d}^{2}=0.4272$. If we wish to consider covariances significant to the $99 \%$ confidence level, the criteria to be a statistically significant covariance becomes $\mid$ S.C. $\mid>2.56 \frac{\sigma_{u}^{2}}{\sqrt{10000}}=$ 0.0110. If Figure 4.45-(b) we plot the base-10 Log of S.C. versus length $(s)$ from $s=.01$ 
to $s=2.5$ in increments of 0.01 . The red dashed line is $\log _{10}(0.011)$ so points below it may be considered un-correlated. We see there is a significant dip at $s=1.1$, however this is where S.C. crosses from positive to negative (see Figure 4.44-(a)), and therefore should not be considered the de-correlation length. Starting at about $s=1.85$ we see S.C. remains below the significance threshold, thus it is reasonable to set the de-correlation length as $\ell=1.85$.

Finally to to generate the $i$ 'th data point so as to determine $\sigma_{\{\text {pop }, \ell\}}^{2}$ we choose a equally weighted random time from $900<t_{i}<1100$. For the domain of $u_{t}$, we randomly pick (uniform weighting) an $x_{i}$ that is not within $\ell$ of right endpoint of the domain. We then calculate the yearly change in population for interval,

$$
\int_{x_{i}}^{x_{i}+\ell} u_{t+1}(s)-u_{t}(s) \mathrm{d} s .
$$

We generate 5000 data points, which are shown in the histogram in Figure 4.44-(a). The mean (-0.0067) does not differ from zero in a statistically significant way (Ztest pvalue $=60 \%$ ). From the data we determine $\sigma_{\{\text {pop }, \ell\}}^{2}=0.708$.

As will be discussed next, the value of $L_{\mathrm{dom}}^{\prime}$ as determined by the linear regression of the domain-size data is $L_{\text {dom }}^{\prime}=0.06296$. Therefore we would estimate the variance of increment slope to be

$$
\frac{L_{\mathrm{dom}}^{\prime}}{\ell} \sigma_{\{\mathrm{pop}, \ell\}}^{2}=\frac{0.06296}{1.85} 0.708=0.0241
$$

We find this to be in close agreement (15\% relative error) to the value determined by the regression line, 0.0278 . 


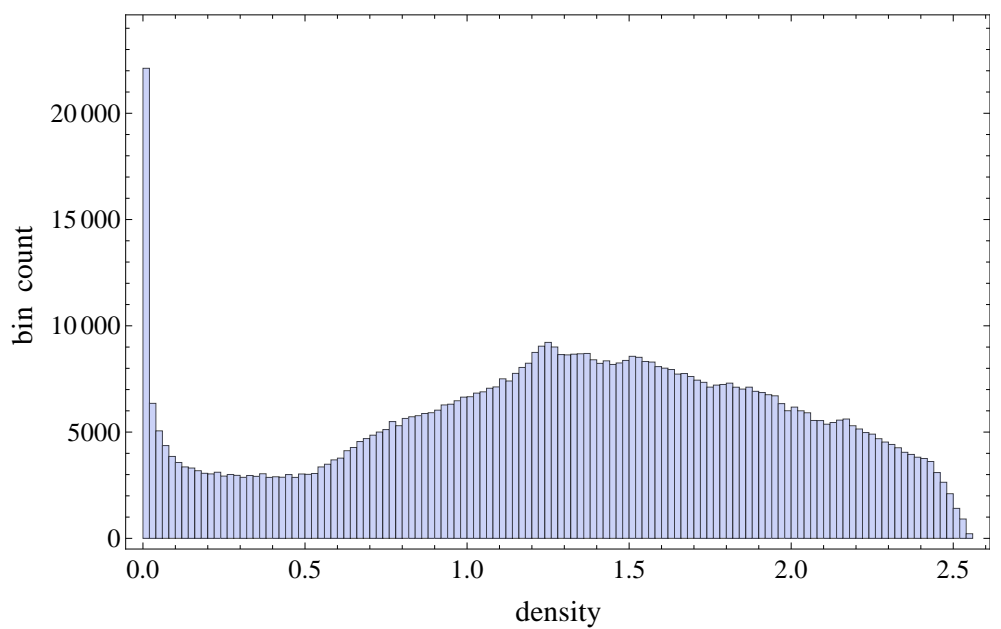

(a) histogram of density values

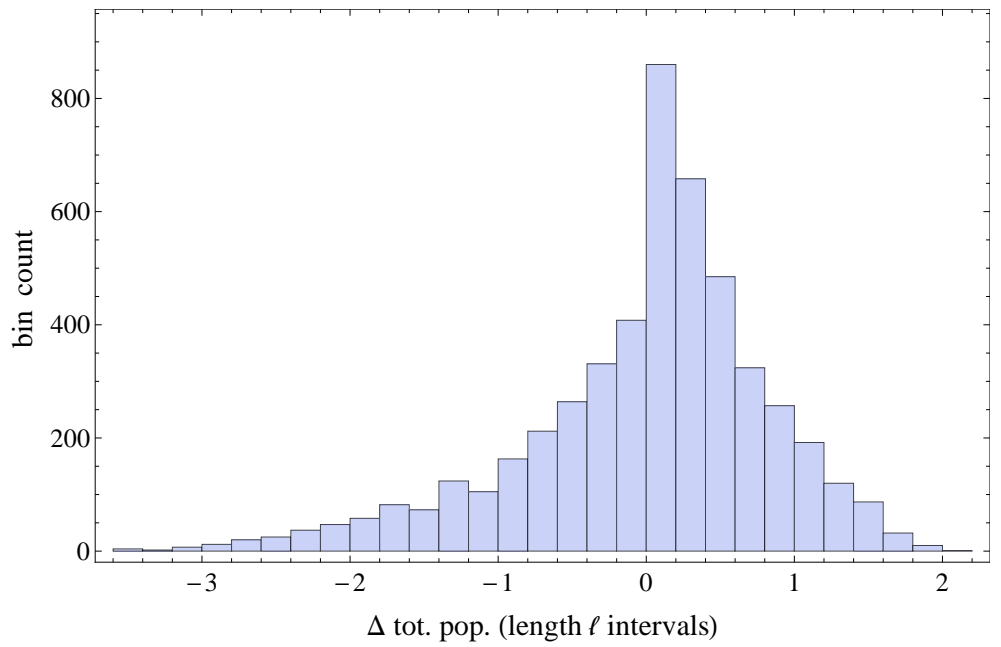

(b) histogram of 1 year tot. pop. changes on intervals of length $\ell=1.85$

Figure 4.44: Sub-figure (a) shows a histogram of all density values on all mesh-points (in populated domain) from $t=900$ to $t=1100$. Sub-figure (b) shows a histogram of the 1 -year total population change on 5000 randomly selected intervals of length $\ell=1.85$, also selected from years $t=900$ to $t=1100$. 


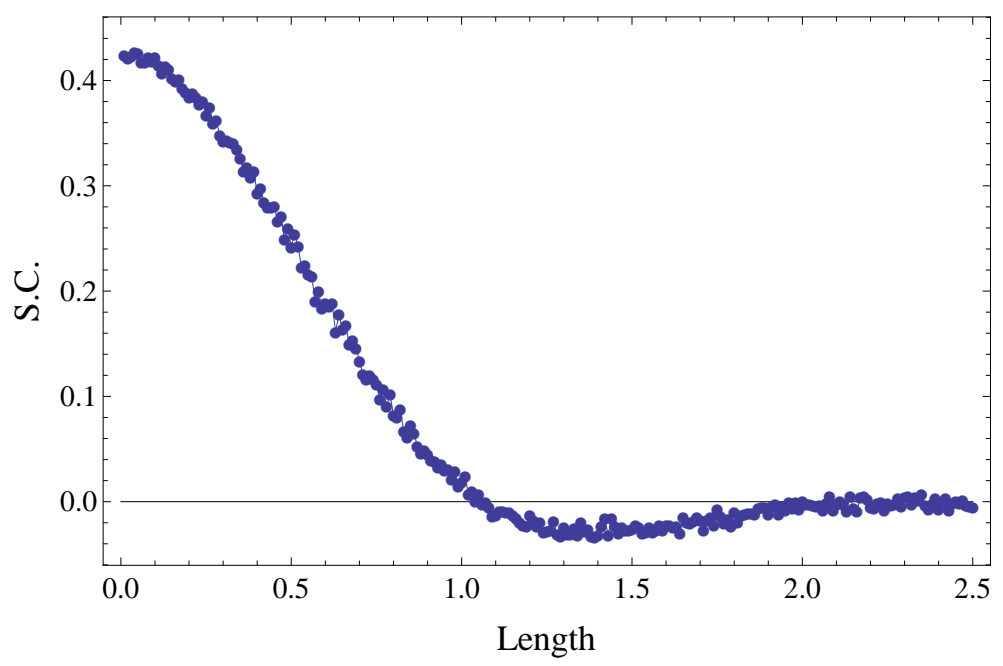

(a) sample covariance vs. length

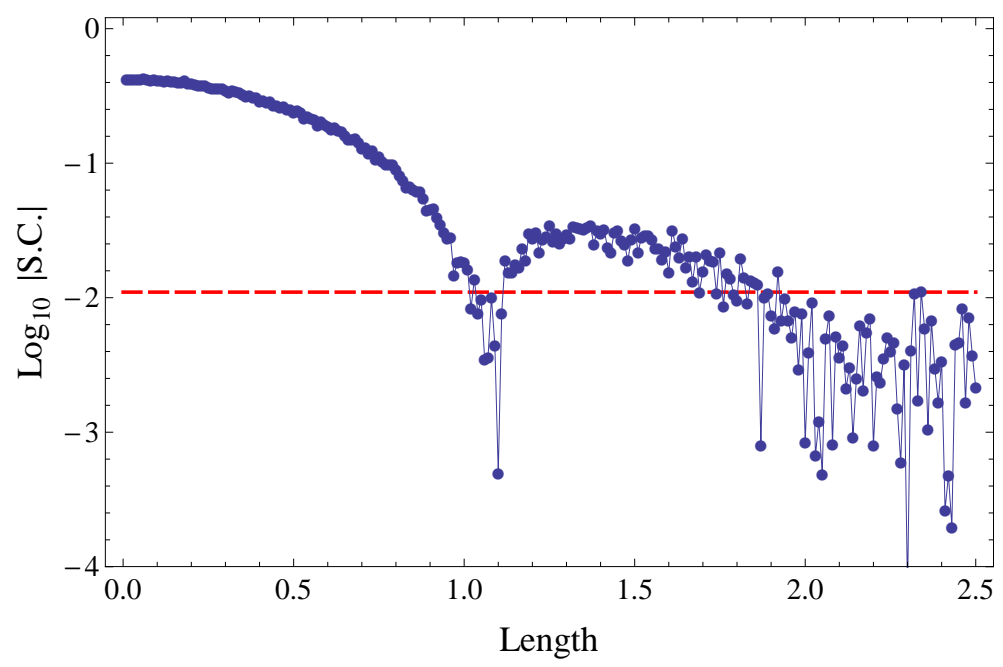

(b) $\log _{10}$ of sample covariance vs. length

Figure 4.45: Sub-figure (a) is plot of the sample covariance of $u_{t}\left(x_{i}\right)$ and $u_{t}\left(x_{i}+s\right)$ for 10,000 points. $t$ is picked randomly from $900, \ldots, 1100$, and $x_{i}$ is picked randomly from the populated domain of $u_{t} . s$ is the value plotted on the x-axis. Sub-figure (b) is $\log _{10}(\mid S . C$. $\mid)$ vs. length. Values falling below the red dashed line can be considered to have no statistically significant covariance.

Next we examine the domain length (denoted as $\operatorname{dom}(t)$ ) versus time relationship. In Figure 4.46-(a) we see the domain length data is well approximated by its linear 
regression. The determined regression equation is

$$
\overline{\operatorname{dom}}(t)=4.634+0.06296 t .
$$

In Figure 4.46-(b) we plot the residual error of the data values and regression, defined by

$$
\text { residual error }=\operatorname{dom}(t)-\overline{\operatorname{dom}}(t) \text {. }
$$

From the plot of the residuals we see that there are long runs of increasing increments of small step size punctuated by dramatic drops. The fact that the overall variance of the residuals remains relatively constant, as opposed to fan shaped, indicates that the distribution of domain length changes, $(\Delta \mathrm{dom})$, is largely independent of time, with perhaps some temporally short range time correlations. In Figure 4.47 we see that the majority of the $\Delta$ dom lie in the interval $(-0.05,0.15)$, corresponding to the short increasing increments of Figure 4.46-(b). It should be noted that in Figure 4.46-(b), the discrete values of $\Delta$ dom are a artifact of the mesh size (0.018) used in the simulation. The outlying values of $\Delta$ dom ranging from -0.1 to -3.3 account for only $0.7 \%$ of the data, but have a large effect on the mean of $\Delta \mathrm{dom}$. The mean of $\Delta \mathrm{dom}$ is 0.064 which is very close to the slope of the regression line. If values of $\Delta$ dom less then -0.1 are censored, the mean becomes 0.072 , with the effect of the bottom $0.7 \%$ quantile decreasing the mean by $12 \%$. 


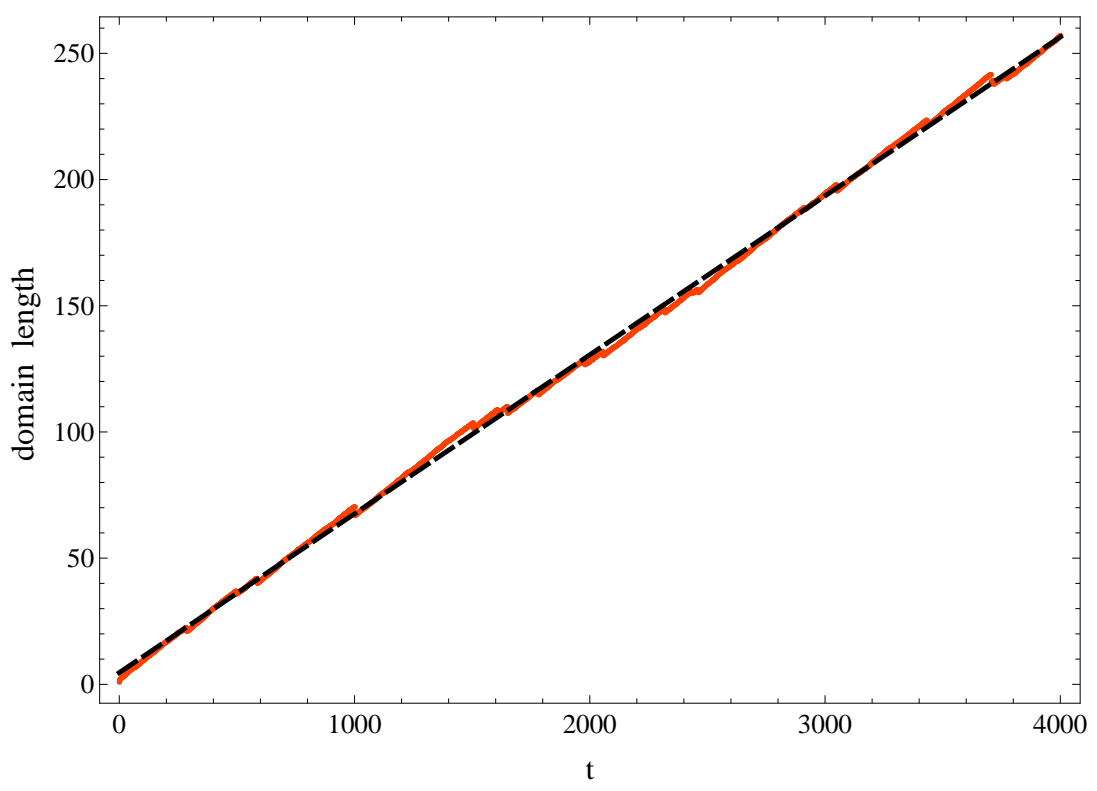

(a) domain length and fitted line vs. time

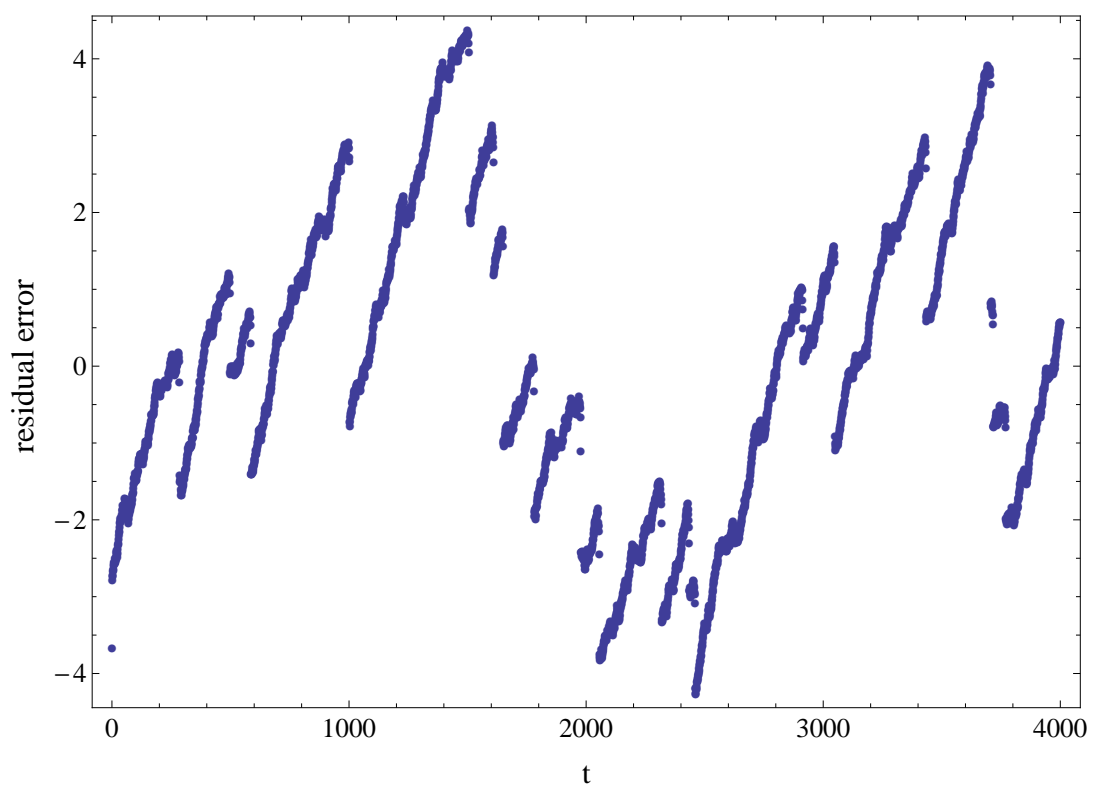

(b) residual error vs. time

Figure 4.46: Sub-figure (a) shows the domain length data (red), and fitted line (dashed black). Sub-figure (b) shows the residual errors vs. time, the residual error is the difference between the data and fitted line. 


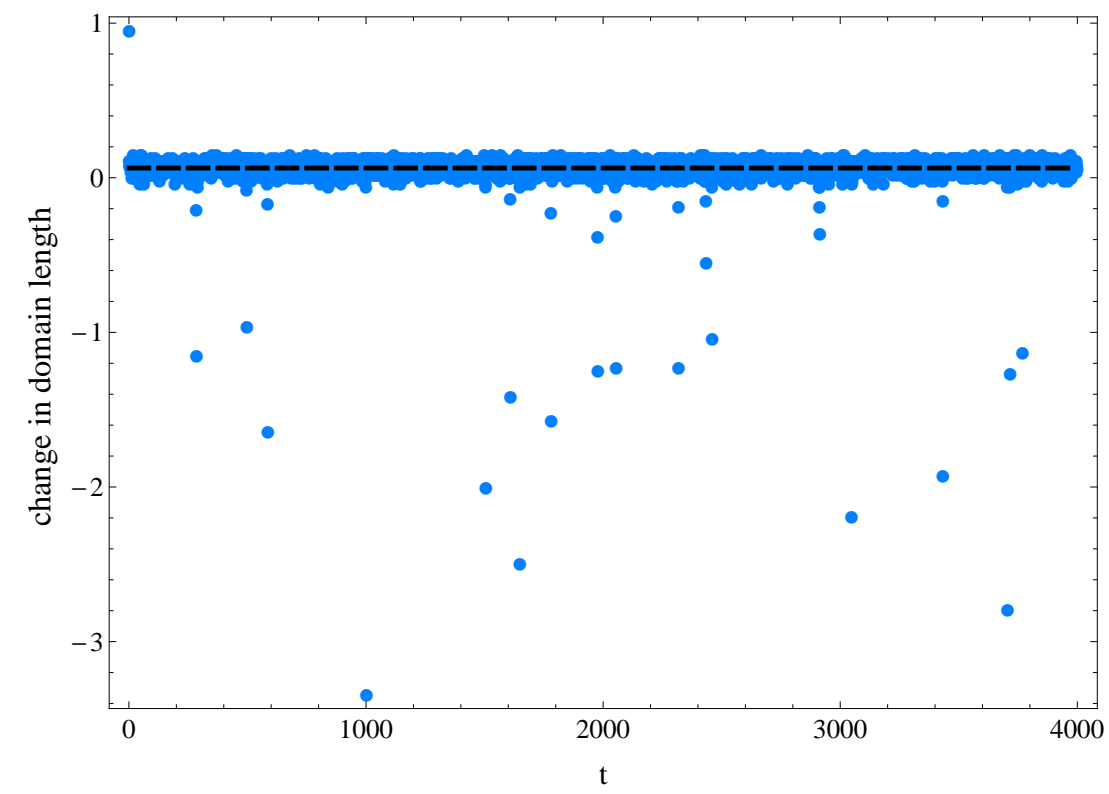

(a) yearly changes in domain length

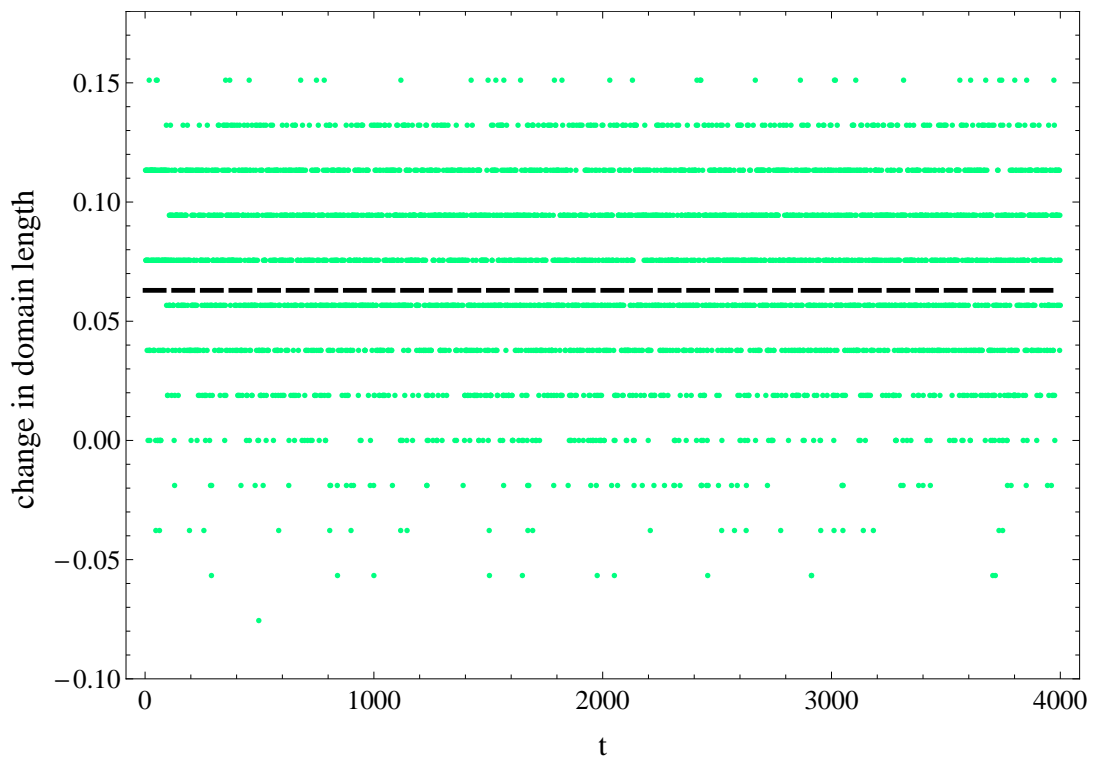

(b) detailed view of domain length changes near $\Delta$ dom $=0$

Figure 4.47: Yearly changes in domain length plotted vs. time. Sub-figure (b) shows a detailed view of $\Delta$ dom near the $\Delta$ dom $=0$ line. The black dashed line is the average value of $\Delta$ dom.

To better understand these large outlying decrements in domain size, we refer to Figure 4.46, where we see a large decrement occurs at approximately $t=1000$. In Figure 
4.48 we show the leftward edge of the solutions from $t=991$ to $t=1002$. In frame $t=997$ we see a large region from $-38<x<-31$ has built up to almost the maximum value of $g(u)$. Due to severe overcompensation we see an isolated population is "calved" from the domain, as can be seen on the frame $t=1000,-38<x<-36$. This population is sub-Allee threshold and subsequently perishes. The overall result being that over the course of $t=991$ to $t=1002$ the leftward edge of the domain decreases in absolute value from $x=-38$ to $x=34$. For reference purposes, Figure 4.49 shows the entirety of $u_{991}(x)$.

Again, referring to Figure 4.46, we see there is a long run of small mostly positive increments of $\Delta$ dom from $t \approx 600$ to $t \approx 1000$. In Figure 4.50 we show the evolution of the rightward edge of the domain from $t=621$ to $t=654$. 


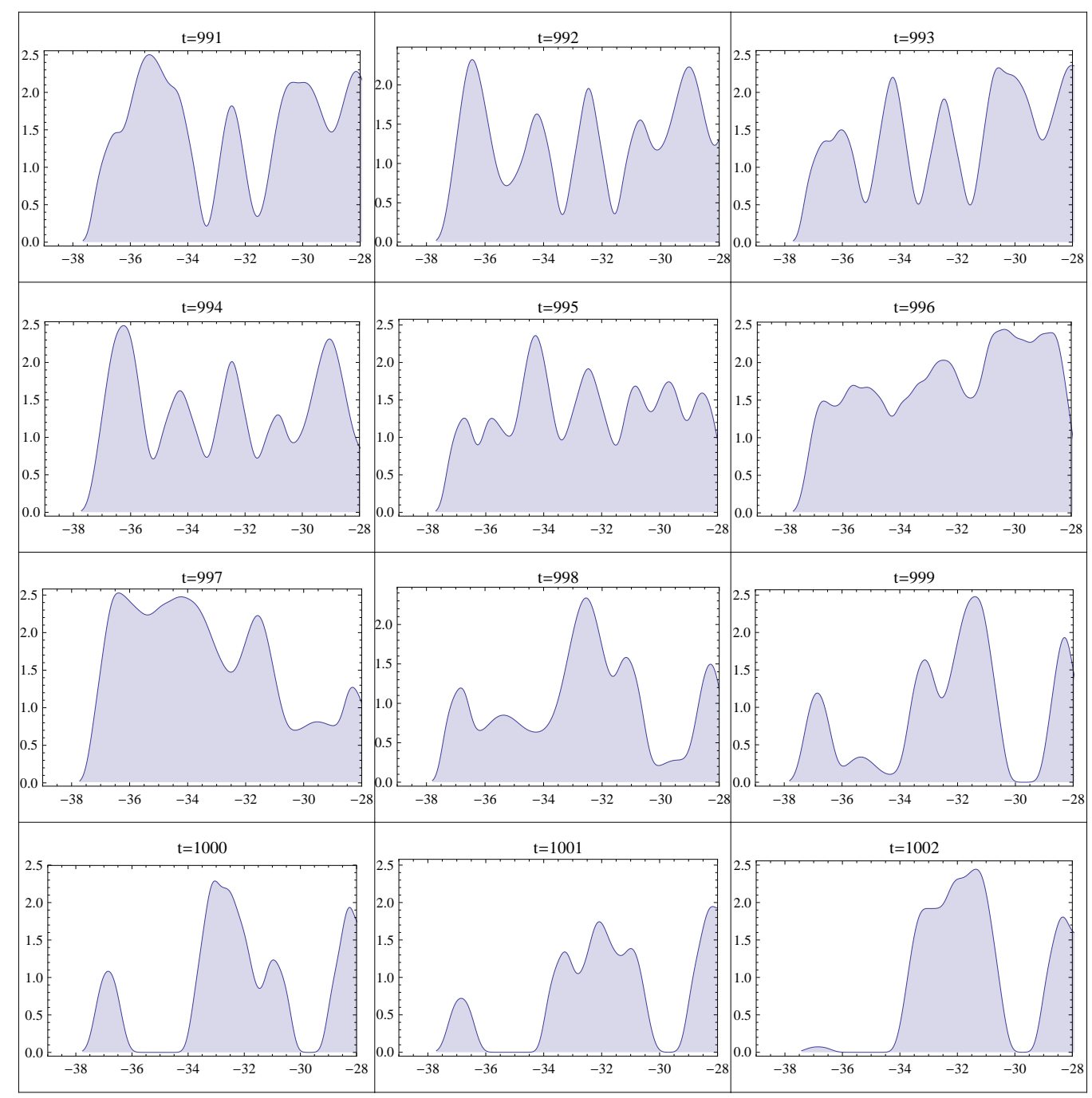

Figure 4.48: An illustration of the calving process 


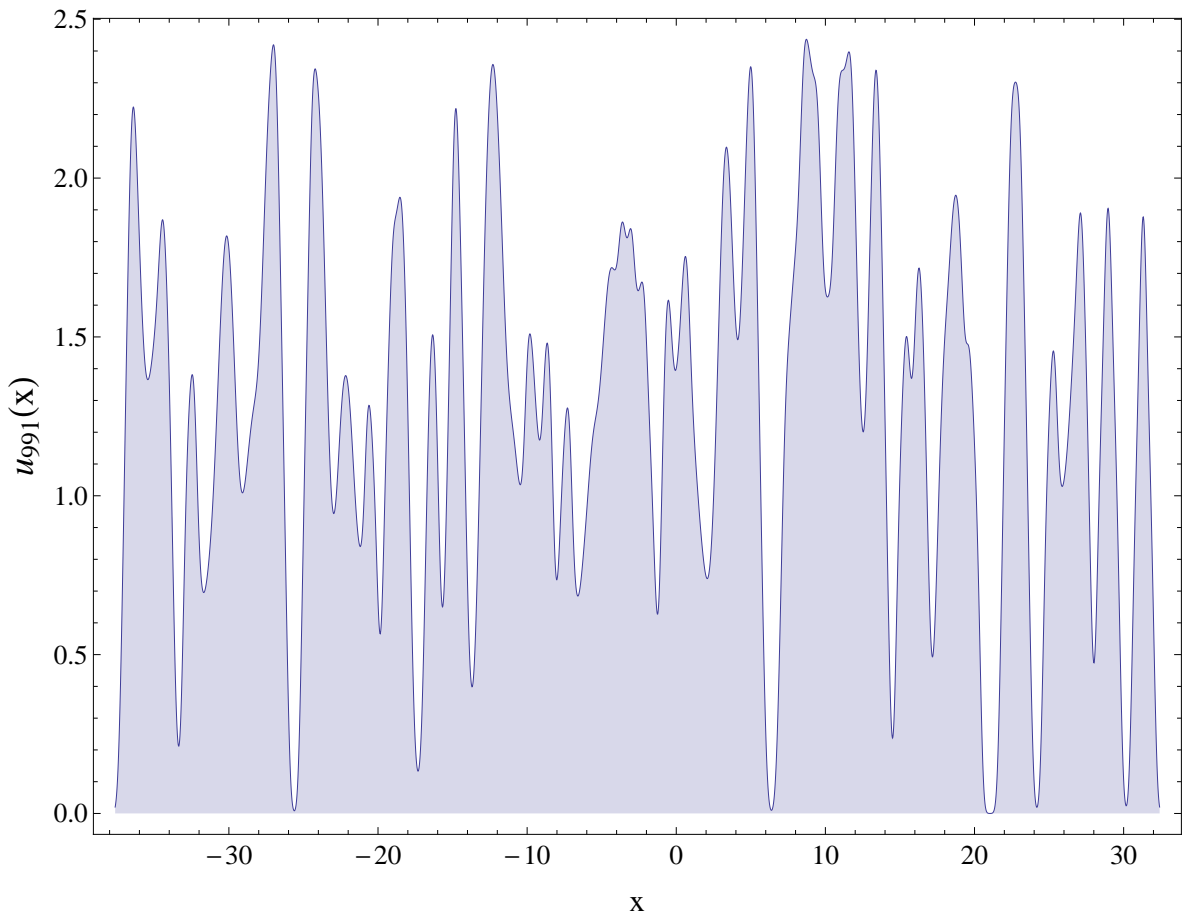

Figure 4.49: A detailed view of $u_{991}(x)$. 


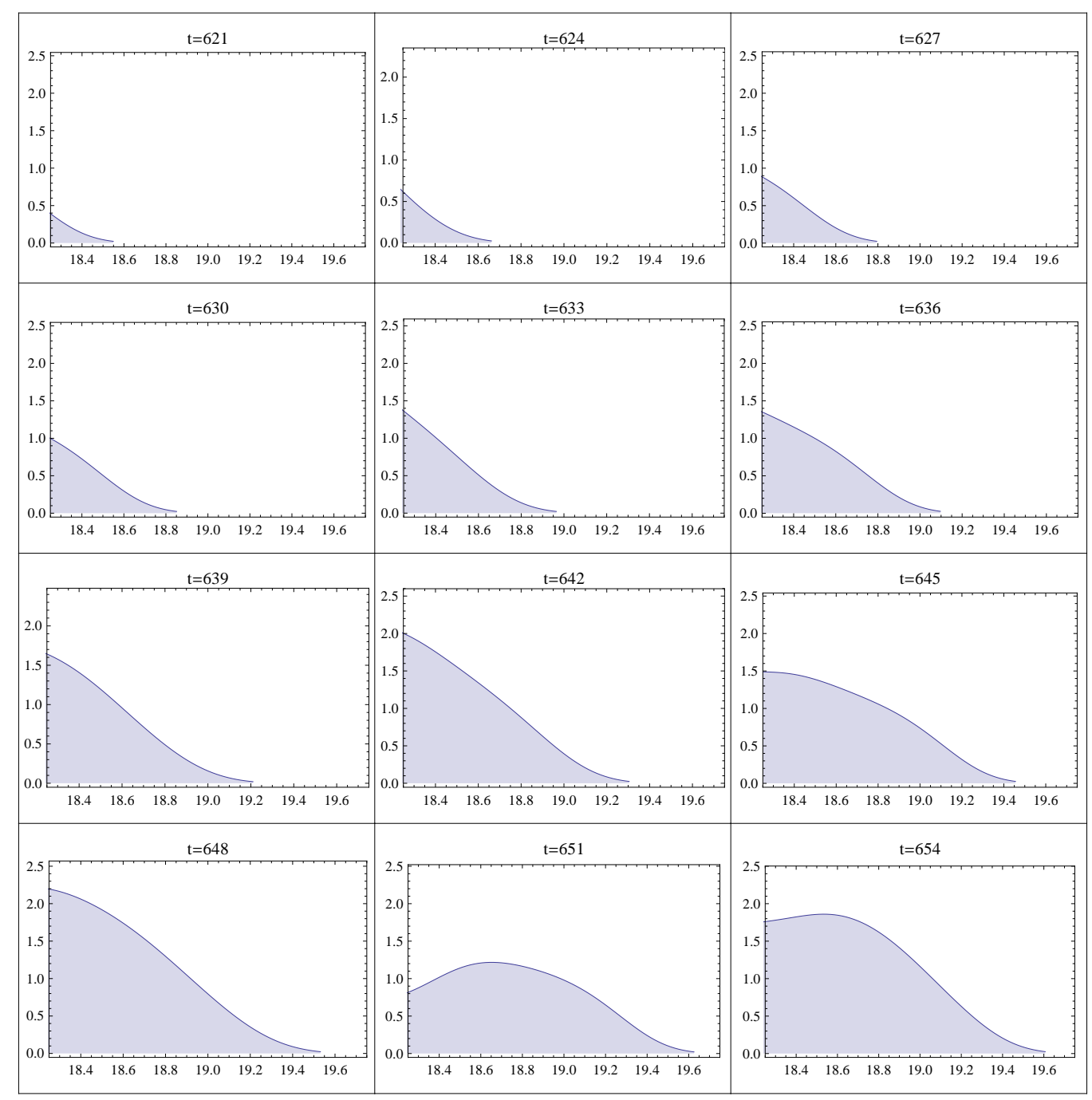

Figure 4.50: An illustration of a run of gradual increases. 


\section{CHAPTER 5 \\ REMARKS AND CONCLUSION}

In this paper we were able to show the rather surprising result that when Allee effect and over-compensation are combined, it is possible to produce solutions that persist only in a bounded domain, even though the environment is completely homogenous. In Chapter 3 we analytically demonstrated the existence of these solutions for the case of our specially chosen 3-parameter piecewise constant growth function. Through the use of the power exponential distribution, we were able to demonstrate that the formation of these solutions favored platykurtic distribution, in the sense of parameter space containment. This relation was illustrated in Figures 3.15-3.17.

In Chapters 4.0.4-4.0.6 we showed that a wide variety of behaviors could emerge when the equilibrium solutions from Chapter 3 are perturbed. Everything from simple period-2 oscillations (e.g. Fig. 4.12), long period oscillations (Fig. 4.5), to apparently chaotic oscillations (fig. 4.14) were observed. So long as the oscillations remain unimodal, the density $u_{t}(x)$ can be described as the convolution of $k$ with $\widetilde{G}_{\left\{-b_{1}(t),-a_{1}(t), a_{2}(t), b_{2}(t)\right\}}$ from Eq. 4.1. Thus the system is actually equivalent to a 4-dimensional discrete time recursion map on the parameters $\left(b_{1}(t), a_{1}(t), a_{2}(t), b_{2}(t)\right)$. In future work we could take advantage of finite-dimensional techniques to better characterize the oscillations of the system.

In Chapter 4.0.4.2 we studied the bifurcation of solutions with the uniform-kernel as a function of the width of the initial data. In the simplest case, such as Fig. 4.6, we find that for narrow initial data, the solution collapses to extinction, for intermediate widths the solution is attracted to a non-spreading periodic orbit, and for wide initial data, the 
solution spreads. In more complicated cases such Fig. 4.9 we see the intermediate values of initial width are punctuated by regions were spreading emerges.

In Chapter 4.07 we numerically demonstrated the existence of either stable or period-2 non-spreading solutions with various realistic growth functions. Clearly there is a need to develop easily testable necessary and/or sufficient conditions on growth functions, kernel pairs to determine when non-spreading conditions occur. One possible route to this goal would be to follow the example in Kot and Schaffer (1986). In the case of determining an equilibrium for a bounded domain problem, Kot converted the IDE into an ODE, and applied standard ODE techniques.

In Chapter 4.0.8.1 we studied the evolution of 2 interacting stable equilibrium as a function of separation distance when the system is initialized with

$$
u_{0}(x)=u_{\mathrm{eq}}\left(x+\frac{w}{2}\right)+u_{\mathrm{eq}}\left(x-\frac{w}{2}\right)
$$

In Fig. 4.24 we find for small separations extinction occurs due to high initial densities facing severe over-compensation. For large separations the two patches persist as the interaction is very weak. For intermediate separations we see merger into a single patch progressively punctuated with extinction. Analogous results are seen for interacting period-2 patches in Chapter 4.0.8.2.

In Chapter 4.0.8.3 and 4.0.8.4 we examine patch emergence from perturbed constant initial data over a large domain. One possible avenue of biological exploration of this phenomena would be that these non-spreading patches could act as population reservoirs during good year/bad year scenarios. For instance, one can imagine a scenario where each year either the "bad year" growth function with non-spreading dynamics $\left(g_{\text {n.sprd }}(u)\right)$ would be chosen with probability $p$, or the "good year" growth function with spreading dynamics $\left(g_{\text {sprd }}(u)\right)$ would be chosen with probability $1-p$. Alternatively, deterministic periodic sequences of $g_{\text {n.sprd }}$ and $g_{\text {sprd }}$ could also be considered. Persistence and spread under such a scenario could be analyzed following the ideas of Nuebert, Kot 
and Lewis (2000).

Finally in Chapter 4.0.9 we demonstrated that a growth function with essential extinction, and a Schwartzain derivative meeting the hypothesis of Schreiber (2003) can undergo spatial spread and linear population growth when coupled to spatial spread. This is in sharp contrast to what is predicted by the non-spatial model, which would be extinction. We examine some statistical properties of the domain and population changes. We offer a statistical explanation as to why the variation in total population increases with domain size. We show that the changes in domain length are mostly small increments, with a small number of outlaying large decrements due to "calving" events as depicted in Fig. 4.48. Clearly there is a great deal of future work required to have a more complete understanding of spread with growth functions exhibiting essential-extinction. 


\section{REFERENCES}

[1] Berestycki H., (2009), Can a species keep pace with a shifting climate? Bulletin of mathematical biology, 71(2), 399-429.

[2] Bertsch M., Hilhorst D., (1986), A density dependent diffusion equation in population dynamics: stabilization to equilibrium, SIAM J. Math. Analysis, 17(4), 863883.

[3] Beverton R.J.H., Holt S.J., (1957), On the dynamics of exploited fish populations, Fisheries Investigations, Series 2, vol. 19, H.M. Stationary Office, London.

[4] Boukal D., Berec L., (2002), Single-species models of the Allee effect: extinction boundaries, sex ratio, and mate encounters, Jour. Math. Biol., 218, 375-394.

[5] Calabrese J.M., Fagan W.F., (2004), Lost in time, lonely, and single: reproductive asynchrony and the allee effect, The American Naturalist, 187(2), 151-166.

[6] Casella G., Berger R., (2002), Statistical Inference, 2e, Cengage Learning, Boston MA

[7] Deng J., et al., (2012), Modela and tests of optimal density and maximal yeild for crop plants, PNAS, 109(39), 15823-15828.

[8] de Roos A, McCawley E, Wilson W, (1998), Pattern formation and the spatial scale of interactions between predators and prey, Theo. Pop. Biol. ,53, 108-130.

[9] Edelstein-Keshet L., Mathematical models in biology, Philadelphia PA, SIAM. 
[10] El-Sayed A.M.A., et al., (2009), Exact solutions of fractional order biological population models, Commum. Theor. Phys., 52, 992.

[11] Escola H., Geritz S., (2007), On the mechanistic derivation of various discrete-time population models, Bull. Math. Biol., 69, 329-346.

[12] Fagan W., et al., (2005), When can herbivores slow or reverse the spread of an invading plant? A test case from Mount St. Helens, American Naturalist, 166, 669685.

[13] Fitt B., et al., (1987), Spore dispersal and plant disease gradients; a comparison between two emperical models, J. of Phytopathology, 118, 227-242.

[14] Fisher R.A., (1937), The wave of advance of advantageous genes, Ann. Eugenics 7:353-369.

[15] Grinrod P., (1996), The Theory and Applications of Reaction-Diffusion Equations, Oxford, Clarendon Press.

[16] Groom MJ, (1998), Allee effects limit population viability of an annual plant, American Naturalist, 151, 487-496.

[17] Gyllenburg M., Osipov A.V., Söderbacka G., (1996), Bifurcation analysis of a metapopulation model with sources and sinks, J. Nonlinear Sci., 6, 329-366.

[18] Hopf F.A., Hopf F.W., (1985), The role of the Allee effect in species packing, Theor. Pop. Biol., 27, 27-50.

[19] Huang J., Shen W., (2009), Speeds of spread and propagation of KPP models in a time almost and space periodic media, SIAM J. Appl. Dyn. Syst., 790-821.

[20] Jones C.G., Lawton J.H., Shachak M., (1997), Positive and negative effects of organisms as physical ecosystem engineers, Ecology, 78, 1946-1957. 
[21] Kolmogorav A., Petrovskii I., Piscounov N., (1937), Etude de lequation de la diffusion avec croissance de la quantile de matriere et son application a un probleme biologique, Moscou Univ. Math. Bull. 1:126

[22] Kot M., Schaffer W.M., (1986), Discrete-time growth-dispersal models, Mathematical Biosciences, 80(1), 109-136.

[23] Kot M., Lewis M., van den Driessche P., (1996), Dispersal data and the spread of invading organisms, Ecology, 77(7), 2027-2042.

[24] Li B., Lewis M., Weinberger H., (2009), Existence of traveling waves for integral recursions with nonmonotone growth functions, Mathematical Biology, 58, 323338.

[25] Li B., Fagan W.F., Wang C., Otto G., (2014), Spreading speeds and traveling wave solutions in a competitive reaction-diffusion model for species persistance in a stream, Discrete and Cont. Dyn. Sys.-Series B, 19(10), 3267-3281.

[26] Li B., Bewick S., Shang J., Fagan W.F., (2014), Persistance and spread of a species with a shifting habitat edge, SIAM J. Appl. Math, 74, 1397-1417.

[27] Lindstrom J., Kokko H., (1998), Sexual reproduction and population dynamics: the role of polygyny and dempographic sex differences, Proc. of the Royal Society B, 265, 483-488.

[28] Liu R., (1985), A nonlinear integral operator arising from a model in population genetics III, Heterozygote inferior case, SIAM J. Math. Analysis, 16(6), 1180-1211.

[29] Lutscher F, (2008), Density-dependant dispersal in integrodifference equations, J. Math. Biol., 56, 499-524.

[30] Lutscher F., Seo G., (2011), The effect of temporal variability on persistance conditions in rivers, J. Theor. Biol., 283, 53-59. 
[31] Meyer K, Li B, (2013), A spatial model of plants with an age-structured seed bank and juvenile stage, SIAM J. of Applied Math., 73, 1676-1702.

[32] Murray J.D., (2001), Mathematical Biology, Berlin, Springer-Verlag

[33] Neubert M, Kot M, Lewis M, (1995), Dispersal and pattern formation in a discretetime predator-prey model, Theo. Pop. Biol., 48,7-43.

[34] Neubert M, Kot M, Lewis M, (2000), Invasion speeds in fluctuating enviroment, Proc. of Royal Society B, 267, 1603-1610.

[35] Okubo A., et al., (1989) On spatial spread of the grey squirrels in Britain, Proc. of the Royal Society series b-biological sciences, 238(1291), 113-125.

[36] Otto G., Bewick S., Li B., Fagan W.F., (2017), How phenological variation affects species spreading speeds accepted by Bull. Math Biol., copy on file with author.

[37] Owen M.R., Lewis M.A., (2001), How predation can slow, stop, or reverse a prey invasion, Bull. Math. Biol., 63(4), 655-684.

[38] Patapov A.B., Lewis M.A., (2004), Climate and competition: the effect of moving of moving range boundaries on habitat invasibility, Bull. Math. Biol. 66:975-1008.

[39] Schreiber S., (2001), Chaos and sudden extinction in simple ecological models, J. Math. Biol., 199, 407-414.

[40] Schreiber S., (2003), Allee effects, extinctions, and chaotic transients in simple population models, Theoretical Population Biology, 64,201-209.

[41] Shi J., Shivaji R., (2006), Persistance in reaction diffusion models with weak Allee effect, J. Math. Biol. 52, 807-829. 
[42] Seo G., Lutscher F., (2011) Spread rates under temporal variability: calculation and applications to biological invasions, Mathematical Models and Methods in Applied Sciences, 21(12), 2469-2489.

[43] Skellam J.G., (1951), Random dispersal in theoretical populations, Biometrika, 38:196-218.

[44] Stevens P, Sutherland W, (1999), Consequences of the Allee effect for behavior, ecology, and conservation, TREE, 14, 401-405.

[45] Sullivan L., et al, (2017), Density dependence in demography and dispersal generates fluctuating invasion speeds, PNAS, 114(19), 5053-5058.

[46] Tillman P., (1982), Resource competetion and community structure, Monographs in Population Biology, Vol 17, Princeton University Press, Princeton NJ.

[47] van Kirk R., Lewis M., (1997), Integrodifference models for persistance in fragmented habitats, Bull. of Math. Biol., 59(1), 107-137.

[48] Vasilyeva O., Lutscher F., Lewis M., (2016), Analysis of spread and persistance for stream insects with winged adult stages, J. Math. Biol., 72, 851-875.

[49] Veit R, Lewis M, Dispersal, population growth, and the Allee effect: dynamics of the house finch invasion of eastern North America, Amer. Naturalist, 148, 255-274.

[50] Wang M., Kot M., Neubert M., (2002), Integrodifference equations, Allee effects, and invasions, 44, 150-168.

[51] Weinberger H, (1978), Asymptotic behavior of a model in population genetics, in Nonlinear Partial Differential Equations and Applications, J. Chadam, ed., Lecture Notes in Mathematics 648, Springer, New York, pg 47-98. 
[52] Weinberger H, (1982), Long-time behavior of a class of biological models, SIAM J. Math. Analysis, 13, 353-396.

[53] Wilson M., (1992), The ecology of seed dispersal, pages 61-85, in M. Fenner, editor Seeds:the ecology of regenerationin plant communities. CAB International, Wallington, Oxon, UK.

[54] Wilson W, Nisbet R, (1997), Cooperation and competition along smooth enviroment gradients, Ecology, 78, 2004-2017.

[55] Zhou Y, Kot M, (2011), Discrete-time growth-dispersal models with shifting species ranges, Theoretical Ecology, 4, 1325. 


\title{
CURRICULUM VITAE
}

\author{
Garrett Luther Otto
}

\section{$\underline{\text { Academic Record }}$}

University of Louisville, Louisville, $K Y$

Ph.D. in Applied and Industrial Mathematics

Advisor: Dr. Bingtuan Li

Expected December 2017

University of Kentucky, Lexington, $K Y$

B.S. Physics and Mathematics

August 2001

\section{$\underline{\text { Publications }}$}

-Otto, G., Li, B., (2017), Spatially localized equilibrium solutions for integro-difference equations with Allee and over-compensation, (manuscript submitted to Journal of Mathematical Biology).

-Otto, G., Bewick, S., Li, B., et al., (2017), How phenological variation effects species spread speeds, (accepted by Bulletin of Mathematical Biology, Sept. 2017), (copy on file with author).

-Li, B., Fagan, W.F., Otto, G., Wang, C., (2014), Spreading speeds and traveling wave solutions in a competitive reaction-diffusion model for species persistence in a stream, Discrete and Continuous Dynamical Systems Series B, 19, 3267-3281.

\section{Presentations}

\section{Conference Presentations}

- Compact equilibrium solutions for an integro-difference equation with Allee and overcompensation effects, Spring 2017 AMS Central Sectional Meeting, Bloomington IN, April 1, 2017

-Phenologically explicit reaction-diffusion model, Spring South Eastern AMS Sectional Meeting, Huntsville AL, March 29, 2015 


\section{Other Talks}

-A review of Olga Vasilyeva's paper: Analysis of spread and persistence for stream insects with winged adult stages, University of Louisville mathematics graduate seminar. (October 2016)

- Compact equilibrium solutions for an integro-difference equation with Allee and overcompensation effects, University of Louisville mathematical biology seminar. (September 2016)

-Applications of control theory in biology, University of Louisville mathematics graduate seminar. (March 2016)

\section{Teaching Experience}

\section{University of Louisville, Graduate Teaching Assistant 2013-2017}

\section{$\underline{\text { Lead Instructor }}$}

-MA205: Calculus I

Summer 2016

-MA205: Calculus I

Summer 2015

-MA105: Contemporary mathematics, a terminal course dealing with financial math, voting and apportionment theory

Summer 2013

\section{Teaching Assistant}

-MA111: College Algebra

Spring 2016

-MA105: MA109: Elementary Statistics

Fall 2015

-MA180: Business Calculus

Spring 2015

-MA105: MA180

Fall 2014

-MA111: College Algebra

\section{Programming Languages}

- Mathematica

- MatLab

- $\mathrm{R}$

- LATEX 